Florida International University FIU Digital Commons

\title{
Intracranial Volume Estimation and Graph Theoretical Analysis of Brain Functional Connectivity Networks
}

Saman Sargolzaei

Florida International University, ssarg004@fiu.edu

DOI: $10.25148 /$ etd.FI15032115

Follow this and additional works at: https://digitalcommons.fiu.edu/etd

Part of the Biomedical Commons, and the Neuroscience and Neurobiology Commons

\section{Recommended Citation}

Sargolzaei, Saman, "Intracranial Volume Estimation and Graph Theoretical Analysis of Brain Functional Connectivity Networks" (2015). FIU Electronic Theses and Dissertations. 1915.

https://digitalcommons.fiu.edu/etd/1915 


\section{FLORIDA INTERNATIONAL UNIVERSITY}

Miami, Florida

INTRACRANIAL VOLUME ESTIMATION AND GRAPH THEORETICAL ANALYSIS OF BRAIN FUNCTIONAL CONNECTIVITY NETWORKS

A dissertation submitted in partial fulfillment of the

requirements for the degree of

DOCTOR OF PHILOSOPHY

in

ELECTRICAL ENGINEERING

by

Saman Sargolzaei 
To: Dean Amir Mirmiran

College of Engineering and Computing

This dissertation, written by Saman Sargolzaei, and entitled Intracranial Volume Estimation and Graph Theoretical Analysis of Brain Functional Connectivity Networks, having been approved in respect to style and intellectual content, is referred to you for judgment.

We have read this dissertation and recommend that it be approved.

Armando Barreto

Mercedes Cabrerizo

Sakhrat Khizroev

Naphtali David Rishe

Malek Adjouadi, Major Professor

Date of Defense: March 25, 2015

The dissertation of Saman Sargolzaei is approved.

$\begin{array}{r}\text { Dean Amir Mirmiran } \\ \text { College of Engineering and Computing } \\ \hline \begin{array}{r}\text { Dean Lakshmi N. Reddi } \\ \text { University Graduate School }\end{array}\end{array}$

Florida international University, 2015 
CC Copyright 2015 by Saman Sargolzaei

All rights reserved. 


\section{DEDICATION}

To my dear parents

and

Shirin \& Arman

the blessings of my life 


\section{ACKNOWLEDGMENTS}

First and foremost, I would like to express my sincerest gratitude to my major professor and the director of Center for Advanced Technology and Education (CATE), Dr. Malek Adjouadi. I can hardly forget the amount of happiness and motivation I have experienced since I met Dr. Adjouadi for the first time and expressed my interests in studying Ph.D. under his supervisory. Today, I acknowledge that I can never thank him enough for his constant support, guidance, patience and expertise. Without a doubt, he is a true inspiration in my career life. My thanks goes to my co-major professor, Dr. Mercedes Cabrerizo, for her support on many technical and clinical issues related to understanding electroencephalography (EEG) data and in developing appropriate algorithms for analyzing and processing EEG data.

I would also like to thank my committee members Dr. Armando Barreto, Dr. Sakhrat Khizroev and Dr. Naphtali David Rishe for their valuable input and support. I wish to extend my warmest regards to Dr. Jean Andrian for his kind support and guidance. Many thanks also goes to Ms. Pat Brammer from the ECE department.

I am indebted to my previous mentors and advisers, Dr. Karim Faez, Dr. Hamid Hassanpour, Dr. Ishwar K. Sethi, Dr. Nilesh Patel, the Dr. Akmal Younis, Dr. Fatta Nahab and Dr. Mohammed Abdel-Mottaleb, for their support over the years. I also would like to appreciate my colleagues at CATE, especially Dr. Anas Salah Eddin, whose discussions and inputs was insightful in my research career.

I would like to thank many families and friends who have supported me in many ways during the course of my Ph.D., especially Mr. Noei’s family, Maria and Jesus, Ms. Sahifi, 
Mr. Ebrahimi's family, Mr. Salemi's family, Behnam Sargolzaei, Shahab Sargolzaei, Mohammad Hadi Amini, Ali Ziaei and Mahdi Jamei. My thanks also go to Ms. Niovi Rojas for the help she provided me as manager of the CATE Center.

I wish to recognize the support provided by the National Science Foundation under grants CNS-0959985, CNS-1042341, HRD-0833093, and IIP-1230661, as well as the Ware Foundation for their generous support. I also acknowledge Donors of Charles E. Perry Graduate Scholarship, Engineering Dean's Merit and Leadership Scholarship, SGA Graduate Scholarship, which facilitated the research of the current dissertation. 


\begin{abstract}
OF THE DISSERTATION
INTRACRANIAL VOLUME ESTIMATION AND GRAPH THEORETICAL ANALYSIS OF BRAIN FUNCTINAL CONNECTIVITY NETWORKS
\end{abstract}

\author{
by
}

Saman Sargolzaei

Florida International University, 2015

Miami, Florida

\title{
Professor: Malek Adjouadi, Major Professor
}

Understanding pathways of neurological disorders requires extensive research on both functional and structural characteristics of the brain. This dissertation introduced two interrelated research endeavors, describing (1) a novel integrated approach for constructing functional connectivity networks (FCNs) of brain using non-invasive scalp EEG recordings; and (2) a decision aid for estimating intracranial volume (ICV). The approach in (1) was developed to study the alterations of networks in patients with pediatric epilepsy. Results demonstrated the existence of statistically significant $(\mathrm{p}<0.01)$ difference in the FCNs of the pediatric patients with epilepsy and control patients. Two novel decision support systems (GLM-based and GMM-based) for epilepsy diagnosis were developed and evaluated using resting state scalp EEG of 18 subjects ( 7 controls and 11 epilepsy subjects). The GMM-based system resulted in accuracy of $88.8 \%$ with $81.8 \%$ sensitivity and $100 \%$ specificity in diagnosing epilepsy without a priori knowledge. Analysis of network alterations during interictal recordings of patients with severe form of epilepsy showed significant alteration of the global efficiency of the networks during interictal period. Decision aid was established for defining protocols for 
ICV estimation across different subject groups in terms of (1) sampling frequencies, and (2) type of software. A group of 53 subjects were considered, including adult controls (AC), adults with Alzheimer's disease (AD), pediatric controls (PC) and pediatric epilepsy subjects (PE). Reference measurements were calculated for each subject by manually tracing intracranial cavity without sub-sampling. The reliability of reference measurements were assured through intra- and inter- variation analyses. Three publicly well-known software packages (Freesurfer, FSL, SPM) were examined. Results with a $95 \%$ confidence showed that in order to keep the accuracy of the inter-leaved slice sampling protocol above 99\%, sampling period cannot exceed 20 millimeters for AC, 25 millimeters for PC, 15 millimeters for AD and 17 millimeters for the PE groups. Tuning of the parameters in FSL and the use of proper atlas in SPM showed significant reduction in the systematic bias and the error in ICV estimation. 


\section{TABLE OF CONTENTS}

CHAPTER

PAGE

1

Introduction

General Statement of Problem Area........................... 1

1.3

Research Purpose... 3

1.4

Significance of the Study. ... 3

1.5

Research Questions and Hypotheses............................ 4

Structure of the Research..................................... 5

$2 \quad$ Literature Review and Design Fundamentals ......................... 8

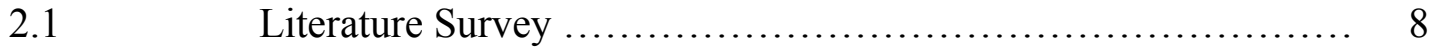

2.1.1

2.1.2

2.2

2.3

2.4

2.5

2.6

Brain Functional Connectivity Networks ................ 8

Intracranial Volume Estimation ............................ 12

Scalp Electroencephalography .............................. 15

Magnetic Resonance Imaging ................................. 16

General Linear Model .......................................... 17

Gaussian Mixture Model ........................................ 18

Graph Theory and Network Measures .......................... 19

3. Brain Functional Connectivity Network …......................... 22

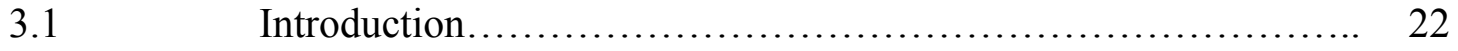

3.2

3.2 .1

3.2 .2

3.2 .3

3.2 .4

3.2 .5

3.2 .6

3.3

3.3.1

3.3.2

3.3.3

3.3.4

3.3 .5

3.4

Algorithm I: GLM-based Approach............................. 22

Data Acquisition....................................... 22

Preprocessing..................................... 23

Functional Connectivity Network Construction........... 23

Graph Analysis of FCN's.............................. 27

Decision Making and GLM-based Feature Selection........ 28

Implementation Results.............................. 31

Algorithm II: GMM-based Approach......................... 37

Subjects and Data................................... 37

Functional Connectivity Network Construction........... 39

Graph Mapping of Brain FCN's and Feature Extraction... $\quad 40$

GMM-based Decision Making Process.................... 42

Implementation Results.................................. 44

Conclusion............................................... 51

4. Temporal Analysis of FCNs in Epilepsy............................. 52

$4.1 \quad$ Introduction...................................................... 52

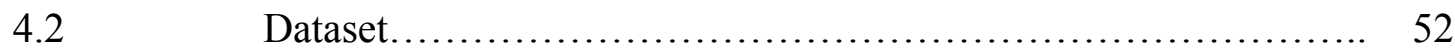

$4.3 \quad$ Methods..................................................... 53

4.3.1 Ictal Events Segmentation............................ 53

4.3.2 Functional Connectivity Networks Construction.......... 53

4.3.3 Brain FCN's Binarization............................. 54

4.3.4 Graph Theory Analysis of FCN's....................... 54 

Group Analysis..................................... 82

5. Intracranial Volume Estimation.................................... 89

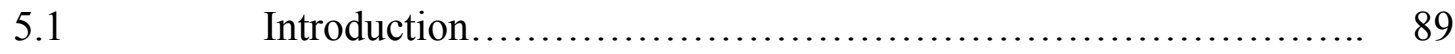

5.2 Materials and Methods...................................... 89

5.2.1 Subjects and Images............................... 91

5.2.2 Reference ICV Estimation........................... 92

5.2.3 Automatic ICV Estimation............................ 94

5.2.4 Reliability Assessment of Manual ICV Estimation........ 95

5.2.5 Statistical Analysis of Sampling Based Technique........ 95

5.2.6 Reliability Assessment of Automatic ICV Estimation...... 96

$5.3 \quad$ Results............................................... 97

$5.4 \quad$ Conclusion................................................. 105

6. Conclusions and Recommendations for Future Work.................. 108

$6.1 \quad$ Conclusions................................................. 108

6.2 Discussions................................................ 109

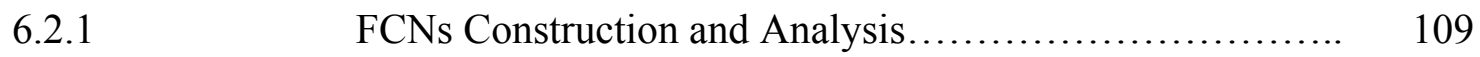

6.2.2 Intracranial Volume Estimation.......................... 109

Recommendations for Future Work.......................... 118

References....................................................... 121

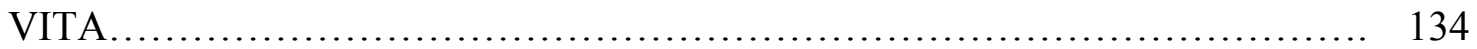




\section{LIST OF TABLES}

TABLE

3.1 Demographic data of study subjects............................. 24

3.2 List of topological features to extract from graph corresponding to FCN... 28

3.3 First and second moment statistics for feature vectors across group of subjects.

3.4 Clustering Results with no prior knowledge on diagnosis............... 36

3.5 Demographic characteristics of subjects.......................... 38

3.6 Graph theoretical features of functional connectivity networks........... 41

3.7 First and second statistics plus statistical analysis of feature vectors....... 47

3.8 Connectivity strength for left hemisphere, right hemisphere and interhemisphere...

3.9 Clustering results with no prior knowledge provided on diagnosis........ 50

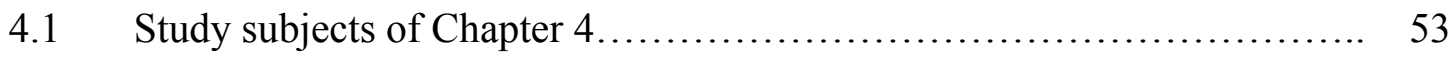

4.2 Two-tailed paired t-test results ( $p$ values) of the means of each network measure across group for different stages of the evolution of interictal recordings

5.1 Demographic characteristics of study subjects

5.2 Intra- and inter- operator variation analysis for manual ICV estimation in different subject groups

5.3 Software reliability analysis for automated ICV estimation versus reference estimation $(\mathrm{Op} 1)$.

5.4 Intra- software (phase I and phase II) variation analysis for automated ICV estimation................................................ 104

6.1 Main factors analysis and their interactions on the measured ICV ............................................................ 
6.2 Different versions of SPM software analysis........................... 116

6.3 The Effect of Parameter self-tuning in using FSL for ICV estimation..... 117 


\section{LIST OF FIGURES}

FIGURE

2.1 International 10-20 electrode placement system. F: Frontal; C: Central; P: Parietal; T: Temporal; O: Occipital. Odd numbers correspond to left hemisphere and even numbers correspond to right hemisphere............ 16

3.1 Flowchart of the suggested decision support system to differentiate pediatric with epilepsy from pediatric control.

3.2 Correlation matrix (Left), Average correlation coefficient for each feature with other features (Right)

3.3 Visualization of constructed undirected functional connectivity networks.. 32

3.4 Graph representations of average of constructed FCNs over the pediatric control (PC) group and pediatric epilepsy (PE) group. Thickness of links (graph edges) shows the strength of connectivity among electrode pairs...

3.5 Statistical significance of the group difference for network graph measures as the function of threshold

3.6 Flowchart of the decision support system........................... 37

3.7 Flowchart of the two phase decision making process. ................. 43

3.8 Visualization of constructed undirected FCNs $\ldots \ldots \ldots \ldots \ldots \ldots \ldots \ldots \ldots, 46$

3.9 Graph representations of average of constructed FCNs................ 48

4.1 Functional connectivity networks corresponding to pre- inter- post- ictal

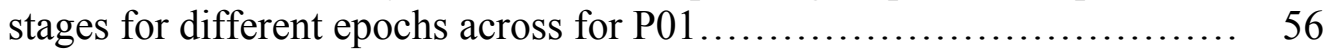

4.2 Graph Theory Measurements corresponding to pre- inter- post- ictal stages

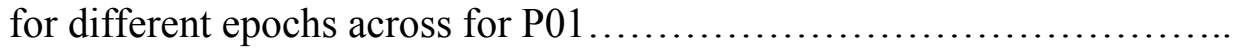

4.3 Functional connectivity networks corresponding to pre- inter- post- ictal stages for different epochs across for P02 ....................... 58

4.4 Graph Theory Measurements corresponding to pre- inter- post- ictal stages

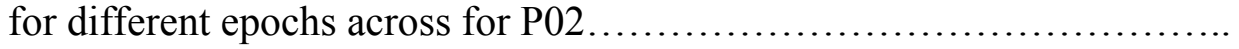

4.5 Functional connectivity networks corresponding to pre- inter- post- ictal 
stages for different epochs across for P03.

4.6 Graph Theory Measurements corresponding to pre- inter- post- ictal stages for different epochs across for P03

4.7 Functional connectivity networks corresponding to pre- inter- post- ictal stages for different epochs across for P04....

4.8 Graph Theory Measurements corresponding to pre- inter- post- ictal stages

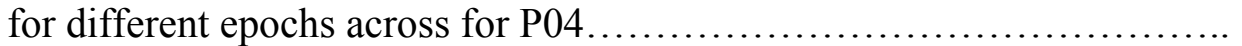

4.9 Functional connectivity networks corresponding to pre- inter- post- ictal stages for different epochs across for P05.

4.10 Graph Theory Measurements corresponding to pre- inter- post- ictal stages for different epochs across for P05.

4.11 Functional connectivity networks corresponding to pre- inter- post- ictal stages for different epochs across for P06....

4.12 Graph Theory Measurements corresponding to pre- inter- post- ictal stages for different epochs across for P06.

4.13 Functional connectivity networks corresponding to pre- inter- post- ictal stages for different epochs across for P07

4.14 Graph Theory Measurements corresponding to pre- inter- post- ictal stages for different epochs across for P07.

4.15 Functional connectivity networks corresponding to pre- inter- post- ictal stages for different epochs across for P08...

4.16 Graph Theory Measurements corresponding to pre- inter- post- ictal stages for different epochs across for P08.

4.17 Functional connectivity networks corresponding to pre- inter- post- ictal stages for different epochs across for P09.

4.18 Graph Theory Measurements corresponding to pre- inter- post- ictal stages for different epochs across for P09.

4.19 Functional connectivity networks corresponding to pre- inter- post- ictal stages for different epochs across for P10...........................

4.20 Graph Theory Measurements corresponding to pre- inter- post- ictal stages 
for different epochs across for P10.

4.21 Functional connectivity networks corresponding to pre- inter- post- ictal stages for different epochs across for P11 ...........................

4.22 Graph Theory Measurements corresponding to pre- inter- post- ictal stages for different epochs across for P11

4.23 Functional connectivity networks corresponding to pre- inter- post- ictal

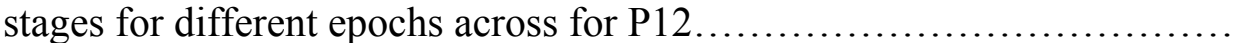

4.24 Graph Theory Measurements corresponding to pre- inter- post- ictal stages

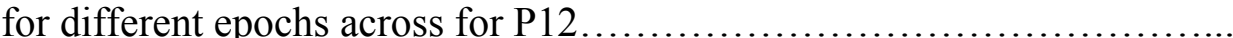

4.25 Functional connectivity networks corresponding to pre- inter- post- ictal stages for different epochs across for P13....

4.26 Graph Theory Measurements corresponding to pre- inter- post- ictal stages for different epochs across for P13

4.27 Functional connectivity networks corresponding to pre- inter- post- ictal stages for different epochs across for P14.

4.28 Graph Theory Measurements corresponding to pre- inter- post- ictal stages for different epochs across for P14....

4.29 Functional connectivity networks corresponding to pre- inter- post- ictal stages for different epochs across for P15....

4.30 Graph Theory Measurements corresponding to pre- inter- post- ictal stages for different epochs across for P15.

4.31 Mean and standard deviations of three network measures of graph theory based FCN's maps for different conditions (pre, inter and post)...........

5.1 Overview of the study protocol

5.2 Reference ICV segmentation for a sample sagittal slice of randomly selected subject from each group.

$5.35^{\text {th }}, 25^{\text {th }}, 50^{\text {th }}, 75^{\text {th }}$, and $95^{\text {th }}$ percentiles of the empirical distribution of intra-class correlation coefficient and Maximum Percentage Error

5.4 Plot of ICV measurements through automated tools (FS, FSL and SPM) 
against ICV measurements performed

5.5 Intra-software variations in automated ICV measurement of each software (FS, FSL and SPM) are shown as box plots in phase I of the study and phase II of the study across each subject group (PE, PC, AD

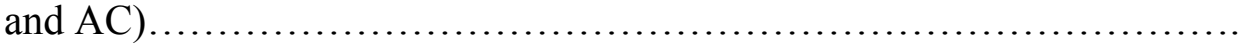

6.1 Constructed functional connectivity map (the threshold of $45^{\circ}$ is applied as the connection strength) for (a) subject diagnosed with left frontal region epilepsy and (b) subject diagnosed with generalized epilepsy........

6.2 Mean Related Percentage Absolute Difference (MRPAD) between reference ICV and estimated ICV using FSL with varying Fractional Intensity Threshold (-f parameter) in the range of 0.1 to 1 with steps of 0.1 across each subject group (PE, PC, AD and $\mathrm{AC}$ ) (Left) Corresponding plots of errors (SB: Systematic Bias; RE: Randomized Error mean) (Right) 


\section{SYMBOLS AND ABBREVIATIONS}

\begin{tabular}{ll} 
ECG & Electrocardiography \\
EEG & Electroencephalography \\
EMG & Electromyography \\
FCN & Functional Connectivity Network \\
FCD & Focal Cortical Dysplasia \\
GLM & General Linear Model \\
GMM & Gaussian Mixture Model \\
ICA & Independent Component Analysis \\
ICC & Intra-class Correlation Coefficient \\
ICV & Intracranial Volume \\
MAP & Maximum A Posteriori \\
MLE & Maximum Likelihood Estimation \\
MPE & Maximum Percentage Error \\
MRI & Magnetic Resonance Imaging \\
MRPAD & Mean Relative Percentage of Absolute Difference \\
PC & Pediatric Control \\
PE & Pediatric Epilepsy \\
RF & Radio Frequency \\
SFS & Sequential Feature Selection \\
\hline
\end{tabular}




\section{CHAPTER 1}

Introduction

\subsection{General Statement of Problem Area}

The Center for Disease Control and Prevention (CDC) estimates that more than 2.3 million adults and half a million children in the United States are affected by epilepsy (Control and Prevention 2012), which is characterized by the occurrences of recurrent seizures with unknown causes. Around 50 million people worldwide have epilepsy, according to the World Health Organization. This number is projected to increase every year, with 0.15 million newly diagnosed epilepsy cases in the US alone. Although the impact of seizures varies from person to person, physical and mental functions of the affected person could be severely altered. A systematic approach in diagnosing epilepsy could inform clinicians on ways to improve the treatment process and provide patientspecific care. Scalp Electroencephalography (EEG) has been widely perceived as an effective tool for non-invasive study of the brain in individuals with epilepsy. High temporal resolution makes of EEG recordings an indispensable tool in offering initial diagnosis of epilepsy followed by detailed analysis of the characteristic temporal events like interictal spikes, which are closely associated with epileptic foci (Cabrerizo, Ayala et al. 2012). However, visual inspection and interpretation of continuous temporal EEG recordings is tedious, time consuming and prone to human error. Furthermore, epilepsy diagnosis based on visual inspection of EEG has been shown to be very subjective to the expert opinion (Kirmani 2013). This has led to a cohort of automated techniques for diagnosing epilepsy. Epilepsy, being a complex disease with multifactorial causes, makes the diagnostic process much more complicated than simply relying on isolated inspection 
of scalp EEG electrode recordings. In fact, the human brain includes a complex web of neuronal interconnectivity and discrete anatomical regions that function together to generate brain activity. This underlying brain infrastructure suggests that solutions to epileptic diagnosis need to consider the whole brain network together with the resulting functional inter-connections, referred to as connectivity networks.

Connectivity networks seek to define a pattern of cross-correlation between discrete functionally characterized brain regions associating statistical importance to anatomical connectivity and subsequently determining inter-regional neurophysiological inferences. The hypothesized belief is that epilepsy alters the pattern of connectivity network in both functional and structural contexts. For instance, such effects could be investigated to distinguish epileptic subjects from healthy controls by extracting the pattern of functional and structural connections among different brain regions. Functional connectivity can be assessed by measuring the amount of shared information among scalp EEG electrodes or through functional Magnetic Resonance Imaging (fMRI); whereas connectivity networks constructed by extracting morphometric features from MRI imaging are most commonly employed to study the structural connectivity. These functional and structural connectivity networks can together be explored to study and enhance the assessment of the differential brain patterns that could delineate patients with a specific neurological disorder from normal controls.

Although connectivity has shown great promise towards classification and understanding of characteristic features in patients with neurological disorders, the dependence of regional structural features, such as cortical volume, cortical thickness and cortical 
surface areas on variables, such as head size (especially in a pediatric population) has limited its full potential attainments. An important step to be considered before any structural or functional connectivity patterns can be analyzed is the need to correct for head size. Magnetic Resonance Imaging (MRI) based estimation of intracranial volume (ICV) has been accepted as an excellent measure for the purpose of inter-subject head size correction/normalization. Lack of a validated technique and guideline to accurately estimating ICV in any neurological disorder is an issue that is yet to be resolved and is investigated thoroughly in this dissertation.

\subsection{Research Purpose}

The research objectives of this dissertation include:

- Developing a data-driven approach for constructing brain functional connectivity networks (FCNs) using time domain analysis of scalp EEG recordings.

- Providing graph theoretical analysis of FCNs with application to epilepsy.

- Investigating the functional network topology and characteristics in the evolution of interictal events.

- Determining a methodology for effective manual and automatic ICV estimation.

\subsection{Significance of the Study}

Data-driven methods on the basis of functional connectivity networks could provide an unbiased approach with the potential for effective applicability in epilepsy diagnosis. Such data-driven methods do overcome the reliance on the training phase for classification purposes as well as provide solutions that are independent of the variability 
in diagnostic opinions provided by the different raters. Thus, such methods on the basis

of the structural and functional regions could significantly improve our understanding of epilepsy pathways, especially when combined with the existing knowledge of source localization and the 3D context of delineated tumors or lesions. Furthermore, an integral part of the project to determine a validated protocol for accurate estimation of ICV in the pediatric epilepsy population will serve to reduce the bias on the constructed structural network due to head size and age differences.

\subsection{Research Questions and Hypotheses}

The following are the main hypotheses that are assumed within the research context of this dissertation, along with the research questions that are posed.

Hypothesis (a) Functional connectivity networks of pediatric epilepsy subjects are found to be statistically different from normal controls using patient-specific scalp EEG recordings.

Question (a) Is pediatric epilepsy associated with any changes in the functional brain connectivity network as derived from scalp EEG recordings of the resting state? If yes, what topological features are statistically different between pediatric epilepsy subjects and normal controls?

Hypothesis (b) Functional connectivity network measures (clustering coefficient, global efficiency and small world index) of pre-ictal duration are not found to be statistically different from post-ictal duration but different from interictal duration. 
Question (b) Are there any similarities between the topology and graph theoretical measures of functional connectivity networks constructed at the onset of different ictal events for the same subject? How are the functional connectivity maps at the onset and during the ictal events related to the foci of epilepsy? How do FCN measures such as small-world index vary before, during and after the ictal event?

Hypothesis (c) The choice of the right protocol for ICV estimation of different populations has statistically significant impact on the accuracy.

Question (c) Are populations' health condition, sex, age and the choice of a particular protocol for ICV estimation related to the accuracy in estimating ICV? What is the right protocol to assure accurate estimation of ICV as a function of the population under study?

\subsection{Structure of the Research}

The two main research themes of this dissertation can be summarized as follows:

(1) Design of a methodology for constructing functional connectivity networks used for the study of hypothesized changes due to epilepsy and the evolution of ictal events.

(2) Evaluation of standard protocols for ICV estimation across different populations for effective structural network analysis.

Consequently, this dissertation is structured in the following way: 
Chapter 2 provides a review of relevant literature related to ICV estimation and brain functional connectivity networks, and explains the basic mathematical framework used in context to the objectives of this dissertation. It outlines also the main elements in making use of the scalp EEG and MRI recording modalities. General Linear Model (GLM) is explained as it is used in the first version of the proposed functional connectivity network (FCN) for pediatric epilepsy diagnosis when a priori evaluation of the diagnosis is made available. Gaussian Mixture Model (GMM) and Maximum Likelihood Estimation (MLE) are overviewed as they are the foundation of the extended version of the proposed FCN-based technique for pediatric epilepsy diagnosis when no prior knowledge of the diagnosis is available. This chapter briefly discusses the concept of graph theory and the network measures which have been utilized to assess the FCNs.

Chapter 3 details application and implementation of the aforementioned methods, namely the GLM, the GMM, and the MLE for pediatric epilepsy, contrasting the diagnostic outcome when a priori evaluation of the diagnosis is available or not. The topologies of both versions are analyzed from the graph theory perspective and their accuracy in delineating the patient population from the control population is discussed in this chapter.

Chapter 4 addresses the meaningfulness of FCNs and discusses the hypothetical similarities between the FCNs as they relate to the onset of ictal events across the same subject. It also investigates the time-varying analysis of the FCNs during the ictal events. This is extremely important in the case of longitudinal studies trying to assess the merits of medication and other therapeutic interventions and their effect over time on the neurological disorder. 
Chapter 5 provides a comprehensive reliability assessment of currently existing protocols, viewed as standard protocols for ICV estimation. They are evaluated across four different populations, namely Pediatric Control (PC), Pediatric with Epilepsy (PE), Adult Control (AC) and Adults with Alzheimer's disease (AD). It then follows with guidelines and recommendation in seeking processes that yield accurate ICV estimations in both manual and automated modes.

Chapter 6 finally concludes this dissertation with a discussion on the findings and their implication to potential future extensions. 


\section{CHAPTER 2}

\section{Literature Review and Design Fundamentals}

\subsection{Literature Survey}

The literature review as it relates to this dissertation main research themes is divided into the following main sections:

- Brain functional connectivity networks with applications to epilepsy

- ICV estimation across different populations with applications to epilepsy and Alzheimer's disease.

\subsubsection{Brain Functional Connectivity Networks}

Epilepsy is a neurological disorder characterized by recurrent seizures with unspecified causes. The Center for Disease Control and Prevention (CDC) estimates that more than 2.3 million adults and half a million children in the United States are affected by Epilepsy (Control and Prevention 2012). This number is projected to dramatically increase every year with 0.15 million newly diagnosed epilepsy cases (England, Liverman et al. 2012). Although the impact of seizures varies from person to person, physical and mental functions of the affected person could be severely altered. Scalp Electroencephalography (EEG) recording at resting state condition has been widely perceived as an effective preliminary tool for non-invasive study of the brain in individuals with epilepsy. Analysis of scalp EEG during resting state condition without performing a cognitive task and with the absence of external stimuli, has gained significant prominence for assessing brain function and related disorders. Applications include surveying the response of the brain 
under the influence of drug therapies (Alonso, Mañanas et al. 2006), source localization of epileptic seizures which exploits techniques in the time/ frequency domains for analysis of individual EEG electrode recordings (Adjouadi, Sanchez et al. 2004; Adjouadi, Cabrerizo et al. 2005). Assessment of brain functional connectivity networks in patients suffering from various neurological disorders through modalities such as EEG, Magnetoencephalography (MEG) and functional Magnetic Resonance Imaging (fMRI) have elicited new findings that allow for the delineation of epileptic patients from a control population (Sargolzaei, Cabrerizo et al. ; Lowe, Mock et al. 1998; Cabrerizo, Ayala et al. 2012; Eddin, Wang et al. 2013; Sargolzaei, Cabrerizo et al. 2013; Sargolzaei, Eddin et al. 2013; Ahammad, Fathima et al. 2014; Sargolzaei, Goryawala et al. 2014; Xie and Krishnan 2014). The high temporal resolution of EEG renders it as an indispensable tool in the primary diagnosis of epilepsy and in the visualization of temporal events like interictal spikes which are closely associated with epileptic foci (Cabrerizo, Ayala et al. 2012; Kim, Faloutsos et al. 2013). Additionally, EEG has been utilized to distinguish focal and generalized neurophysiologic correlates of epilepsy (Barkley and Baumgartner 2003). When not automated, diagnosis of epilepsy by means of scalp EEG will often involve visual inspection of long term recordings, and ensuing investigation by the EEG expert is performed in search of abnormal activities and specific characterizing events.

Consequently, visual inspection of continuous temporal EEG recordings is often tedious, time consuming and prone to human error. Furthermore, epilepsy diagnosis based on visual inspection of EEG has been shown to be very subjective to the expert opinion (Kirmani 2013). This has led to the general cohort of adopting various computer aided 
techniques with the help of machine learning for medical applications (Sargolzaei, Faez et al. 2008; Sargolzaei, Faez et al. 2009; Sargolzaei, Faez et al. 2011). Artificial Neural Network (ANN) based classifiers have received the most attention towards epilepsy diagnosis using scalp EEG recordings (Subasi and Erçelebi 2005; Guo, Rivero et al. 2010; Cabrerizo, Ayala et al. 2011; Cabrerizo, Ayala et al. 2011; Subasi, Alkan et al. 2005; Subasi and Erçelebi 2005) have reported high classification rates. The general routine of ANN based techniques, which rely on a training phase and a priori knowledge, is to process each isolated EEG signal with the aim of extracting a set of discriminating features as input to train an ANN towards the design of an optimal classifier. Considering the fact that Epilepsy is a complex disease with multifactorial causes, makes the diagnostic process much more complicated than simply relying on solely model driven knowledge. Furthermore, human brain involves a complex web of neuronal connectivity and discrete anatomical regions that function together to generate brain activity (Lowe, Mock et al. 1998; Eddin, Wang et al. 2014).

This underlying functional brain infrastructure suggests that solutions to epilepsy diagnosis need to consider the whole brain network with the pattern of functional connections, which is referred to as functional connectivity networks (FCNs). Thus, FCNs seek to define the patterns of cross-correlation between discrete functionally characterized brain regions to give statistical importance to anatomical connectivity (upon the existence of physical connection) and subsequently determining inter-regional neurophysiological inferences. FCNs could be grouped into two broad categories: directed and undirected. Undirected FCNs finds the dynamic associations among 
functional brain regions without considering the hypothetical causalities among them. Whereas Directed FCNs, sometimes referred to as effective connectivity (Lang, Tomé et al. 2012), assesses the influence of one cerebral region upon another and therefore gives direction to the calculated associations. Current trends in adopting FCNs for understanding the complex brain are placed toward developing data driven methodologies for constructing FCNs which benefits from a robust parcellation of functional data of the brain and an objective formulation of the hypothesized association among functional parcels (Lang, Tomé et al. 2012; Sargolzaei, Eddin et al. 2013). The crucial role of time delay in the dynamics of large scale networks (Sargolzaei, Yen et al. 2013) such as brain networks is well motivated, due to the large scale property of brain connectivity networks including discrete sub-networks (Jirsa 2004), but yet not fully understood and incorporated in constructing the brain networks and decision making processes (Ghosh, Rho et al. 2008). Time delay is coupled with the fact that on large scale networks such as brain networks, the recorded signal at each electrode is in fact showing the summation of the variance of the brain area closer to the electrode and a lagged version of variances from other brain areas.

In this dissertation, new algorithms based on associations among channels of scalp EEG using a moving window is examined for its ability to identify multichannel EEG segments with epilepsy driven characteristics with or without a priori knowledge assumed for the diagnosis. Undirected FCNs estimate the association between the channels of EEG using a geometrical distance between a pair of channels. Undirected FCNs can be represented in the form of undirected graphs. Each electrode is considered 
as a node and the functional associations among them are the edges of the corresponding graph. Different applications of graph theory and small world networks (Smit, Stam et al. 2008), with causality analysis combined with network analysis (Friston 1994) and timefrequency coupling detection among isolated scalp EEG recordings (Adjouadi, Cabrerizo et al. 2005; Cabrerizo, Adjouadi et al. 2005) are a few of the widely used model-based solutions for studying FCNs using scalp EEG recordings. This dissertation also assesses the merit of graph theory based features, extracted from the graphs corresponding to the FCNs, in identifying EEG segments recorded from patients with epilepsy from segments recorded from the pediatric control group. The main contribution of the study is in introducing a novel approach based on machine learning techniques to facilitate the screening process of potential epileptic patients.

\subsubsection{Intracranial Volume Estimation}

Intracranial volume (ICV), sometimes called total intracranial volume (TIV), refers to the estimated volume of the cranial cavity as outlined by the supratentorial dura matter or cerebral contour when dura is not clearly detectable (Eritaia, Wood et al. 2000). ICV is often used in studies involved with analysis of the cerebral structure under different imaging modalities, such as Magnetic Resonance (MR) (Ehrlich, Morrow et al. 2010; Kochan, Breakspear et al. 2011), MR and Diffusion Tensor Imaging (DTI) (Groves, Smith et al. 2012), MR and Single-photon Emission Computed Tomography (SPECT) (Garibotto, Borroni et al. 2011), Ultrasound (Graca, Cardoso et al. 2013) and Computed Tomography (CT) (Ritvanen, de Oliveira et al. 2013; Sonmez, Temel et al. 2013). ICV

consistency during aging (Ikram, Fornage et al. 2012) makes it a reliable tool for 
correction of head size variation across subjects in studies that rely on morphological features of the brain. ICV, along with age and gender are reported as covariates to adjust for regression analyses in investigating progressive neurodegenerative brain disorders, such as Alzheimer's disease (Fennema-Notestine, Hagler et al. 2009; Piguet, Petersén et al. 2011; Dukart, Mueller et al. 2013; Lampert, Choudhury et al. 2013; Westman, Aguilar et al. 2013), aging and cognitive impairment (Trivedi, Stoub et al. 2011). ICV has also been utilized as an independent voxel based morphometric feature to evaluate age-related changes in the structure of premorbid brain (Szentkuti, Guderian et al. 2004; Cardenas, Chao et al. 2005; Peper, Schnack et al. 2009; Roussotte, Sulik et al. 2012; Taki, Thyreau et al. 2013), determine characterizing atrophy patterns in subjects with mild cognitive impairment (MCI) and Alzheimer's disease (AD) (Pa, Boxer et al. 2009; Thambisetty, An et al. 2012), delineate structural abnormalities in the white matter (WM) in schizophrenia (Cullen, Wallace et al. 2012), epilepsy (Gong, Alexander et al. 2012), and gauge cognitive efficacy (Chee, Zheng et al. 2011).

For the existing protocols for calculating ICV, despite their methodological differences, they can be classified mainly into two broad categories, manual and automated. Manual estimation of ICV involves segmentation of the cranial cavity by hand in every single slice of brain volume. The process of manual segmentation of ICV is a tedious and lengthy process. In order to alleviate this process, different sampling protocols as opposed to considering every slice were suggested and evaluated previously (Eritaia, Wood et al. 2000). Calculating ICV following the subsampling protocol (Eritaia, Wood et al. 2000) reported that no significant loss of measurement reliability (0.999) was observed by segmenting ICV every 10 sagittal slices with $0.938 \mathrm{~mm}$ thickness instead of measuring 
ICV in every single slice. Although subsampling strategies result in significant time saving, this finding was limited to the normal control adult population. Consequently, the first aim of the current study focused on evaluating subsampling protocols for manual estimation of ICV in the following four different subject categories: control pediatrics, control adults, pediatrics with epilepsy and adults with Alzheimer's disease. The choice of these groups were based on the importance of the ICV measure as a critical normalization factor whether the study is based on AD (Jack, Knopman et al. 2012; Jack Jr, Knopman et al. 2013; Westman, Aguilar et al. 2013; Zhou, Goryawala et al. 2014) or on epilepsy (Whitwell, Crum et al. 2001; Wang, Zhang et al. 2012; Winston, Cardoso et al. 2013; Germeyan, Kalikhman et al. 2014).

Automated approaches for estimating ICV are highly desirable in order to minimize the level of manual intervention required from the human rater in the estimation procedure. Freesurfer (Dale, Fischl et al. 1999), FSL (Jenkinson, Beckmann et al. 2012) and SPM (Ashburner and Friston 2005) are three widely used software packages in neuroimaging studies, which come with their own routines for estimating ICV. Accuracy of the software packages in estimating ICV has recently been investigated (Nordenskjöld, Malmberg et al. 2013). The main challenge in this reliability assessment is in determining if the estimated ICV through each package is consistent over the variability exhibited with respect to age population (adult or pediatric), strength of the magnetic field in case of MR based imaging, slice thickness, condition of the population targeted (control or patient) and the type of the neurological disorder (Pengas, Pereira et al. 2009; Nordenskjöld, Malmberg et al. 2013). Challenges with regards to ICV estimation using different field strengths (Keihaninejad, Heckemann et al. 2010), and in estimating ICV in 
adult subjects with dementia as the neurological disorder (Pengas, Pereira et al. 2009; Ridgway, Barnes et al. 2011) have been well addressed in these studies. However these type of evaluations which focused on the use of two software platforms (Freesurfer and SPM) and involved only adult populations, were shown to upwardly bias the ICV for adult subjects (Nordenskjöld, Malmberg et al. 2013), and Freesurfer and SPM 5 in the case of subject with dementia (Ridgway, Barnes et al. 2011) have shown an overestimation of the ICV by Freesurfer. Therefore, it is important to compare the performance of the software on ICV estimation inclusive of both pediatric and adult groups and assuming different neurological disorders, in this case AD and epilepsy.

\subsection{Scalp Electroencephalography}

Scalp Electroencephalography (EEG), recording of electrical activity of brain along the scalp, has been recognized widely as a tool for the non-invasive study of the brain in individuals with epilepsy (Sargolzaei, Cabrerizo et al. 2013) in clinical routines. Scalp EEG recording is obtained by placing electrodes on the scalp, usually preceded by some forms of skin preparation depending on the technology used. International 10-20 system (Towle, Bolaños et al. 1993) is commonly used as the electrode placement guideline for clinical and research EEG recordings. Figure 2.1 shows the locations of 19 electrodes recordings used in our study following the international 10-20 electrode placement system. The letters match the anatomical lobe which the corresponding electrode belongs to. The figure has been generated using BrainNet Viewer tool (Xia, Wang et al. 2013). 


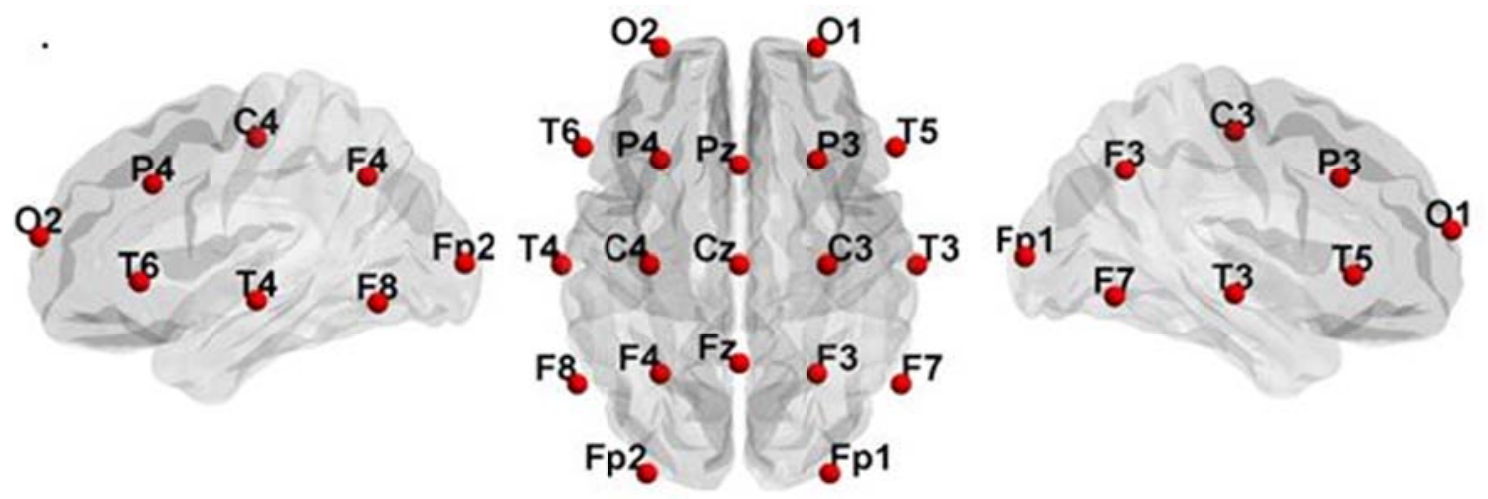

Figure 2.1: International 10-20 electrode placement system. F: Frontal; C: Central; P: Parietal; T: Temporal; O: Occipital. Odd numbers correspond to left hemisphere and even numbers correspond to right hemisphere.

Referential montage and Average montage are two widely used modes of representation and analysis of EEG signals. Referential refers to when voltage differences between the electrode and a reference electrode e.g. $\mathrm{Cz}$ is measured whereas Average montage uses the average of all electrodes as the reference measurement. EEG has gained significant prominence for studying the response of the brain under the influence of stimulations; for neuro-psycho-pharmacology study of drug therapy s(Alonso, Mañanas et al. 2006; Schoffelen and Gross 2009; Oostenveld, Fries et al. 2011); for source localization of epileptic seizures using interictal spike (Adeli, Zhou et al. 2003; Adjouadi, Sanchez et al. 2004; Adjouadi, Cabrerizo et al. 2005) and other applications which exploit techniques using time/frequency analysis of individual EEG electrode recordings. Scalp EEG is the modality used for FCN construction in chapters 3 and 4.

\subsection{Magnetic Resonance Imaging}

Magnetic Resonance Imaging (MRI) is a non-invasive three dimensional imaging technique based on the magnetization characteristics of tissues different. It is widely 
utilized in anatomical studies of brain (Ogawa, Lee et al. 1990) including structural connectivity networks. The fundamentals of MRI is too extensive beyond the scope of this dissertation, however a few related terminology are defined (Sargolzaei 2012) as they are referred to in chapter 5 .

MRI Scan: $\quad$ A non-invasive three dimensional imaging technique.

MRI Sequence: A special collection of Radio Frequency (RF) pulses set to provide contrast on a particular tissue signal.

Localizer Sequence: A sequence involving low resolution template images to locate the slices in right position in three planes (Axial, Sagittal and Coronal).

Axial plane: $\quad$ Imaging plane bisecting the body into top and bottom sections.

Sagittal plane: $\quad$ Imaging plane bisecting the body into right and left sections.

Coronal plane: $\quad$ Imaging plane bisecting the body into front and back sections.

T1: A biological parameter which represents the time required for a tissue type to release the energy and realign with the external field.

MPRAGE: $\quad$ Magnetization Prepared Gradient Echo, a fast three dimensional acquisition with $\mathrm{T} 1$ weighted dominance.

Slice thickness: The thickness of an imaging slice.

Voxel: $\quad$ A single unit in volumetric imaging.

\subsection{General Linear Model}

General Linear Model (GLM) is the term used for multiple regression analysis (Weisberg 2005) aiming to explain the variation of a dependent (response) variable in terms of linear 
combination of independent (explanatory) variables. GLM has been extensively the analysis core of functional MRI experiments (Sargolzaei 2012; Salah Eddin 2013). GLM system of equation could be expressed as

$$
y=X b+e
$$

where $X$ is the explanatory variable, $y$ is the dependent variable, $e$ is the residual error and $b$ is known as beta parameters which is to be estimated. Different techniques such as maximum likelihood or Bayesian approaches are available to estimate the unknown beta parameters (McCullagh, Nelder et al. 1989). Least square estimate of beta parameters of GLM could be expressed as

$$
b=\left(X^{t} X\right)^{-1} X^{t} y
$$

where $X^{t}$ is the transpose of $X$ and $\left(X^{t} X\right)^{-1}$ is the inverse of $X^{t} X$. GLM is utilized in chapter 3 in the first version of FCN based pediatric epilepsy diagnosis scheme.

\subsection{Gaussian Mixture Model}

Gaussian Mixture Model (GMM) is used to parametrically model the probability distribution of a random variable as a weighted sum of components with Gaussian probability distribution. GMM is commonly used in natural audio processing and speaker recognition systems(Reynolds 2009; Ziaei, Sangwan et al. 2012). A GMM of $M$ components is mathematically expressed as

$$
p(x \mid \lambda)=\sum_{i=1}^{M} \omega_{i} g\left(x \mid \mu_{i}, \Sigma_{i}\right)
$$


where $x$ is the $D$-dimensional measurement vector and $g\left(x \mid \mu_{i}, \Sigma_{i}\right)$ is the Gaussian density distribution of $i$ 'th $D$-variate Gaussian component which has the expected value of $\mu_{i}$ and covariance of $\Sigma_{i} . \omega_{i}$ is the mixture weight corresponding to $i$ 'th component and with other weights satisfies the following constraint:

$$
\sum_{i=1}^{M} \omega_{i}=1
$$

Each component $g\left(x \mid \mu_{i}, \Sigma_{i}\right)$ has a form of Gaussian distribution as shown in equation (2.5):

$$
g\left(x \mid \mu_{i}, \Sigma_{i}\right)=\frac{1}{(2 \pi)^{\frac{D}{2}}\left|\Sigma_{i}\right|^{\frac{1}{2}}} \exp \left(-\frac{1}{2}\left(x-\mu_{i}\right)^{t} \Sigma_{i}^{-1}\left(x-\mu_{i}\right)\right)
$$

$\lambda$ is representing the complete GMM parameterized by the mean vectors, covariance matrices and the mixture weights of $D$-variate Gaussian components in the form of

$$
\lambda=\left\{\omega_{i}, \mu_{i}, \Sigma_{i}\right\} \quad i=1,2, \ldots, M
$$

GMM parameters could be estimated through Maximum Likelihood Estimation (MLE) and Maximum A Posteriori (MAP) estimation techniques (Reynolds 2009). GMM is used in the second version of pediatric epilepsy diagnosis scheme proposed in Chapter 3 in the case that no prior knowledge on the diagnosis is available.

\subsection{Graph Theory and Network Measures}

Graph theory is a well-established and rich source of benchmarks for studying functional connection as well as anatomical connections in the brain (Van Wijk, Stam et al. 2010). Directed FCNs and undirected FCNs in terms of connectivity strength among pair of 
scalp EEG channels recordings could be mathematically represented and studied in the forms of directed and undirected network graphs (Eddin, Wang et al. 2014). Pairwise relations between objects could be mathematically modeled and studied in terms of a graph. Nodes of the graph refer to the electrodes used to record EEG signals and their location in graph is determined from the coordinates in the 10-20 EEG electrode placement system. An edge is defined as the pairwise relation connecting a pair of nodes (electrodes in the context of the study). Pairwise angles which make up the adjacency matrix are termed as weights of graph edges for the study. The graph as defined here is an undirected form of FCN. A graph may not include edges among pair of nodes when there is no communication between them.

Graph theoretical analyses of multi-channel EEG have been evaluated for different schemes of complex brain networks affected by epilepsy (Ponten, Bartolomei et al. 2007; Smit, Stam et al. 2008; Ponten, Douw et al. 2009; Van Dellen, Douw et al. 2009; Seth 2010), emphasizing that small world network model of the brain function could be altered by epilepsy. Graph theory has been utilized to assess the dynamics of the brain networks. Measuring the network characteristics has been mostly directed with a set of MATLAB tools for network analysis (Bounova and de Weck 2012).

- Link density of a Graph: computes the density of the links in the graph, calculated as number of edges divided by the maximum possible number of edges.

- Degree of node: number of links connecting one node to the others in a graph.

- Closeness centrality: closeness centrality of a vertex is defined as the inverse of sum of distances to all other nodes. 
- Betweenness centrality: It measures the local characteristics of a graph (Salah Eddin et al. 2013). Betweenness centrality of a vertex is the ratio of the number of shortest path lengths for which the vertex is part of to the total number of shortest paths.

- Characteristic path length: Average of all shortest path lengths between all pairs of vertices in a graph.

- Clustering coefficient: an average of all node's clustering coefficients in the graph. Clustering coefficient of a node is the ratio of the number of edges corresponding to that node to the total number of edges within the node's neighborhood.

- Rich club metric: It is a representation of the graph's tendency of high degree nodes to link to each other.

- S-metric: The sum of products of degrees across all vertices in the graph.

- Algebraic connectivity: Algebraic connectivity of a graph is the second smallest eigenvalue of the Laplacian of the adjacency matrix corresponding to the graph.

- Graph energy: The sum of absolute values of real components of the eigenvalues of the corresponding adjacency matrix is interpreted as the graph energy.

- Small-world index: Large scale networks could yield two extreme topological perspectives. One could have a high clustering coefficient and relatively long path length and the other could have a low clustering coefficient and shorter path lengths. Small-world network topology keeps the middle ranges with high clustering coefficient and short path length. A network is evaluated for its tendency to be small word by measuring two sets of ratios. Clustering coefficient of the network needs to be greater than clustering coefficient of random networks but its characteristic path length is required to be similar to the one of random networks. 


\section{CHAPTER 3}

\section{Brain Functional Connectivity Network}

\subsection{Introduction}

Patients with epilepsy are severely affected due to the alterations in their functional and mental abilities which epilepsy causes. This chapter aims to introduce novel decision support systems for the diagnosis of pediatric epilepsy based on scalp EEG data in clinical environment. It first focuses on the construction of brain functional connectivity networks on the basis of these EEG recordings. Constructed FCN's are utilized to study the hypothetical alterations due to epilepsy in pediatric population. Two new algorithms were proposed and evaluated in terms of their classification accuracy.

\subsection{Algorithm I: GLM-based Approach}

\subsubsection{Data Acquisition}

16 children (7 pediatric control (PC) and 9 pediatric epilepsy (PE) patients) were recruited for this study. Scalp EEG obtained with varying sampling frequencies of 200 $\mathrm{Hz}, 500 \mathrm{~Hz}$ and $512 \mathrm{~Hz}$ from control subjects (4 males and 3 females) and patients with

epilepsy (5 males and 4 females) were recorded using the 10-20 electrode placement system with a referential montage. The raw digital EEG data used one reference electrode located in the midline of the scalp based on the $10 / 20$ system. The electrode recordings were then referenced to the average of all referential recordings. The resting state data was collected from routine EEG recordings from epileptic patients and controls, without the imposition of long recording sessions. The EEG signals were performed for screening purposes and none of the patients with epilepsy were taking medication at the time of the 
recordings. For an unbiased comparison of files, the segments from the patients with epilepsy were extracted from sections that do not include the seizure event, which may or may not contain abnormal discharges. Recordings were performed at Miami Children's Hospital, Miami, Florida, USA, using XLTEK Neuroworks Ver. 3.0.5 equipment. The file segments from patients with epilepsy were only interictal (i.e. without seizure activity). The lengths of EEG epochs free of artifacts varied from 9 seconds to 90 seconds. The choice of 9 seconds as the minimum segment size for this study followed the recommendation of (Wu and Gotman 1998) in segmenting the EEG signals in the studies focused on epileptic seizures. Table 3.1 summarizes the demographic data of study subjects. The study was approved by the Institutional Review Board and consent forms were provided to the patients or legal representatives.

\subsubsection{Preprocessing}

Continuous recorded data were digitized using an internal 22 bits analog to digital (A/D) converter and pre-processed to attenuate the effect of noise in the signal. Eye movement, muscle artifacts, eye blinks, electromyography (EMG) and electrocardiography (ECG) were labeled for artifact rejection through blind source separation artifact removal (Jung, Makeig et al. 2000) by Independent Component Analysis (ICA). Figure 3.1 outlines the main steps involved in the design of the automated decision support system to cluster a group of subjects into two categories (controls vs. patients with epilepsy).

\subsubsection{Functional Connectivity Network Construction}

The primary aim of the "Functional Connectivity Network Construction" block depicted in Figure 3.1 was to determine the pattern of functional communication among channels to establish a brain state. Structural connectivity is based on inspecting the existence of 
direct medium among brain regions found by structural imaging. Functional connectivity as defined in this study is based on the association among EEG recordings across the brain cortical regions.

Table 3.1: Demographic data of study subjects

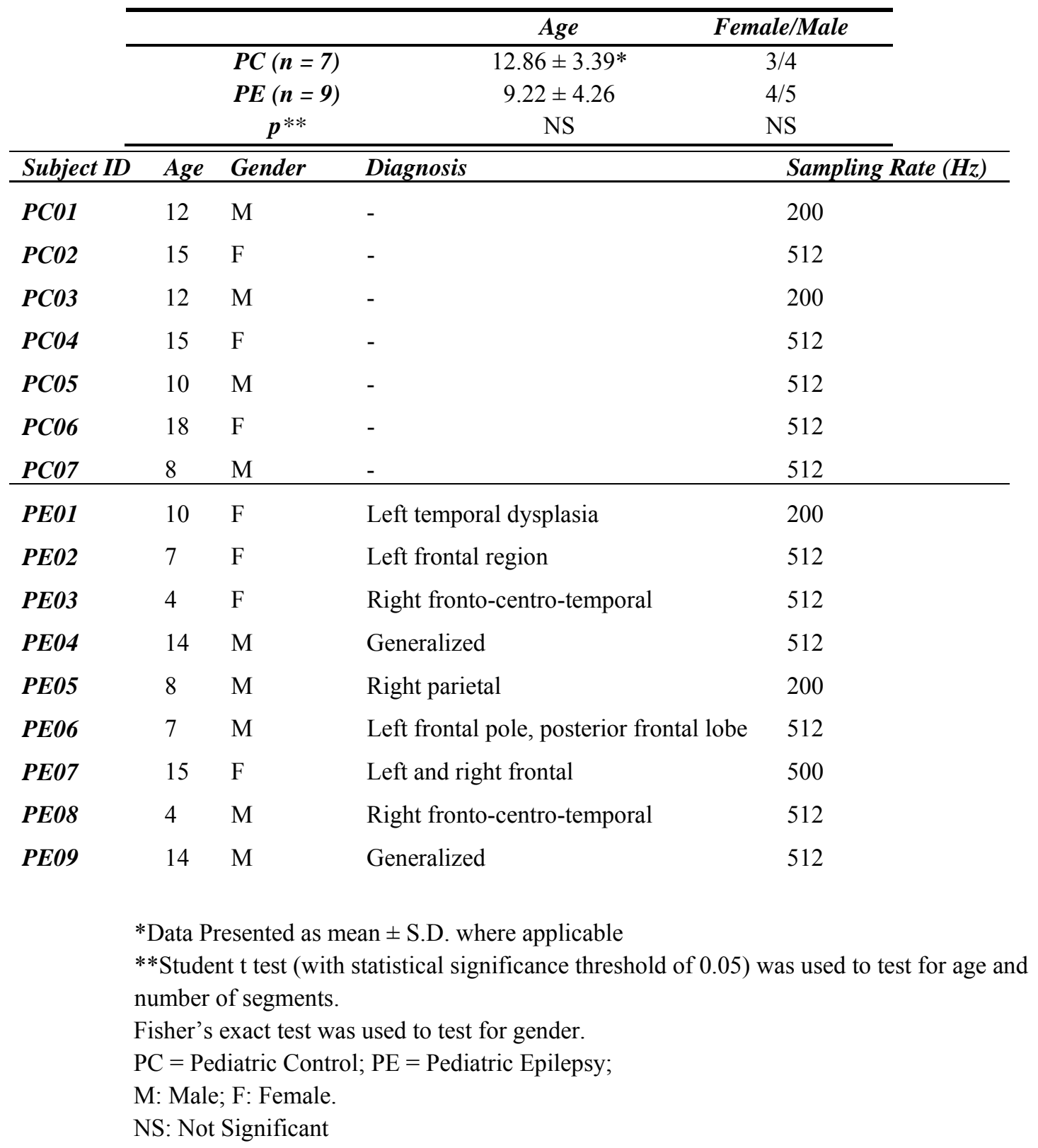




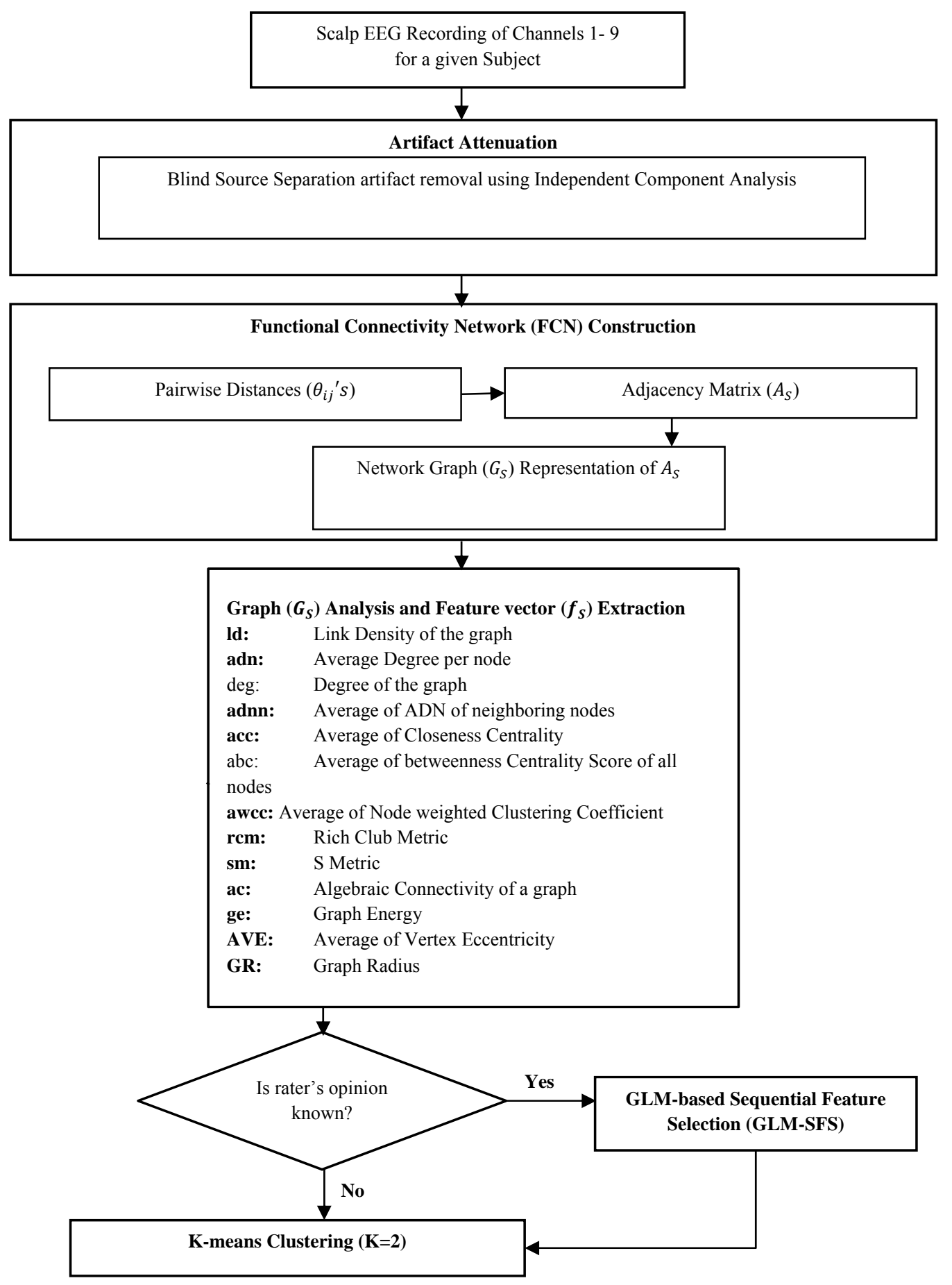

Figure 3.1: Flowchart of the suggested decision support system to differentiate pediatric with epilepsy from pediatric control. 
A variety of measures exist for defining the association among two time-series of neurophysiological signals (Pereda, Quiroga et al. 2005). Metrics such as time domain cross-correlation (Lee, Kim et al. 2006), Phase lag index (Douw, van Dellen et al. 2010), synchronization likelihood (Douw, De Groot et al. 2010) have been widely applied. Measures based on cosine similarity which is a normalized inner product are also widely accepted and applied in natural language processing and in information retrieval of text documents (Huang 2008) and multi-channel biological signals (Xu, Bakardjian et al. 2007). It is also shown that inner product based measurements e.g. cosine similarity is computationally efficient in its applications involving high dimensional sparse data (Friedman, Hastie et al. 2010; Van Durme and Lall 2010; Dhillon, Ravikumar et al. 2011). The use of more sophisticated and non-linear measures which are not based on simple cross-correlation would be definitely an option; however it is shown that almost similar efficiency was achieved with both linear and non-linear measures of mutual association among multi-channel signals when analysis of ictal and interictal signals is performed in epilepsy research (Mormann, Kreuz et al. 2005; Ansari-Asl, Senhadji et al. 2006; Quraan, McCormick et al. 2013).

To construct a functional connectivity network (FCN) each electrode recording is assigned to a vector in discrete time domain denoted as $\overrightarrow{x_{l}}, i=1,2, \ldots, m$ where $m$ is the number of electrodes. These vectors represent the EEG signals in the subject space, and their quantities at each time step are the corresponding magnitude of the signal at that time dimension. The dimension varies across subjects depending on the length of the recording. A pairwise geometrical distance $\theta_{i j} ; i, j=1,2, \ldots, m$ was defined as in 
equation (3.1) to serve as a metric in order to describe the extent by which the recordings of a pair of electrodes share mutual information in the current brain functional state.

$$
\theta_{i j}=\pi-\cos ^{-1}\left(\frac{x_{i} \cdot x_{j}}{\left\|x_{i}\right\|\left\|x_{j}\right\|}\right) i, j=1, \ldots, m
$$

This equation is essentially a distance measure calculated as a cosine similarity. Distances were normalized into $[0, \pi / 2]$ range to give a more sensible definition of vector co-variations in a geometrical context. Distance of $0 \mathrm{rad}$ corresponds to maximum

correlation among two vectors while $\pi / 2 \mathrm{rad}$ implies that the vectors are orthogonal and thus uncorrelated.

Adjacency matrices are created by concatenating all the pairwise distances $\theta_{i j}$ for each subject in a two-dimensional array, as shown in Equation (3.2).

$$
A_{S n}=\left[\theta_{i j}\right] ; i, j=1, \ldots, m
$$

The adjacency matrix for subject $n ; n=1,2, \ldots, N$ is denoted by $A_{S n}$. Adjacency matrices are thus one form for introducing the next level of automation in epilepsy classification based on graph analysis of FCN using scalp EEG measurements.

\subsubsection{Graph Analysis of FCNs}

Pairwise relations between objects could be mathematically modeled and studied in terms of a graph. Nodes of the graph refer to the electrodes used to record EEG signals and their location in graph is determined from the coordinates in the 10-20 EEG electrode placement system. An edge is defined as the pairwise relation connecting a pair of nodes 
(electrodes in the context of the study). Pairwise angles which make up the adjacency matrix are termed as weights of graph edges for the study. The graph as defined here is an undirected form of FCN. A graph may not include edges among pair of nodes when there is no communication between them.

To automatically cluster the group of subjects into epileptic or controls using the network graph $\left(G_{S n} ; n=1,2, \ldots, N\right)$ as defined from their adjacency matrices, a subset of topological features (Bounova and de Weck 2012) were extracted from the network graph. Table 3.2 lists all the features extracted from the network graphs for each subject. Features given in Table 3.2 describe density-related, distance-based and spectral features. A vector $\left(\overrightarrow{S_{S n}}\right)$ with its elements defined as the abbreviated features listed in Table 3.2 has been assigned to each subject in the following form:

$\overrightarrow{f_{S n}}=[l d$ adn deg adnn acc abc awcc rcm sm ac ge $] ; n=1, \ldots, N$

Table 3.2: List of topological features from graph corresponding to FCN

\begin{tabular}{ll}
\hline FEATURE & DESCRIPTION \\
\hline ld & Link Density of the Graph \\
adn & Average Degree per node \\
deg & Degree of the graph \\
adnn & Average of ADN of neighboring nodes for every vertex \\
acc & Average of Closeness Centrality \\
abc & Average of Betweenness Centrality Score of all nodes \\
awcc & Average of Node weighted Clustering Coefficient \\
rcm & Rich Club Metric \\
sm & S Metric \\
ac & Algebraic Connectivity of a graph \\
ge & Graph Energy \\
\hline
\end{tabular}

\subsubsection{Decision Making and GLM-based Feature Selection}

The final step of the classification system based on graph features extracted from FCN as presented in Figure 3.1 involves the use of the $K$-means clustering technique (Han and Kamber 2006). $K$-means clustering technique partitions the observation vectors $\left(f_{S j} ; j=\right.$ 
$1,2, \ldots, N)$ into $K=2$ clusters such that each observation belongs to the cluster with the closest mean. In mathematical terms, the algorithm tries to partition the $N$ feature vectors (one vector per subject) into 2 classes $C_{i} ; i=1,2$ so as to iteratively minimize the within class sum of squared differences, over all classes defined as in Equation (3.4)

$$
\operatorname{argmin}_{C} \sum_{i=1}^{2} \sum_{f_{S n} \in C_{i}}\left\|\overrightarrow{f_{S n}}-\mu_{i}\right\|^{2} \quad i=1,2 ; n=1, \ldots, N
$$

where $\overrightarrow{f_{S n}}$ refers to the feature vector assigned to subject $S n$ and $\mu_{i}$ is the centroid vector assigned to the class $C_{i}$. The difference between the feature vector $\overrightarrow{f_{S n}}$ and the centroid vector $\mu_{i}$ indicates the point-to-cluster centroid distance.

The proposed algorithm as described earlier in Figure 1 has the option to incorporate rater into the decision making process by adding a Sequential Feature Selection (SFS) block before the K-means clustering process. SFS fulfills two tasks: (1) it helps reduce the dimensionality of the observation vectors and therefore alleviating the computational burden of the algorithm; and when available (2) it integrates the rater's opinion into the decision making process.

The rule of thumb is that classification accuracy increases with increasing number of features involved, but eventually a larger number of features could decrease the accuracy due to small sample size relative to the number of extracted features (Gillies, Patel et al. 2012; Gillies, Gao et al. 2012). Non-parametric classifiers may be more prone to presence of correlation among features, however correlated features require more computation resources and add complexity to the training phase of pattern discovery. Figure 3.2 displays the pairwise correlation coefficients between each pair of features extracted from 
the graph representing FCN of each subject. Correlation coefficient is calculated and color-coded from the matrix whose rows were study subjects and columns are graph measures. Analyzing correlation coefficients suggests the high dependencies among most of the features which could lead to the curse of dimensionality issue. To overcome this situation, SFS block based on General Linear Model (GLM) (Kohavi and John 1997; Adjouadi, Cabrerizo et al. 2005; Cabrerizo, Adjouadi et al. 2005) subject to existence of clinical priori diagnosis was added to rank the features with their importance to the classification process.

The GLM-SFS process consists of first assigning a binary numeral to each subject based on the rater's opinion. The rater opinion is considered as the results of the screening process performed based on the clinical assessments. A binary numeral of ' 1 ' signifies that the subject is a patient with epilepsy whereas a ' 0 ' represents a subject from the control group. A $N \times 1$ logical vector $\vec{R}$ is thus generated for all $N$ subjects to be used in a GLM-based Sequential Feature Selection (SFS). A solution is one that fits a GLM using all features as the model repressors to the response $\vec{R}$ as in Equation (3.5). SFS then searches over all possible subsets of features and chooses the subset which gives the most accurate response in terms of mean square error.

$$
\vec{R}=F_{S_{N}} \cdot W+\varepsilon
$$

In this case, $F_{S_{N}}$ is a $14 \times N$ matrix comprising the $\mathrm{N}$ vectors $\overrightarrow{f_{S n}} ; n=1, \ldots, N$ derived earlier in equation (3.3), and $W$ are the weights to be estimated so as to minimize the error $\varepsilon$. The number of subjects involved in the study is represented by $N$. 

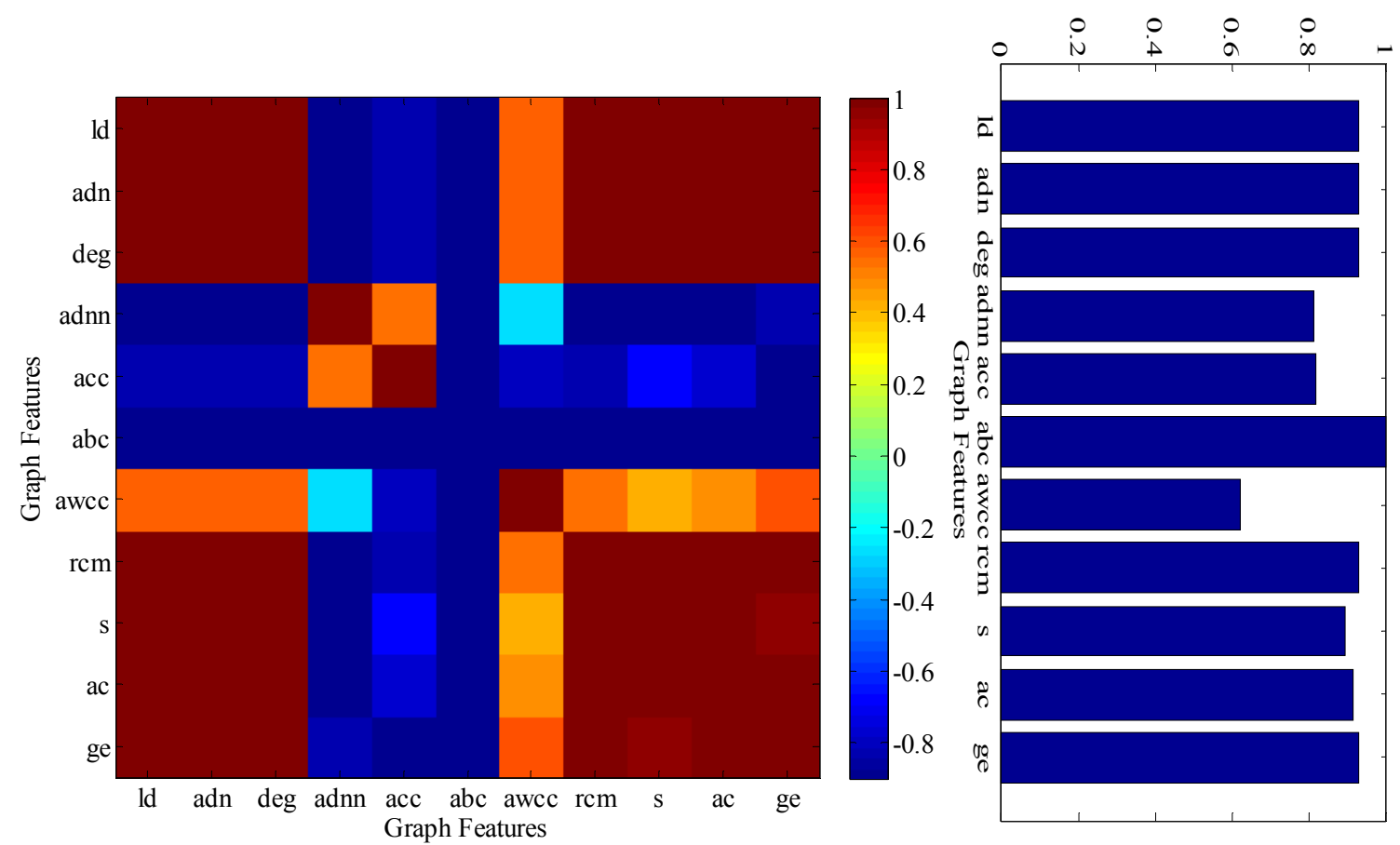

Figure 3.2: Correlation matrix (Left) Average correlation coefficient for each feature with other features (Right).

\subsubsection{Implementation Results}

Adjacency matrix $A_{S n}$ of a subject acts as the initial driver for the data-driven solution presented here for epilepsy diagnosis. Each element of the matrix is a signature of the extent by which two corresponding areas of the cortical brain, as represented by the two electrodes, are sharing common information in the current brain state. Adjacency matrix could be simply interpreted as a tool to investigate the neural interaction among different brain regions. The existence of operating functional sub-networks in the brain can be expressed by the inspection of a color-coded adjacency matrix. Furthermore a hierarchical clustering tree can be built on top of the matrix to assess the pattern of interconnections among brain regions. A set of two samples t-test were performed to inspect the existence of statistical difference among each connection between source electrode and target electrode from the set of electrodes across the pediatric control group and pediatric epilepsy group. Figure 3.3 visualizes the constructed undirected functional 
connectivity networks (FCNs) and the corresponding plot of the connectivity distances for the average map across pediatric control group (left top) and pediatric epilepsy (right top). Bottom of the figure shows the results of student $t$ test for the null hypothesis that assumes no statistically significant differences for the index pair of electrodes across the pediatric epilepsy and pediatric control groups. Rejection of the null hypothesis is highlighted with the black boxes when $p<0.00001$, Bonferroni adjusted for multiple comparison.
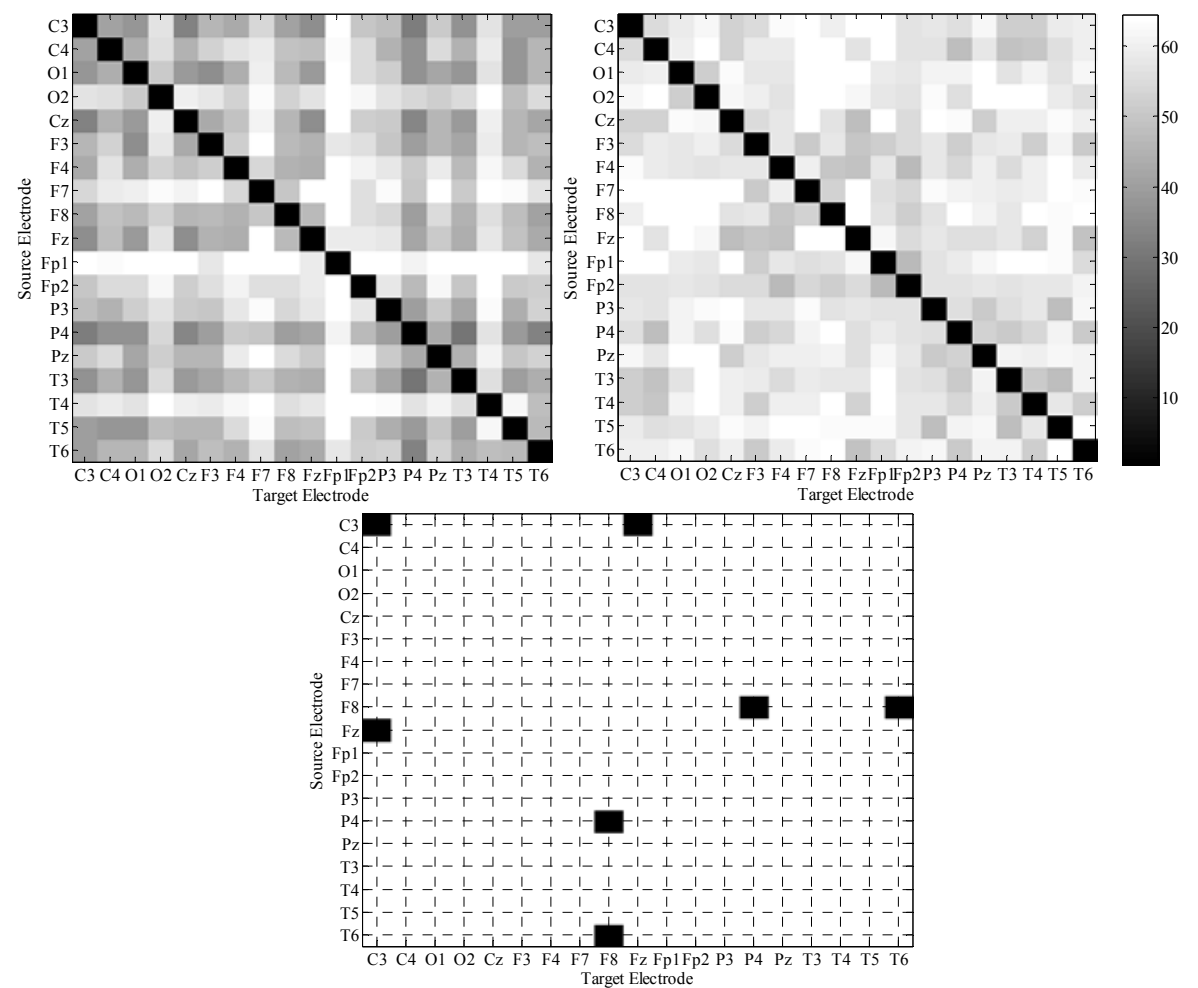

Figure 3.3: Visualization of constructed undirected functional connectivity networks.

Existence of a statistically significant difference $(p<0.00001$ Bonferroni adjusted for multiple corrections) in the connection strength (degree) of a specific source-target pair of electrodes is shown as a black box unit, insignificant difference in the connection strength is shown as a white box unit. The regional homogeneity could be observed from 
the hierarchy since the electrodes with close proximity to each other in the EEG 10-20 montage are more likely to be grouped in one sub-network. Figure 3.4 illustrates the FCN graph for an average representation of patients with epilepsy subjects and the one corresponding to the average representative of the controls group. Different threshold values were applied and the results were shown for both groups. The thickness of the edges signifies the strength of commonalities in co-variation among the pair of electrodes. The main assumption for differentiating epileptic subjects from control subjects are the differences as can be visualized in their corresponding FCNs. A twosided two-sample student t-test was conducted to compare FCN (pairwise distances) of control subjects and functional connectivity of epileptic subjects. There was a significant difference in the functional connectivity of the control subjects $\left(\mu_{C 1}=51.9^{\circ}, S D_{C 1}=\right.$ $\left.9.4^{\circ}\right)$ from the functional connectivity of the epileptic subjects $\left(\mu_{C 2}=58.86^{\circ}, S D_{C 2}=\right.$ $\left.4.93^{\circ}\right) ; t(340)=-8.57, p<0.01$. Results show that a statistical difference is seen between the FCN (pairwise distances) between the patients and control groups. Table 3.3 provides the mean and standard deviation values of each extracted feature and their corresponding two-tailed p-values of differences between the means per feature over patients with epilepsy and controls, when no thresholding was applied on the FCN's. 


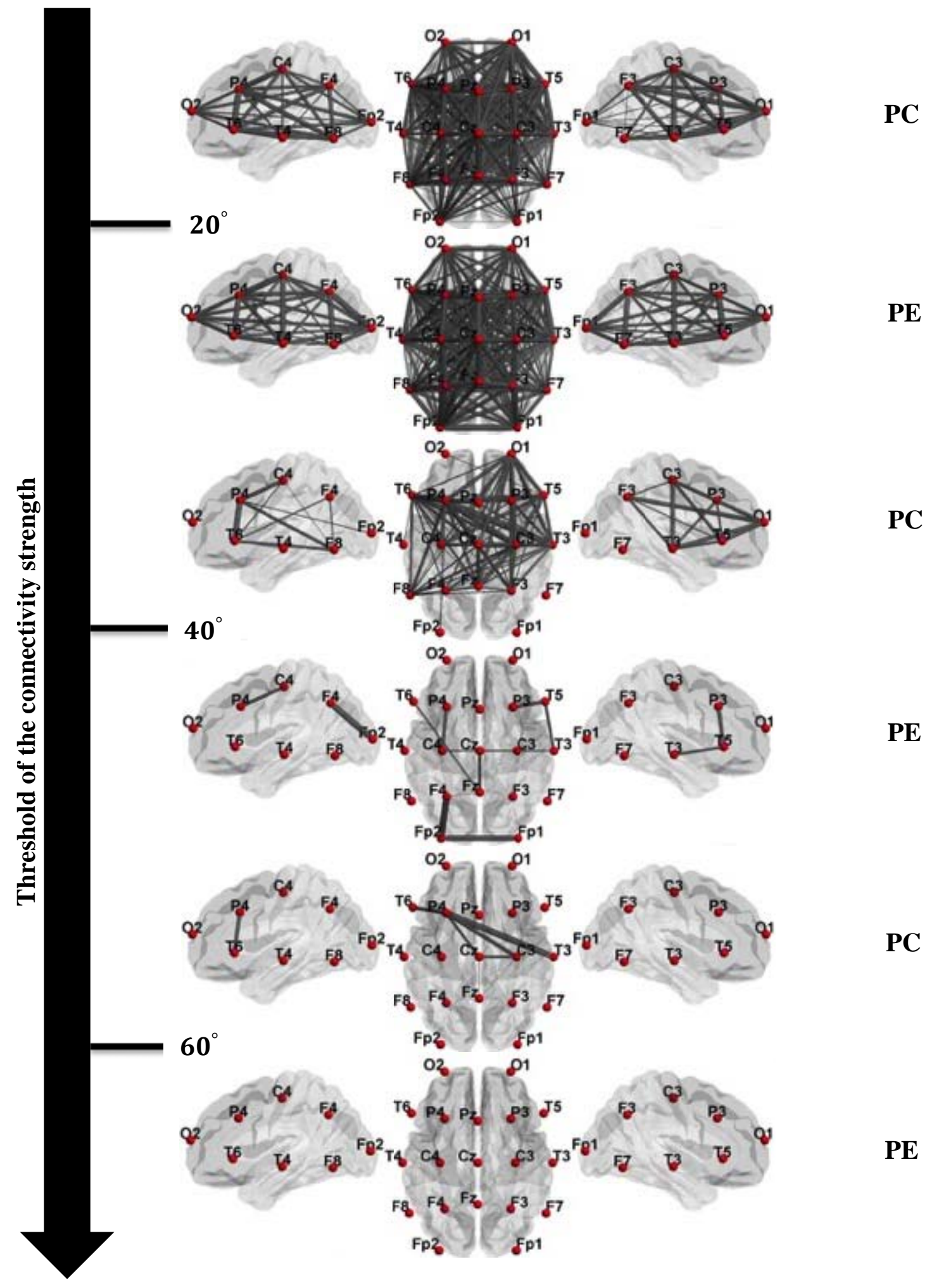

Figure 3.4: Graph representations of average of constructed FCNs over the pediatric control (PC) group and pediatric epilepsy (PE) group. Thickness of links (graph edges) shows the strength of connectivity among electrode pairs. 
However, to observe the network measure changes with different threshold, a set of thresholds were applied on the FCN's and the statistical significance of the groups' difference of graph theoretical measures were computed and plotted in Figure 3.5.

Table 3.3: First and second moment statistics for feature vectors across group of subjects.

\begin{tabular}{l|lll} 
FEATURE & Control Group & Epilepsy Group & Student t-test \\
\hline ld & $51.9 \pm 13.23$ & $58.86 \pm 20.88$ & $\mathrm{p}=0.45$ \\
adn & $934.29 \pm 238.1$ & $1059.5 \pm 375.8$ & $\mathrm{p}=0.46$ \\
deg & $934.29 \pm 238.16$ & $1059 \pm 375.82$ & $\mathrm{p}=0.45$ \\
adnn & $20.21 \pm 1.25$ & $19.48 \pm 0.79$ & $\mathrm{p}=0.18$ \\
acc & $0.001 \pm 0.0005$ & $0.002 \pm 0.005$ & $\mathrm{p}=0.5$ \\
abc & $0 \pm 0$ & $0 \pm 0$ & $\mathrm{p}=\mathrm{NaN}$ \\
gcc & $1.05 \pm 0.001$ & $1.05 \pm 0.002$ & $\mathrm{p}=0.52$ \\
rcc & $51.9 \pm 13.23$ & $59.33 \pm 19.48$ & $\mathrm{p}=0.4$ \\
smg & $3.53 \times 10^{8} \pm 1.5 \times 10^{8}$ & $4.5 \times 10^{8} \pm 1.8 \times 10^{8}$ & $\mathrm{p}=0.18$ \\
acg & $741.64 \pm 234.29$ & $923.32 \pm 342.77$ & $\mathrm{p}=0.25$ \\
eng & $1964 \pm 405.1$ & $2142 \pm 751.4$ & $\mathrm{p}=0.5$ \\
\hline
\end{tabular}

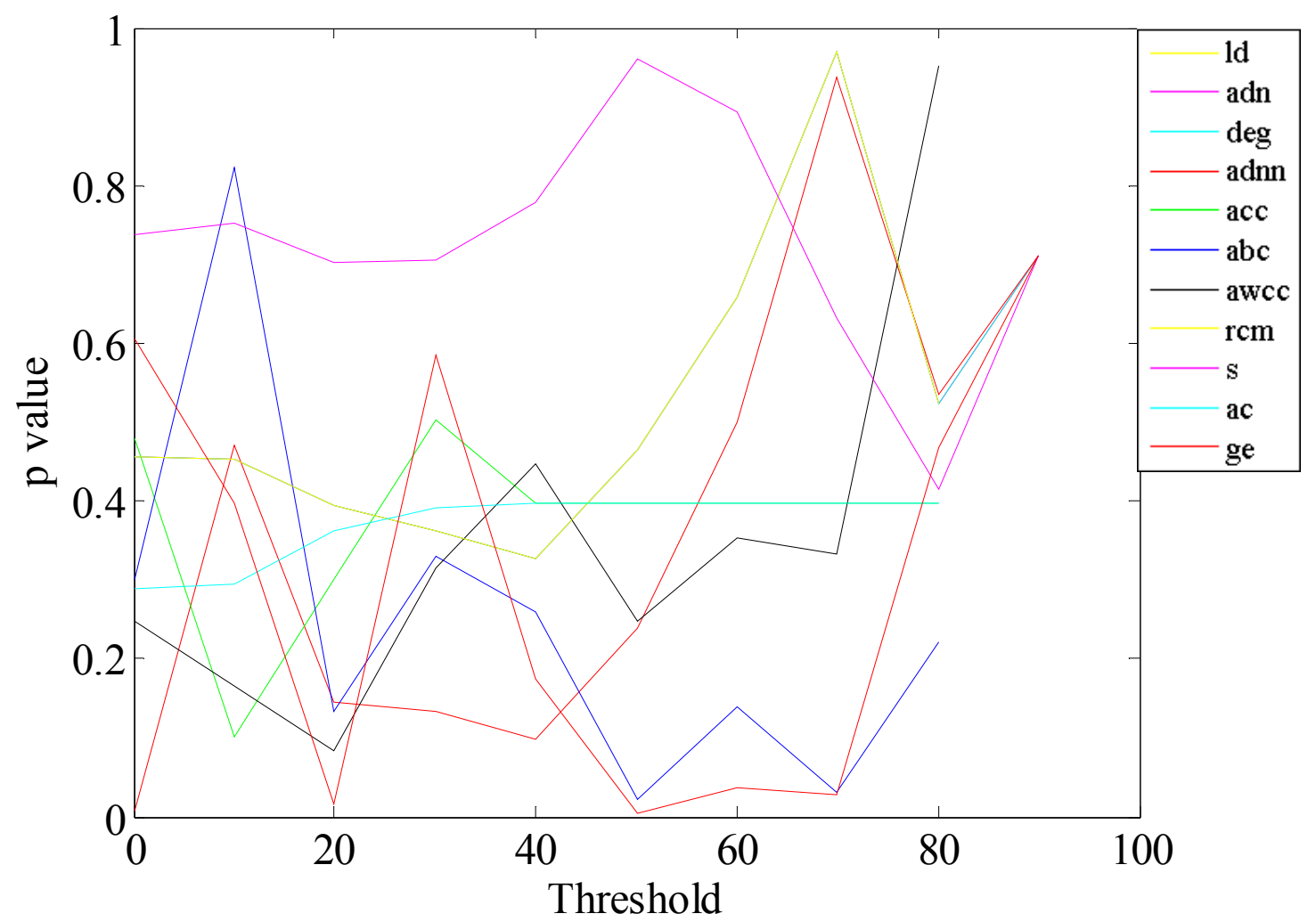

Figure 3.5: Statistical significance of the group difference for network graph measures as the function of threshold. 
The extracted feature vectors for the subjects were provided as input to the $K$-means clustering step to categorize subjects into one of the two groups. The classification technique developed using graph features from FCNs assumed no prior diagnostic knowledge rendering the technique completely data-driven. To count for the possible variation in the classification results due to the initialization of K-means, the algorithm was repeated over fifty times, however no variation was observed in the classification decision. Table 3.4 summarizes the results of the classification performed on the group of subjects with the accuracy, sensitivity and specificity to demonstrate the effectiveness of the technique. The confusion matrix provided in Table 3.4 demonstrates that the developed technique differentiates epileptic and healthy subjects with $87.5 \%$ accuracy considering no prior knowledge on the diagnosis.

Table 3.4: Clustering Results with no prior knowledge on diagnosis

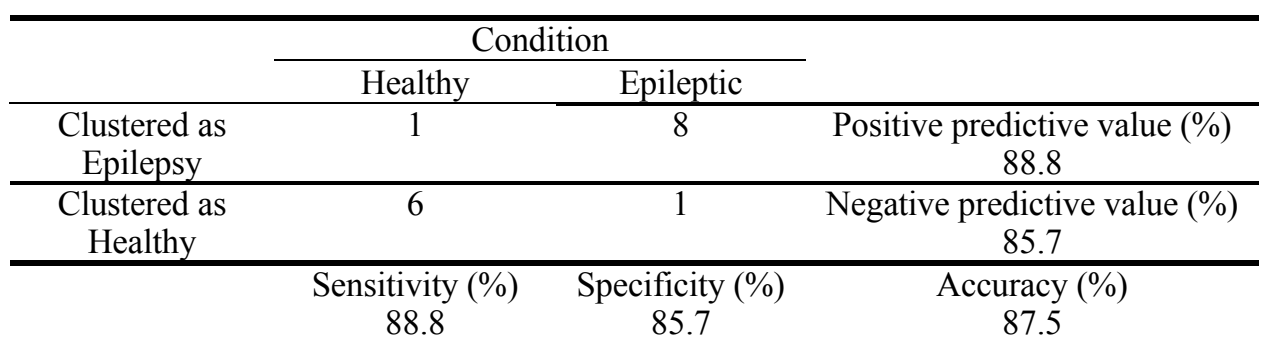

With the inclusion of the rater opinions on the diagnosis for each subject, SFS could find the subsets of features which outperform others in MSE sense. To evaluate the algorithm with the inclusion of the rater opinion, the Leave One Out Cross Validation (LOOCV) were done across all subjects. The accuracy in this case improved significantly to $96.87 \%$. 


\subsection{Algorithm II: GMM-based Approach}

The main contribution of the study is in introducing a novel approach based on purely machine learning techniques to facilitate the screening process of potential epileptic patients. The emphasis of developing the new decision support system for pediatric epilepsy diagnosis was placed on the accurate diagnosis without a priori knowledge. The probabilistic decision on the subject diagnosis makes the system more applicable in clinical environments. An overview of the proposed decision support system for computer aided diagnosis of pediatric epilepsy is as shown in Figure 3.6. The Algorithm starts with the segmentation of multichannel EEG recordings by applying a moving window (w) with overlap. Functional connectivity networks are constructed and mapped into a corresponding graph for each window. Extraction of graph theoretical based features $\left(\mathrm{u}_{-} \mathrm{w}\right)$ is then followed by a decision making process which uses a probabilistic approach to determine whether a patient is epileptic or not.

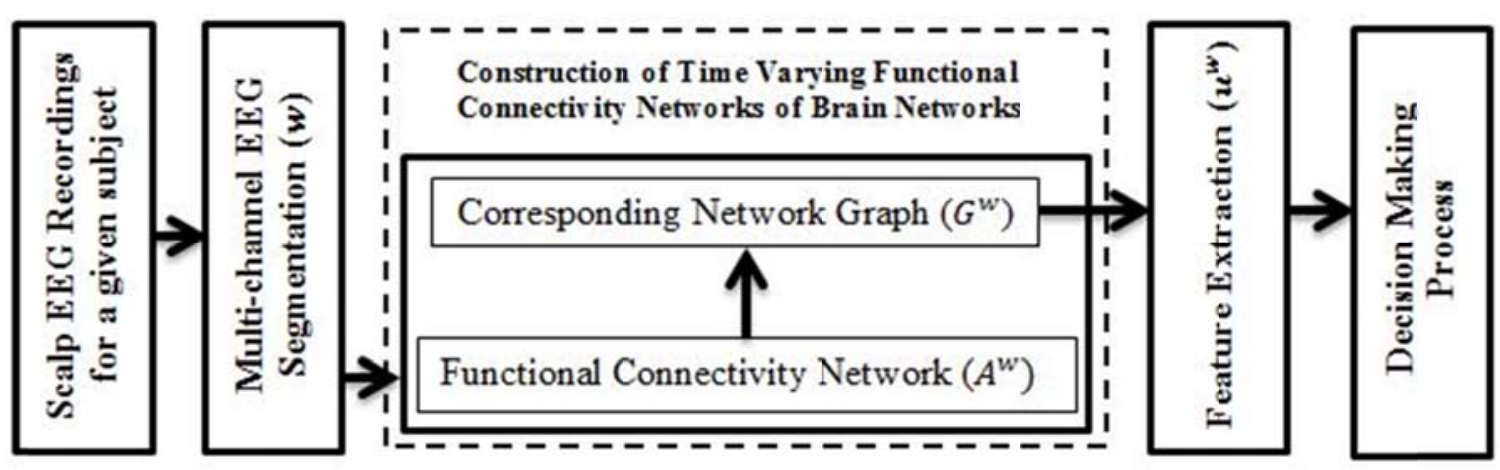

Figure 3.6: Flowchart of the decision support system

\subsubsection{Subjects and Data}

Scalp EEG recordings from 18 pediatric patients (7 pediatric control (PC) group and 11 pediatric epilepsy (PE) patients) were included in this study. The study was approved by the Institutional Review Board (Protocol number: IRB-052708-03) and consent forms 
were provided to the patients or legal representatives. The scalp Recordings were performed using XLTEK Neuroworks Ver. 3.0.5 equipment following 10-20 electrode placement system (referential montage) and sampled, indistinctly for PC and PE subjects, at varying frequencies of $200 \mathrm{~Hz}, 500 \mathrm{~Hz}$ and $512 \mathrm{~Hz}$. Segments free of artifacts, 4.2 seconds to 90 seconds long, from all EEG recordings were initially selected. Care was given to the initial selection of EEG segments such that they contained no seizure activity to prevent any bias. The inclusion of interictal spikes, as a representation of cortical irritability, is based on the fact that they could be observed in normal control subjects without being considered as epilepsy segments (Cabrerizo, Ayala et al. 2011). Due to non-stationary characteristics of long recordings of EEG signal (Wu and Gotman 1998; Cabrerizo, Ayala et al. 2011), and for an unbiased evaluation of the study results, initial intervals were broken into segments with equal length by applying a moving window with the length of 9 seconds and 4.5 seconds overlap. The choice of 9-second window length was based on the EEG segmentation strategy using the energy function calculated using the Teager's algorithm (Kaiser 1990; Wu and Gotman 1998). An overview of demographic characteristics of study subjects is given in Table 3.5.

Table 3.5: Demographic characteristics of subjects

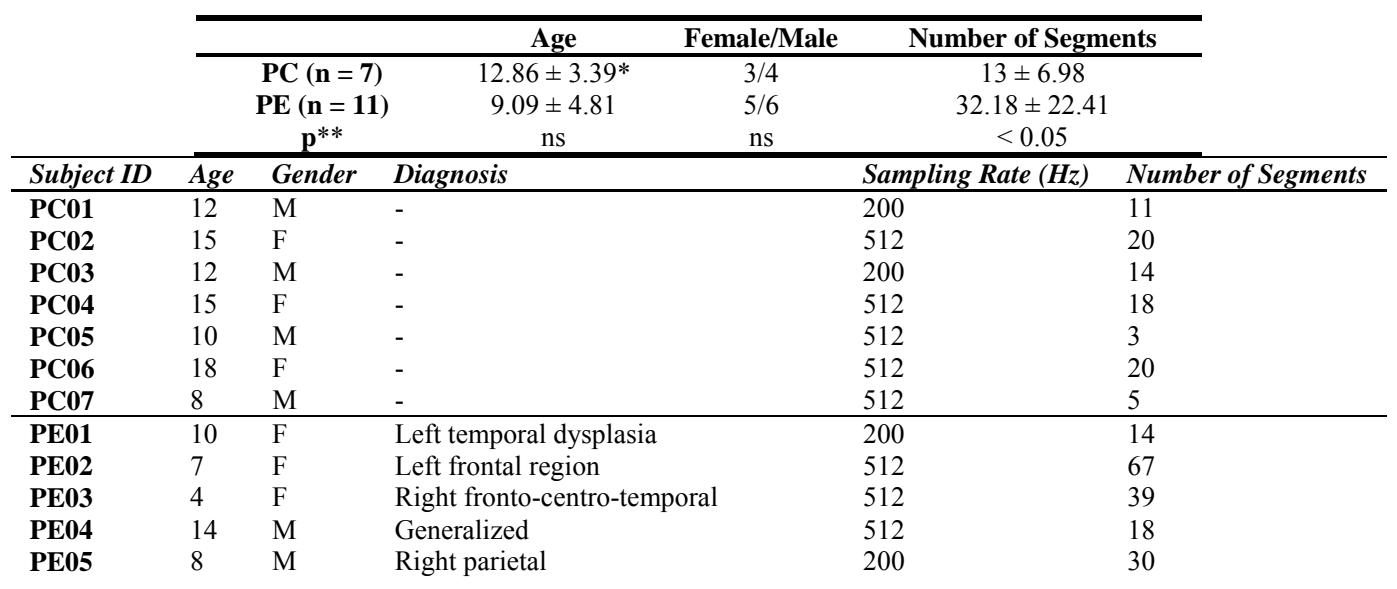




\begin{tabular}{|c|c|c|c|c|c|}
\hline PE06 & 7 & M & Left frontal pole, posterior frontal lobe & 512 & 77 \\
\hline PE07 & 15 & $\mathrm{~F}$ & Left and right frontal & 500 & 40 \\
\hline PE08 & 4 & M & Right fronto-centro-temporal & 512 & 25 \\
\hline PE09 & 2 & $\mathrm{~F}$ & Left temporal (posterior) & 512 & 25 \\
\hline PE10 & 14 & M & Generalized & 512 & 6 \\
\hline PE11 & 15 & M & Generalized & 512 & 13 \\
\hline & $\begin{array}{l}\text { *Dat: } \\
* * \text { Stu } \\
\text { Fishe } \\
\text { PC }= \\
\text { M: M } \\
\text { ns: nc }\end{array}$ & exa & $\begin{array}{l}\text { mean } \pm \text { S.D. where applicable, } \\
\text { th statistical significance threshold of } 0 \\
\text { vas used to test for gender. } \\
\text { trol; PE = Pediatric Epilepsy; }\end{array}$ & wa & e and number of segments. \\
\hline
\end{tabular}

\subsubsection{Functional Connectivity Network Construction}

The aim of functional connectivity of brain networks construction is to determine the existing pattern of functional association among hypothetically isolated channels and the causality relationship between anatomical brain regions. Emphasis is placed on the connection topology in the functional layer rather than on discovery of the existence of structural medium among cortical regions. Identifying the frequency based pattern of information propagation between brain regions to study the epileptic discharges has been recently investigated (Cabrerizo, Ayala et al. 2011). This study focuses on exploring the relationship among brain regions based on the time-domain electrical activities recorded using multi-channel scalp EEG. Time-domain characteristics of seizures and epileptic form discharges (Wu and Gotman 1998) enforce the computer aided diagnosis systems to look over short segments of EEG. Studying the dynamics of the neural networks within the cerebral cortex at higher time resolution increases the accuracy of interictal spike detection and 3-D source localization of seizures (Cabrerizo, Ayala et al. 2011; Wilke, Worrell et al. 2011). The proposed solution explores FCNs using multi-channel EEG segments (9 seconds long ( $\mathrm{Wu}$ and Gotman 1998)), which through a geometrical approach, the time-varying patterns of FCNs are assessed. FCNs are calculated in the form of a two dimensional array called adjacency matrix, $A^{w}{ }_{\text {und }}$, as defined in equation 
(3.6), where $w$ defines a segment of multi-channel EEG data and with subscript und denoting the undirected from. Elements of adjacency matrix are the pairwise strength of connectivity among scalp EEG recording channels for the segment $w$.

$$
\begin{gathered}
A^{w}{ }_{\text {und }}=\left[\theta_{i j}{ }^{w}\right]_{m \times m} \quad i, j=1, \ldots, m \\
\theta_{i j}{ }^{w}=\pi-\cos ^{-1}\left(\frac{\sum_{n=1}^{N} x_{i}[n] x_{j}[n]}{\sqrt{\sum_{n=1}^{N} x_{i}{ }^{2}[n]} \sqrt{\sum_{n=1}^{N} x_{j}^{2}[n]}}\right) \quad i, j=1, \ldots, m
\end{gathered}
$$

Undirected FCNs assume no causality in calculating the connectivity strength, which establishes a symmetric adjacency matrix. Following the symmetric property of undirected FCN, a geometric distance as shown in equation (3.7) is used to calculate the pairwise connectivity strength. The proposed geometric distance is a modification of the cosine similarity metric.

Each $\theta_{i j}{ }^{w}$ value represents the pairwise connectivity strength between electrodes $i$ and $j$ for the corresponding window $w$ with their electrical activity recordings denoted as $x_{i}[n]$ and $x_{j}[n]$, respectively. The value of $n$ represents the discrete time sample number which ranges from 1 to $N$ where $N$ is the length of window $w$, and $m$ is the number of electrodes considered for constructing the FCNs. The angular value of $\theta_{i j}{ }^{w}$ of 0 radian specifies the highest connectivity strength which is most likely the case when calculating

the distance among an electrode and itself, and a value of $\frac{\pi}{2}$ radian shows that the respective pair of electrode recordings is orthogonal and corresponds to the maximum distance (i.e., lowest connectivity strength).

\subsubsection{Graph Mapping of Brain FCN's and Feature Extraction}

Graph theory is a well-established and rich source of benchmarks for studying functional 
connection as well as anatomical connections in the brain (Van Wijk, Stam et al. 2010). Directed FCNs $\left(A_{\text {dir }}^{w}\right)$ and undirected FCNs $\left(A^{w}\right.$ und $)$ in terms of connectivity strength among pair of scalp EEG channels recordings could be mathematically represented and studied in the forms of directed and undirected network graphs (Eddin, Wang et al. 2014). Graph $G^{w}$, for a given segment $w$, is comprised of a set of vertices, $V^{w}$, and a set of edges, $E^{w}$. In the context of FCNs, vertices are the scalp EEG electrodes and their location in the graph can be determined from the coordinate of the electrode position in the 10-20 electrode placement system space. Similarly, the graph edges are the hypothetical functional connection among the vertices and the connection strengths calculated as in equations (3.6) and (3.7) define the weights of these edges. In mapping these FCNs, it is hypothesized that the small world network model of brain function could be altered by epilepsy (Ponten, Bartolomei et al. 2007; Ponten, Douw et al. 2009; Sargolzaei, Cabrerizo et al. 2013). These hypothesized alterations could be used to consequently discriminate a network graph corresponding to a patient with epilepsy from that of a control group. Network graph $G^{w}$ based statistics (Table 3.6) (Bounova and de Weck 2012) are calculated for each segment $w$ to form the feature vector $u^{w}$ as shown in equation (3.8):

$$
u^{w}=\left[\begin{array}{lllllll}
l d g & \text { acc } & \text { gcc } & \text { rcc } & \text { smg } & \text { acg } & \text { eng }
\end{array}\right]^{T}
$$

Table 3.6: Graph theoretical features of functional connectivity networks

\begin{tabular}{lll}
\hline Feature & Feature Description & Feature calculation \\
\hline $\operatorname{ldg}$ & Link density of the graph & $(2 \times n e) /(n n \times(n n-1))$ \\
acc & Average of closeness centrality & $(1 / n n) \sum_{n n}($ sum of reciprocal distances from a node to all other nodes $)$ \\
$\operatorname{gcc}$ & Graph clustering coefficient & $(3 \times$ number of triangles $) /($ number of connected triples of nodes $)$ \\
rcc & Rich club coefficient & $\left(n e_{-} k\right) /\left(n n_{-} k \times\left(n n_{k}-1\right)\right)$ \\
smg & S-metric of graph & Sum of the nodal degree products for every edge. \\
acg & Algebraic connectivity of graph & Second smallest eigenvalue of the Laplacian of adjacency matrix.
\end{tabular}


$n e$ : Number of graph edges; $n n$ : number of graph nodes;

$n n \_k$ : Number of nodes with degree larger than $k ; n e_{-} k$ : number of edges among nodes with degree larger than $k$.

\subsubsection{GMM-based Decision Making Process}

As the multichannel scalp EEG is broken into segments $(w)$ and analyzed to generate the feature vectors $\left(u^{w}\right)$, when using a training set with annotated EEG segments (epileptic or non-epileptic), a classifier could be trained to make a decision on a newly recorded EEG segment in the testing phase. However in the absence of training data and/or a priori diagnosis, machine learning techniques could be instead incorporated to make an optimized decision on the nature of segment whether it belongs to the PE or PC group. One of the merits of the current study is in the ability to identify epileptic EEG's from non-epileptic EEG when no prior knowledge is at hand. The proposed system as designed consists of two phases of decision making. Figure 3.7 shows the steps in making a decision. Phase I assigns labels to multichannel scalp EEG segments based on the Gaussian Mixture Modelling of graph theoretical based features; Phase II assigns labels (epileptic or non-epileptic) to the subjects by assigning a probability in the likelihood of belonging in one of the two groups. 


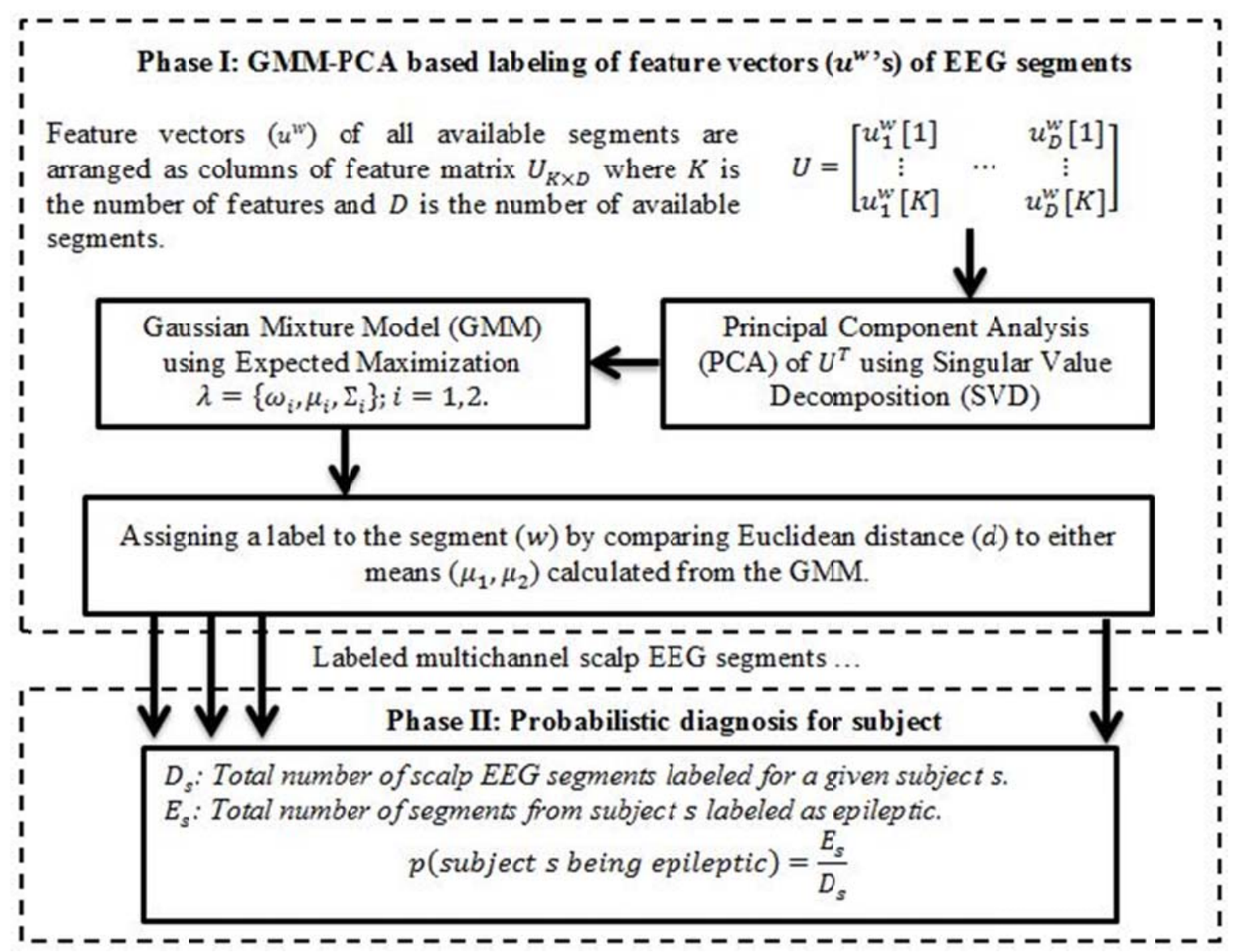

Figure 3.7: Flowchart of the two-phase decision making process.

The input to the decision making block is a feature matrix, $U_{K \times D}$, whose columns are the feature vectors corresponding to the segments, where $K$ is the number of features and $D$ is the total number of segments. This feature matrix is then subjected to the principal component analysis (PCA) using singular value decomposition (SVD) (Lagerlund, Sharbrough et al. 1997) This step is to map the covariance of the data into a new coordinate whose bases express the most variance. The output of PCA using the first principal component, $x_{1 \times D}$, is considered as the probability distribution of graph theoretical features $\left(u^{w}\right)$ across all segments. $x_{1 \times D}$ is then modeled parametrically using a Gaussian Mixture Model (GMM) using the two mixtures $\lambda=\left\{\omega_{i}, \mu_{i}, \Sigma_{i}\right\} ; i=1,2$ as 
shown in equation (3.9).

$$
p(x \mid \lambda)=\sum_{i=1}^{2} \omega_{i} g\left(x \mid \mu_{i}, \Sigma_{i}\right)
$$

where $\omega_{i}$ elements are the mixture weights, and $g\left(x \mid \mu_{i}, \Sigma_{i}\right)$ represents the Gaussian densities calculated from $D$-variate Gaussian distribution with parameters $\mu_{i}$ and $\Sigma_{i}$ $(i=1,2)$ as their respective means and covariance matrices (Lagerlund, Sharbrough et al. 1997; Ziaei, Sangwan et al. 2012; Xia, Wang et al. 2013). Parameters of the model were estimated through maximum likelihood (ML) estimation method and EEG segments are then labelled based on their closeness to the either estimated means.

The decision support system developed in the current study serves as an auxiliary tool in clinical settings. Therefore providing the neurologists with a probability of the risk factor for a given subject is more preferable rather than logical decisions (epileptic vs. nonepileptic). The number of segments labeled as epileptic for a specific subject is used in phase II of the decision making process to assign a probability as shown in equation (3.10) on the evidence of these segments' labeling.

$$
p(\text { subject is epileptic })=\frac{E_{S}}{D_{s}}
$$

where $E_{s}$ is the number of segments from the given subject being labeled as epileptic out of total number of segments $D_{s}$ for the corresponding subject.

\subsubsection{Implementation Results}

Figure 3.8 shows the constructed undirected FCNs averaged for both pediatric epilepsy (PE) and pediatric control (PC) groups with their corresponding bar plots of connectivity distances (degrees) and under different degrees of strengths. The symmetric property of the adjacency matrices is due to the nature of the constructed FCNs which do not take into 
consideration the possible causality among brain cortical region. The change in the pattern of functional associations is observed by increasing the distances among some brain regions such as cz-o1 and cz-o2 pairs. A two-sided two sample student t-test was conducted to assess whether there is a significant difference between the undirected mean FCNs of the PC group and the PE group. The test results indicated that there is a statistically significant difference $(t(340)=-9.89, p<0.0001)$ between the mean FCNs of the PC group $\left(\mu_{P C}=44.56^{\circ}, \sigma_{P C}=13.75^{\circ}\right)$ and the PE group $\left(\mu_{P C}=\right.$ $\left.57.74^{\circ}, \sigma_{P E}=10.70^{\circ}\right)$

Furthermore, we performed a two-sample student $t$ test to inspect the existence of a statistically significant difference among each connection between source electrode and target electrode (index of electrode pair in Figure 3.8 (b) and (d)) across the pediatric control and pediatric epilepsy group. Results are visualized in Figure 3.8 (e). Existence of statistically significant difference $(p<0.00001$ Bonferroni adjusted for multiple comparisons) in the connection strength (degree) of a specific source-target pair of electrodes is shown as a black box unit; insignificant difference in the connection strength is shown as a white box unit. As the large number of black boxes in Figure 3 (e) states, the brain acts as a complex network and epilepsy affects more than single connection. However this needs to be more investigated on a subject basis as the type of epilepsy (focal or generalized) could vary from subject to subject. 


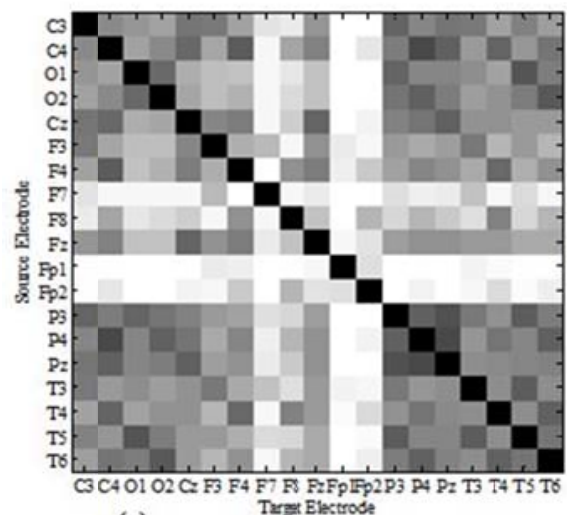

(a)

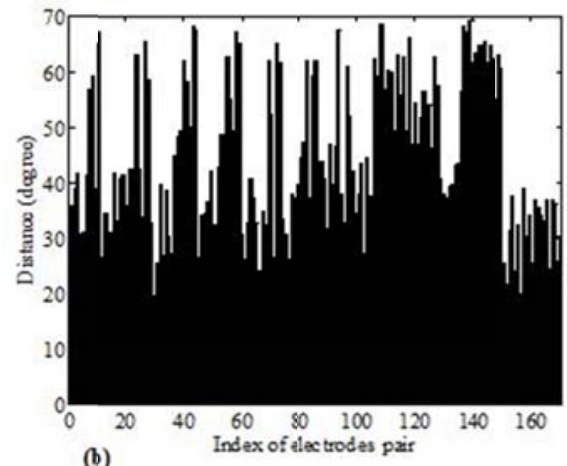

(b)

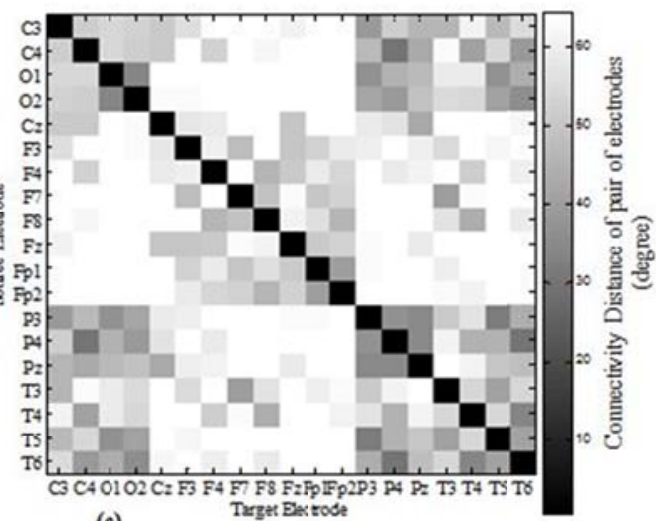

(c)

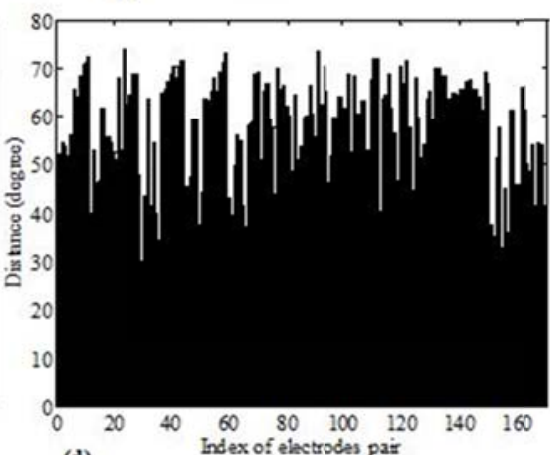

(d)

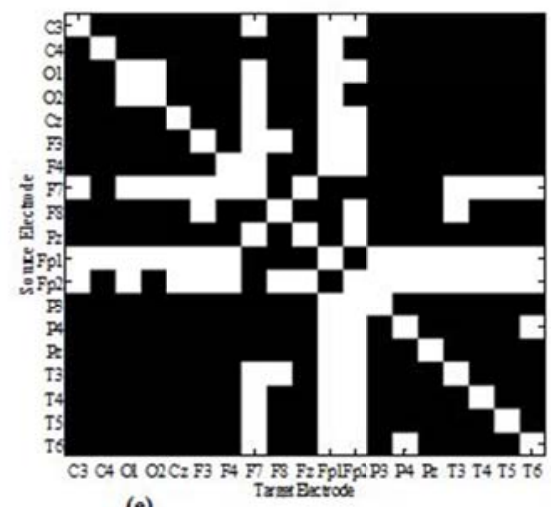

$\square<0.00001$

(e)

Figure 3.8: Visualization of constructed undirected FCNs.

Adjacency matrices shown in Figure 3.8 are mapped into the graph representations shown in Figure 3.9 showing graph representations of average of constructed FCNs over the (a) pediatric control (PC) group and (b) pediatric epilepsy (PE) group. Thickness of links (graph edges) shows the strength of connectivity among electrode pairs. Analysis of the 
single connections of the graphs is beyond the scope of this study; however the reduction in the pattern of inter-connections among isolated brain regions is visually observable by comparing the two graphs; Table 3.7 provides the statistics of the features extracted for both graphs and tests for the differences among PC and PE groups.

Table3.7: First and second statistics plus statistical analysis of feature vectors.

\begin{tabular}{lllc}
\hline Feature & PC & PE & p \\
\hline Idg & $44.56 \pm 7.77 * *$ & $57.74 \pm 8.44$ & $<0.0001$ \\
$\boldsymbol{a c c}$ & $0.0014 \pm 0.0003$ & $0.0010 \pm 0.0003$ & $<0.0001$ \\
$\boldsymbol{g c c}$ & $1.18 \pm 0.006$ & $1.18 \pm 0.005$ & $n s$ \\
rcc & $44.56 \pm 7.77$ & $57.74 \pm 8.44$ & $<0.0001$ \\
$\boldsymbol{s m g}$ & $2.38 \pm 0.81$ & $3.98 \pm 1.04$ & $<0.0001$ \\
$\boldsymbol{a c g}$ & $659.76 \pm 135.45$ & $913.24 \pm 169.46$ & $<0.0001$ \\
$\boldsymbol{e n g}$ & $1.68 \pm 0.26$ & $2.11 \pm 0.28$ & $<0.0001$ \\
\hline \multicolumn{5}{c}{} & $*$ Two-way Student t test is used for test the difference between PC and PE groups. \\
& Statistical significant level of 0.01 is considered for p-value.
\end{tabular}

We also performed a connection density assessment on left and right hemispheres and the interconnection among the hemispheres across subjects. Density of connections on each hemisphere were calculated by counting the number of edges with the weight angle less than or equal to $45^{\circ}$ (mid-point in connectivity strength). The results summarized in Table 3.8, show alterations in the wiring pattern of the brain functional network caused by epilepsy. This alteration is in the form of reduced density of connectivity in both left and right hemisphere as well as inter-hemispheric connectivity. 


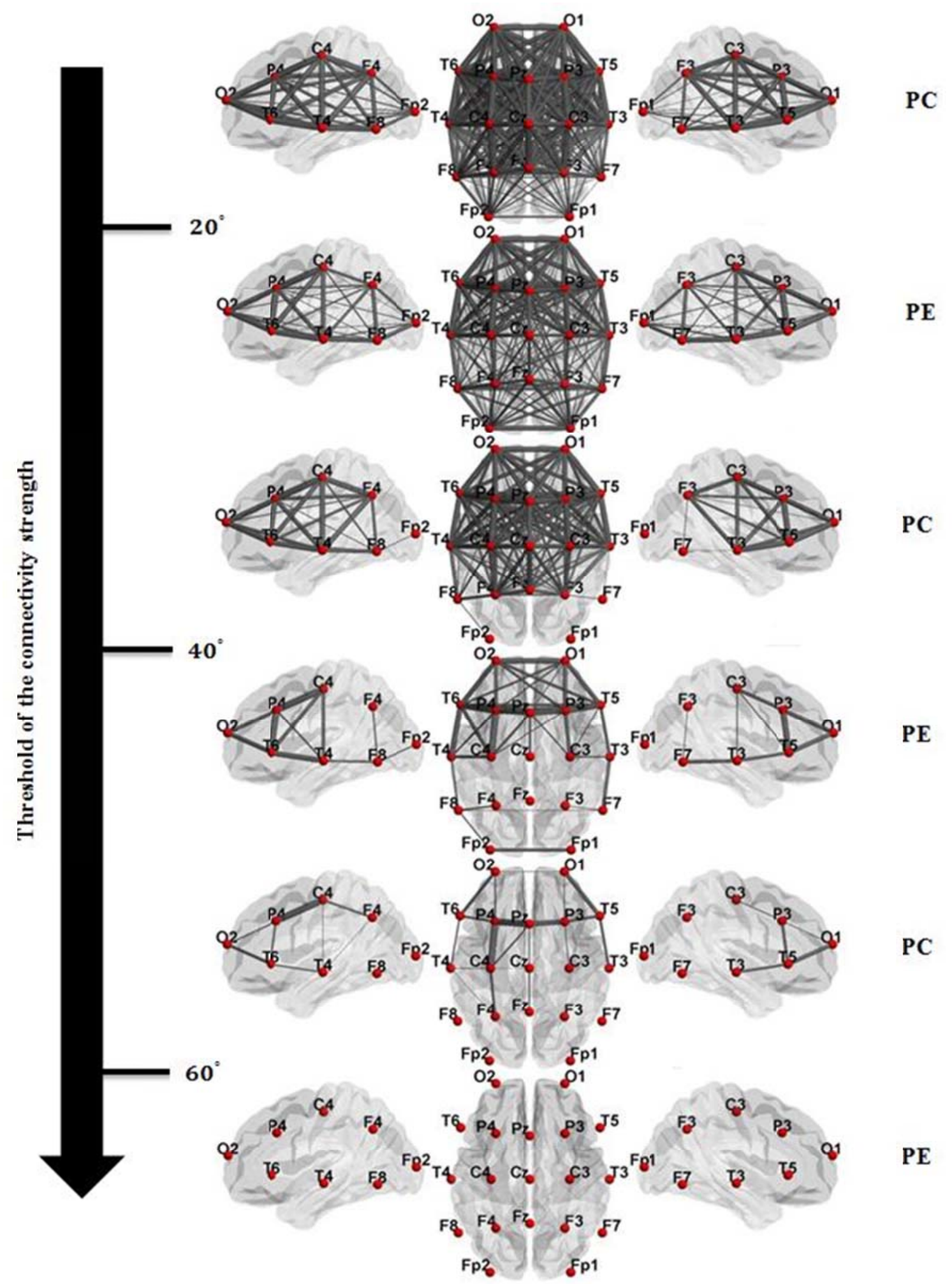

Figure 3.9: Graph representations of average of constructed FCNs. 
Table 3.8: Connectivity strength for left hemisphere, right hemisphere and interhemisphere

\begin{tabular}{|c|c|c|c|c|c|c|c|}
\hline & \multicolumn{4}{|c|}{ Hemisphere Connection } & \multicolumn{3}{|c|}{ Hemisphere Connection } \\
\hline & Right & Left & $\begin{array}{l}\text { Inter- } \\
\text { hemisphere }\end{array}$ & & Right & Left & $\begin{array}{l}\text { Inter- } \\
\text { hemisphere }\end{array}$ \\
\hline PC01 & $10.82 \pm 2.6$ & $9.09 \pm 4.23$ & $18 \pm 11.4$ & PE01 & $10.64 \pm 4.41$ & $3.42 \pm 2.79$ & $10 \pm 7.1$ \\
\hline PC02 & $19.3 \pm 4.12$ & $19.55 \pm 4.16$ & $34.1 \pm 12.45$ & PE02 & $9 \pm 3.39$ & $7.75 \pm 2.3$ & $9.75 \pm 5.98$ \\
\hline PC03 & $20 \pm 1.62$ & $15.71 \pm 1.14$ & $33.42 \pm 5.62$ & PE03 & $11.87 \pm 4.93$ & $16.28 \pm 6.1$ & $26.13 \pm 15.4$ \\
\hline PC04 & $16 \pm 5.72$ & $15.5 \pm 4.74$ & $27 \pm 18.82$ & PE04 & $15.28 \pm 4.84$ & $13.11 \pm 7.5$ & $22.16 \pm 17.5$ \\
\hline PC05 & $24.67 \pm 1.5$ & $15 \pm 1.73$ & $33.33 \pm 16$ & PE05 & $9.6 \pm 6.5$ & $11.5 \pm 5.3$ & $14 \pm 16.2$ \\
\hline PC06 & $18.7 \pm 2.56$ & $15.2 \pm 3.67$ & $35.75 \pm 7.57$ & PE06 & $9.7 \pm 1.38$ & $8.5 \pm 1.7$ & $19.25 \pm 4.78$ \\
\hline PC07 & $13.8 \pm 3.96$ & $12.8 \pm 3.96$ & $27 \pm 11.62$ & PE07 & $9.85 \pm 1.72$ & $11.97 \pm 4.4$ & $17.15 \pm 9.1$ \\
\hline \multirow{5}{*}{$\begin{array}{l}\text { Pooled } \\
\text { statistics }\end{array}$} & $17.4 \pm 3.45$ & $15.42 \pm 3.64$ & $30.59 \pm 11.5$ & PE08 & $6.28 \pm 2.82$ & $7.7 \pm 5.73$ & $9.16 \pm 11.43$ \\
\hline & & & & PE09 & $14.36 \pm 3.36$ & $5.1 \pm 1.1$ & $4.64 \pm 1.87$ \\
\hline & & & & PE10 & $5.6 \pm 3$ & $7 \pm 4.7$ & $11.17 \pm 7.3$ \\
\hline & & & & PE11 & $14.15 \pm 1.72$ & $15.38 \pm 5$ & $34 \pm 7.85$ \\
\hline & & & & $\begin{array}{l}\text { oled } \\
\text { tistics }\end{array}$ & $10.32 \pm 3.19$ & $9.82 \pm 3.64$ & $15.97 \pm 8.77$ \\
\hline
\end{tabular}

For the evaluation purposes, no information was provided for multichannel EEG segments in terms of diagnosis (epileptic vs. non-epileptic) of the subject to whom the segments belong to. Results of labeling procedure using Gaussian mixture model (GMM) and the probability of the corresponding subject being epileptic are given in Table 3.9. The suggested decision making system based on GMM has shown the ability of detecting epileptic segments with an accuracy of $81.3 \%$ with $77.4 \%$ sensitivity and $96.7 \%$ specificity solely based on discovery of the hypothetical associations among cortical regions. The probability approach shows the power of the proposed algorithm in decision making based on the segment labeling and time-varying FCNs as examplified in subjects PE04 and PC04. Both subjects have segments labeled as the contrary group which the subject indeed belongs to; however the probability approach based on the time-varying FCNs identifies them correctly when the decision is made. The mis-identification of labels in subjects PE03 and PE11 could not be corrected by the suggested probability approach. 
This misidentification could be due to different factors such as the window size, number of segments required to accurately diagnose or the type of epilepsy which needs more investigation.

Table 3.9: Clustering results with no prior knowledge provided on diagnosis

\begin{tabular}{|c|c|c|c|c|c|}
\hline & \multicolumn{3}{|c|}{ Condition } & & \\
\hline & \multicolumn{2}{|l|}{ Healthy } & Epileptic & ptic & \\
\hline $\begin{array}{l}\text { Clustered as } \\
\text { Epilepsy }\end{array}$ & 0 & \multicolumn{2}{|c|}{9} & Positive & $\begin{array}{l}\text { predictive value (\%) } \\
100\end{array}$ \\
\hline \multirow[t]{21}{*}{$\begin{array}{c}\text { Clustered as } \\
\text { Healthy } \\
\end{array}$} & 7 & \multicolumn{2}{|c|}{2} & Negative & $\begin{array}{l}\text { e predictive value (\%) } \\
77.8\end{array}$ \\
\hline & $\begin{array}{c}\text { Sensitivity (\%) } \\
81.8\end{array}$ & \multicolumn{2}{|c|}{$\begin{array}{c}\text { Specificity (\%) } \\
100\end{array}$} & \multirow{2}{*}{\multicolumn{2}{|c|}{$\begin{array}{cc} & \text { Accuracy (\%) } \\
\text { bility (\%) } & 88.9\end{array}$}} \\
\hline & Subject ID & $E_{s}$ & $D_{s}$ & & \\
\hline & PC01 & 0 & 11 & 0 & \\
\hline & PC02 & 0 & 20 & 0 & \\
\hline & PC03 & 0 & 14 & 0 & \\
\hline & PC04 & 2 & 18 & 11 & \\
\hline & PC05 & 0 & 3 & 0 & \\
\hline & PC06 & 0 & 20 & 0 & \\
\hline & PC07 & 1 & 5 & 20 & \\
\hline & PE01 & 12 & 14 & 86 & \\
\hline & PE02 & 67 & 67 & 100 & \\
\hline & PE03 & 10 & 39 & 25 & \\
\hline & PE04 & 12 & 18 & 66 & \\
\hline & PE05 & 21 & 30 & 70 & \\
\hline & PE06 & 66 & 77 & 86 & \\
\hline & PE07 & 30 & 40 & 75 & \\
\hline & PE08 & 23 & 25 & 92 & \\
\hline & PE09 & 25 & 25 & 100 & \\
\hline & PE10 & 5 & 6 & 83 & \\
\hline & PE11 & 3 & 13 & 23 & \\
\hline
\end{tabular}

If the assumption of no a priori knowledge on the diagnosis were to be removed, a training set of multi-channel EEG segments could be considered as a tuning approach in the decision making process. A training set were composed including twenty randomly chosen multi-channel EEG segments from the total set of EEG segments and Support Vector Machine (SVM) with a linear kernel were trained to classify the segments. The testing set was then given to the system after self tuning and the results showed $100 \%$ accuracy in classification accuracy of the multi-channel EEG segments. 


\subsection{Conclusion}

Two new algorithms for FCN's construction were proposed and developed in this chapter. Graph theoretical analysis of the networks was utilized for clinical diagnosis of pediatric epilepsy. 


\section{CHAPTER 4}

\section{Temporal Analysis of FCNs in Epilepsy}

\subsection{Introduction}

This chapter investigates the evolution of functional brain connectivity networks during continuous interictal EEG recordings. Interictal EEG recordings take about 20-40 minutes, usually performed in an outpatient laboratory environment, to establish the presence of epilepsy as well as to serve for classifying the type of epilepsy (Focal or Generalized) (Pillai and Sperling 2006). Operation surgery to remove the epileptogenic brain tissue zone is currently the only possible cure for pharmaceutical-resistance patients; however epileptogenic foci localization is performed following invasive presurgical EEG recordings (Zwoliński, Roszkowski et al. 2010). This chapter of the dissertation emphasizes utilization of our proposed FCN construction technique to study the FCNs related to pre- inter- and post- stages of the evolution of interictal EEG recordings.

\subsection{Dataset}

The EEG data used for this chapter of the dissertation are part of a publicly accessible dataset (Zwoliński, Roszkowski et al. 2010). The dataset includes continuous interictal EEG recordings of severe epilepsy patients who had undergone resection surgery. All the recordings were resting state data and performed before the surgery using the international 10-20 system. The data were band-pass filtered for $0.5-70 \mathrm{~Hz}$ and notch filtered for $50 \mathrm{~Hz}$. Table 4.1 provides the demographic information for the subject studies included in this chapter from the complete dataset (Zwoliński, Roszkowski et al. 2010). Detailed information can be found in the database website (eeg.pl/epi). More detailed 
information on the diagnosis will be given in the subject-specific discussion of the chapter.

Table 4.1: Study subjects of Chapter 4

\begin{tabular}{|l|l|l|l|l|l|}
\hline ID & Subject* & $\begin{array}{l}\text { Ag } \\
\text { e }\end{array}$ & Sex & $\begin{array}{l}\text { Number of } \\
\text { Ictal Events** }\end{array}$ & Diagnosis \\
\hline P01 & CHIMIC & 9 & M & 3 & Temporal lobe epilepsy \\
\hline P02 & FRAANN & 17 & $\mathrm{~F}$ & 2 & Temporal lobe Epilepsy \\
\hline P03 & HRADAW & 12 & $\mathrm{M}$ & 4 & Generalized epilepsy \\
\hline P04 & JANPAT & 10 & $\mathrm{~F}$ & 4 & Temporal lobe epilepsy \\
\hline P05 & JANPRZ & 7 & $\mathrm{M}$ & 4 & Severe form of epilepsy \\
\hline P06 & JATKAM & 14 & $\mathrm{~F}$ & 7 & Temporal lobe epilepsy \\
\hline P07 & GILPAU & 14 & $\mathrm{~F}$ & 5 & Frontal lobe epilepsy \\
\hline P08 & KOSPAW & 16 & $\mathrm{M}$ & 3 & Severe epilepsy and Mild cognitive impairment \\
\hline P09 & KROMIC & 16 & $\mathrm{M}$ & 5 & Severe epilepsy and Mild cognitive impairment \\
\hline P10 & MARPAW & 16 & $\mathrm{M}$ & 4 & Temporal lobe epilepsy \\
\hline P11 & MATPAW & 15 & $\mathrm{M}$ & 3 & Generalized seizures \\
\hline P12 & NIZMAR & 12 & $\mathrm{M}$ & 3 & Motor lobe seizures \\
\hline P13 & NOWJON & 15 & $\mathrm{M}$ & 3 & Temporal lobe epilepsy \\
\hline P14 & SIEGRZ & 18 & $\mathrm{M}$ & 4 & Mesial temporal lobe epilepsy \\
\hline P15 & SNOKAC & 3 & $\mathrm{M}$ & 6 & Severe form of epilepsy \\
\hline
\end{tabular}

*Subject reference to the original database.

**Number of used ictal events may be less than the number of interictal events in the original database.

\subsection{Methods}

\subsubsection{Ictal Events Segmentation}

Epileptogenic waveforms marked by the epileptologist (Zwoliński, Roszkowski et al. 2010) were used to segment EEG recordings to three different phases of the event by moving a window with the length of the event over the recordings in time:

- pre: The window is located right before the initiation of the interictal event.

- inter: The window includes the interictal event.

- post: The window is fully outside of the interictal event.

\subsubsection{Functional Connectivity Networks Construction}

Functional connectivity networks (FCNs) were all constructed following the methodology introduced in Chapter 3. 


\subsubsection{Brain FCNs Binarization}

Undirected weighted connectivity networks were then thresholded to unweight the network and facilitate the network comparison for graph theoretical analysis of interictal event evolution. The thresholding scheme was based on graph density and fully explained in (Eddin, Wang et al. 2014). Thresholding based on graph density results in networks with the same number of nodes and same number of edges but the edges are shifted highlighting the changes in the wiring scheme of network over time.

\subsubsection{Graph Theory Analysis of FCNs}

Visual inspections of the alterations of FCNs over different interictal events were performed to investigate the alterations of the progression of events as well as hypothesized similarities among intra-subject interictal events. Clustering coefficient, global efficiency (characteristic path length) and small world index of the networks have been calculated for quantitative analysis of brain FCNs in the presence of interictal events. Brain Connectivity Toolbox (Rubinov and Sporns 2010), MIT MATLAB tools for network analysis (Bounova and de Weck 2012) and SBEToolbox (Konganti, Wang et al. 2013) were utilized to perform the network analysis and measurements.

\subsection{Results}

Results of this chapter are given in subject-specific domain as well as group analysis of three important characteristics (Clustering Coefficient, Global Efficiency and Small World index) of the brain networks at three stages of interictal recordings. The patient's clinical and diagnosis information given here are from the original dataset (Zwoliński, Roszkowski et al. 2010). 


\subsubsection{Subject-Specific Analysis}

All the FCNs are the density-based thresholded maps and the overlaid circle in average maps represents the approximate location of the revealed pathological mass found in the MRI.

P01: Temporal lobe epilepsy; seizures with simple partial onset evolving to partial complex (motor automatisms); Side and focus of discharges hard to determine. MRI revealed a mass in right temporal origin (hippocampal region). Seizure free after right hippocampal complex resection. According to the medical evaluation of the subject, interictal EEG showed generalizing sharp spikes, making side and focus localization process of discharges hard. FCNs in Figure 4.1 are showing the similarities among the initiation of the interictal events across different epochs. MRI revealed the existence of a mass in right temporal area. The average FCNs is showing the right temporal electrodes presence as one of the active common sub-network in all epochs. Network measurements verify the similarity observed in the pattern of the FCN's among epoch 2 and 3, which are in close proximity of each other in the time domain. Figure 4.2 shows the graph theory measurements corresponding to pre- inter- post- ictal stages, respectively for different epochs for P01 


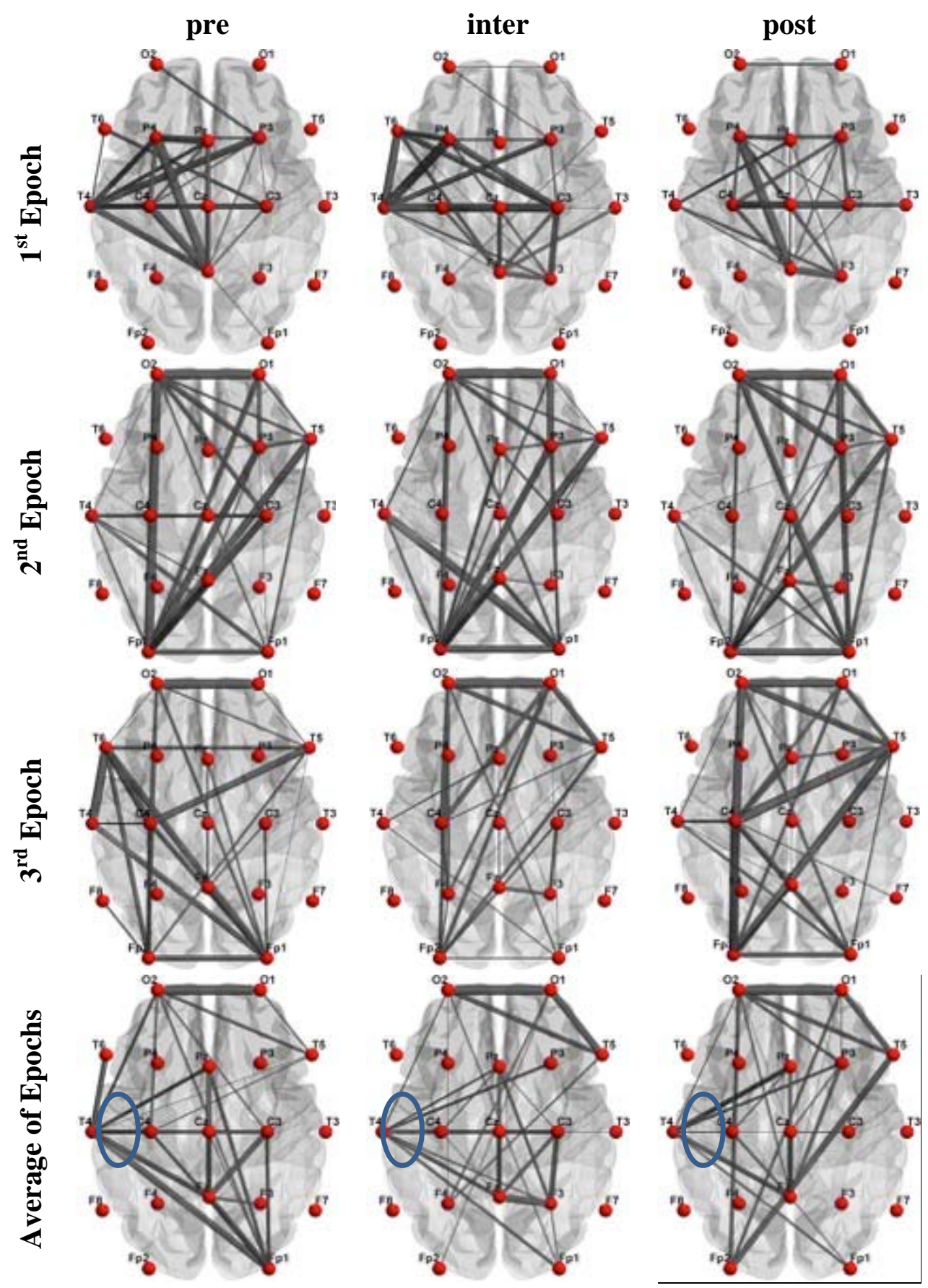

Figure 4.1: Functional connectivity networks corresponding to pre- inter- post- ictal stages for different epochs across for P01. 

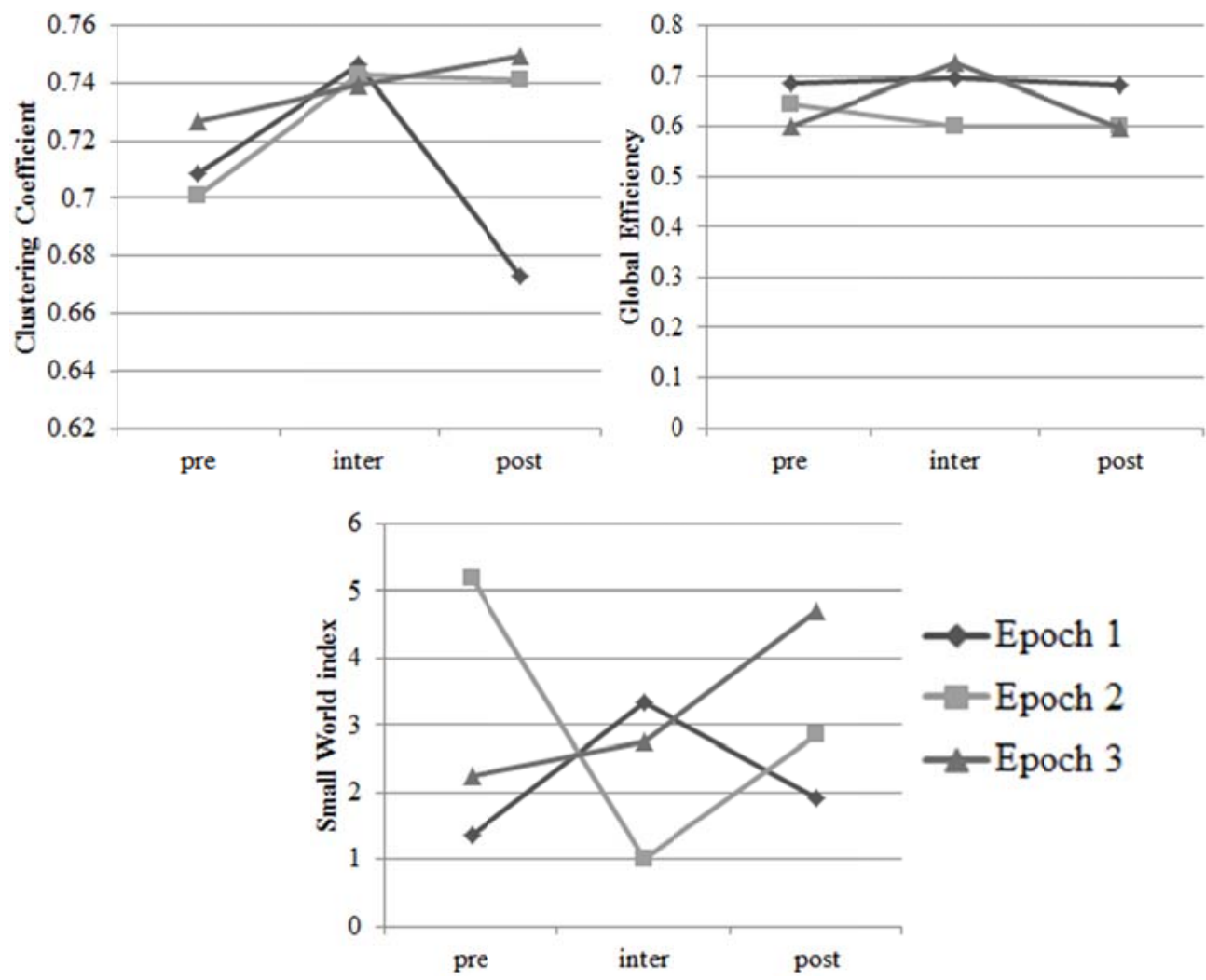

Figure 4.2: Graph Theory Measurements corresponding to pre- inter- post- ictal stages for different epochs across for P01.

P02: Severe form of refractory temporal epilepsy. Primarily as pseudo-absences with motionless stare and salivation, then later evolving to generalized tonic-clonic. Some repetitive synchronous spiking within right fronto-temporal region. MRI revealed a mass within the right hippocampus. Seizure free after Spencer mode topectomy. The existence of right fronto-temporal is observed as one of the dominant sub-networks as shown in Figure 4.3. The other observation is the similar pattern of wiring among different epochs of the subject. Figure 4.4 shows graph theory measurements corresponding to pre- interpost- ictal stages for different epochs across for P02. 

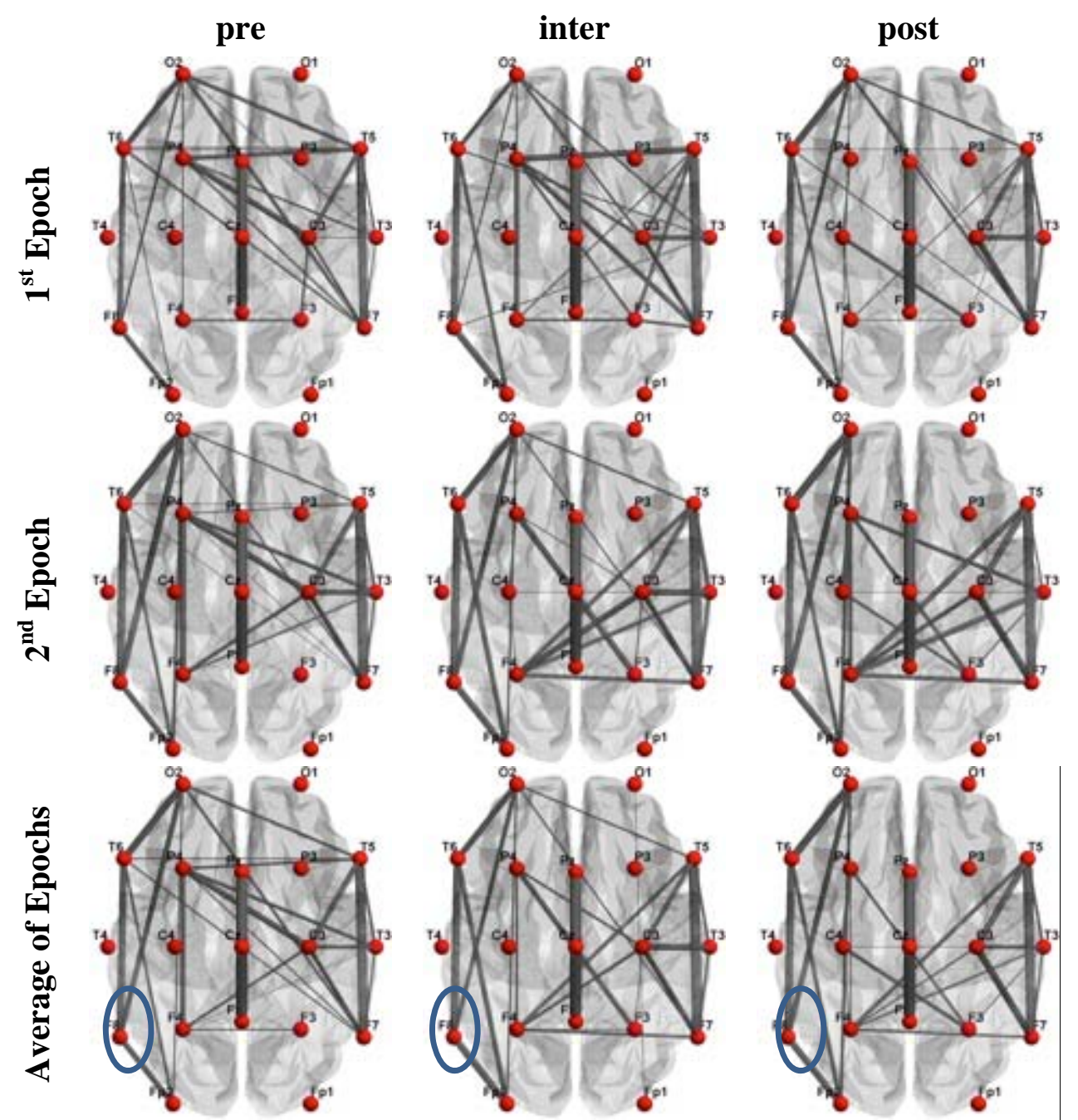

Figure 4.3: Functional connectivity networks corresponding to pre- inter- post- ictal stages for different epochs across for P02. 

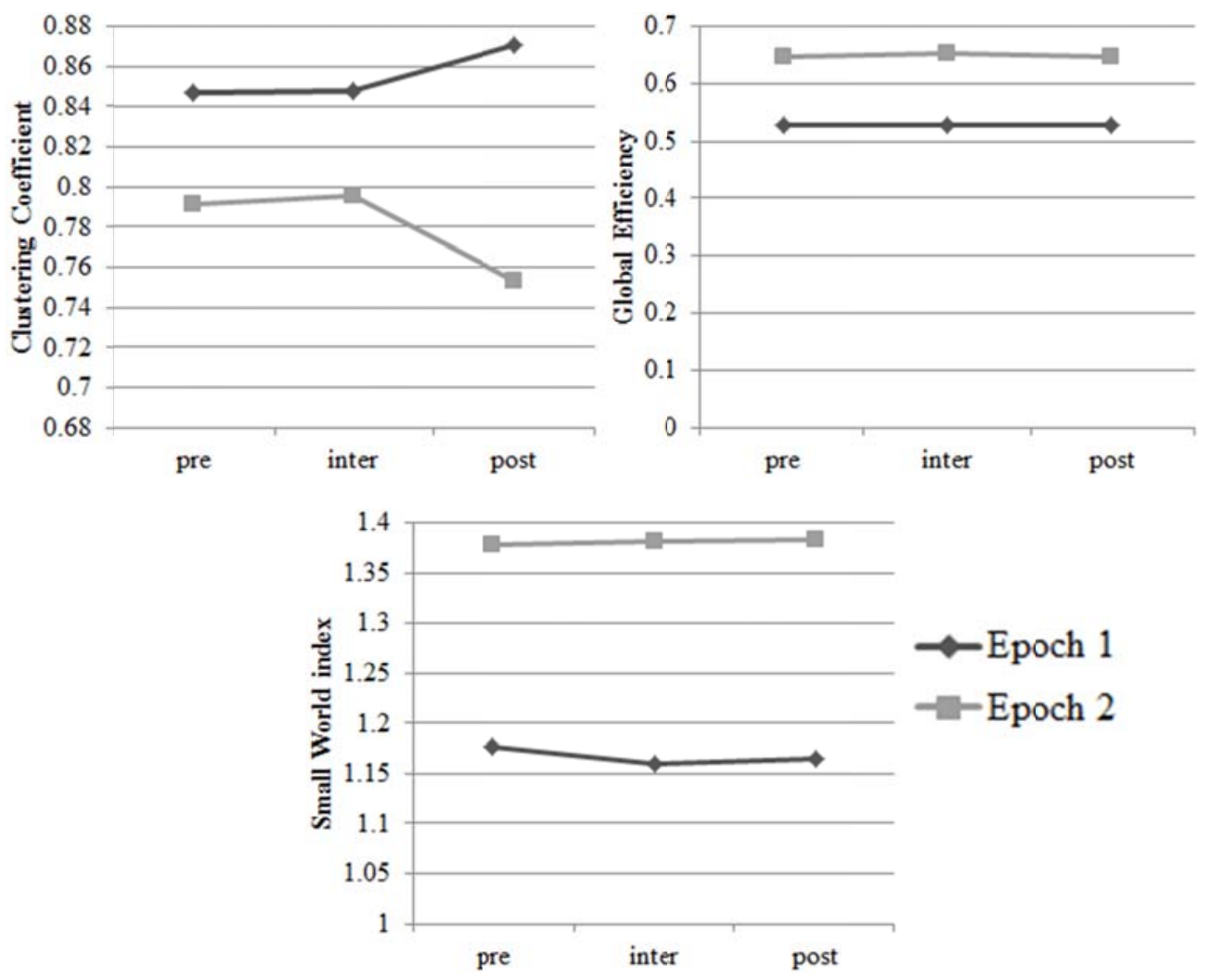

Figure 4.4: Graph Theory Measurements corresponding to pre- inter- post- ictal stages for different epochs across for P02.

P03: Having generalized seizures with focal onset (some strange feeling, vertigo for few seconds). Occasional high amplitude spiking within right fronto-central region. MRI showed typical picture of Focal Cortical Dysplasia (FCD) within right frontal lobe. Broad resection of right frontal lobe. The involvement of right fronto-central lobe in the FCNs maps in Figure 4.5 relates to the location of the mass revealed in MRI. Intra-similarity among FCN's maps for different epochs is visually observable. Figure 4.6 shows graph theory measurements corresponding to pre- inter- post- ictal stages for different epochs. 


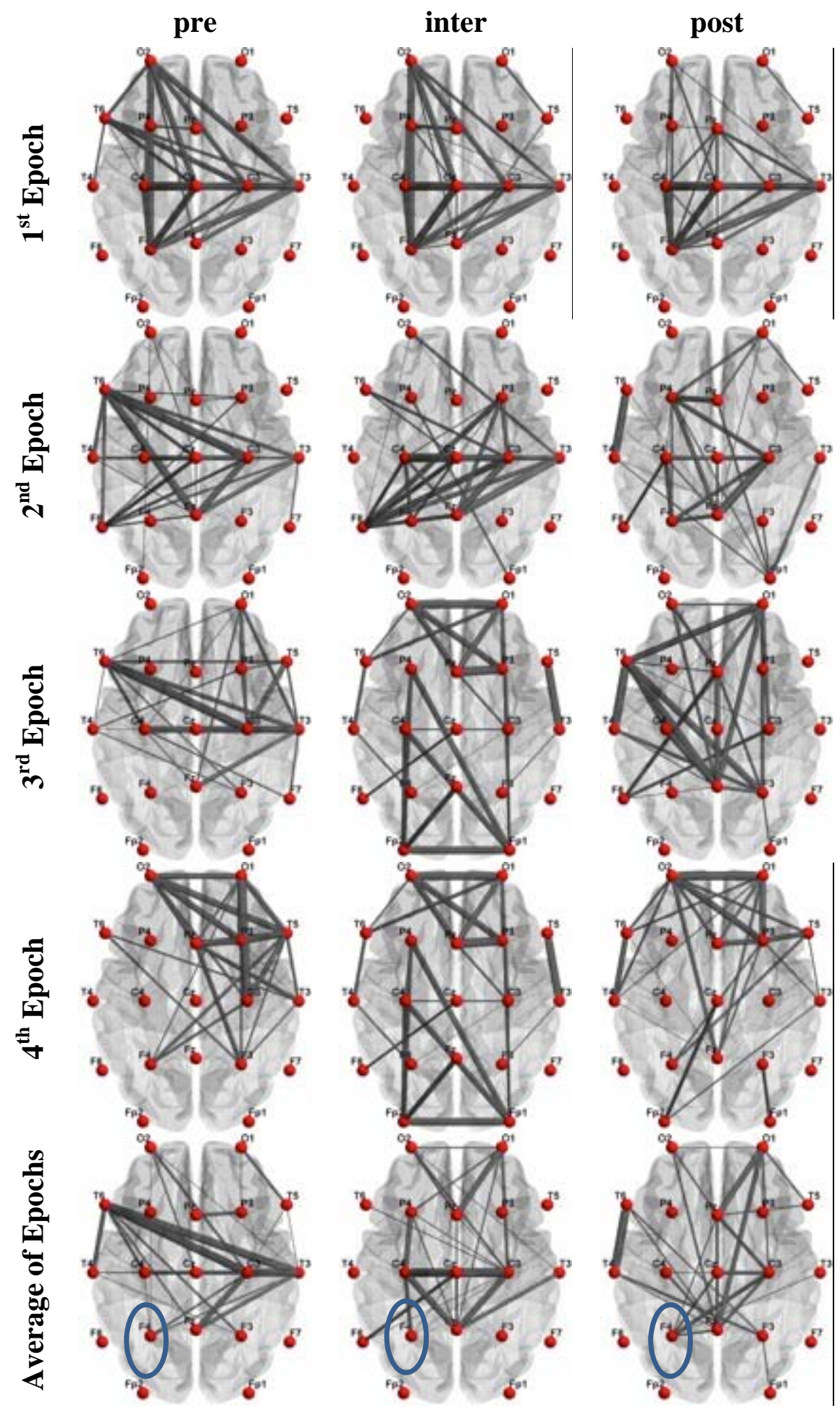

Figure 4.5: Functional connectivity networks corresponding to pre- inter- post- ictal stages for different epochs across for P03. 

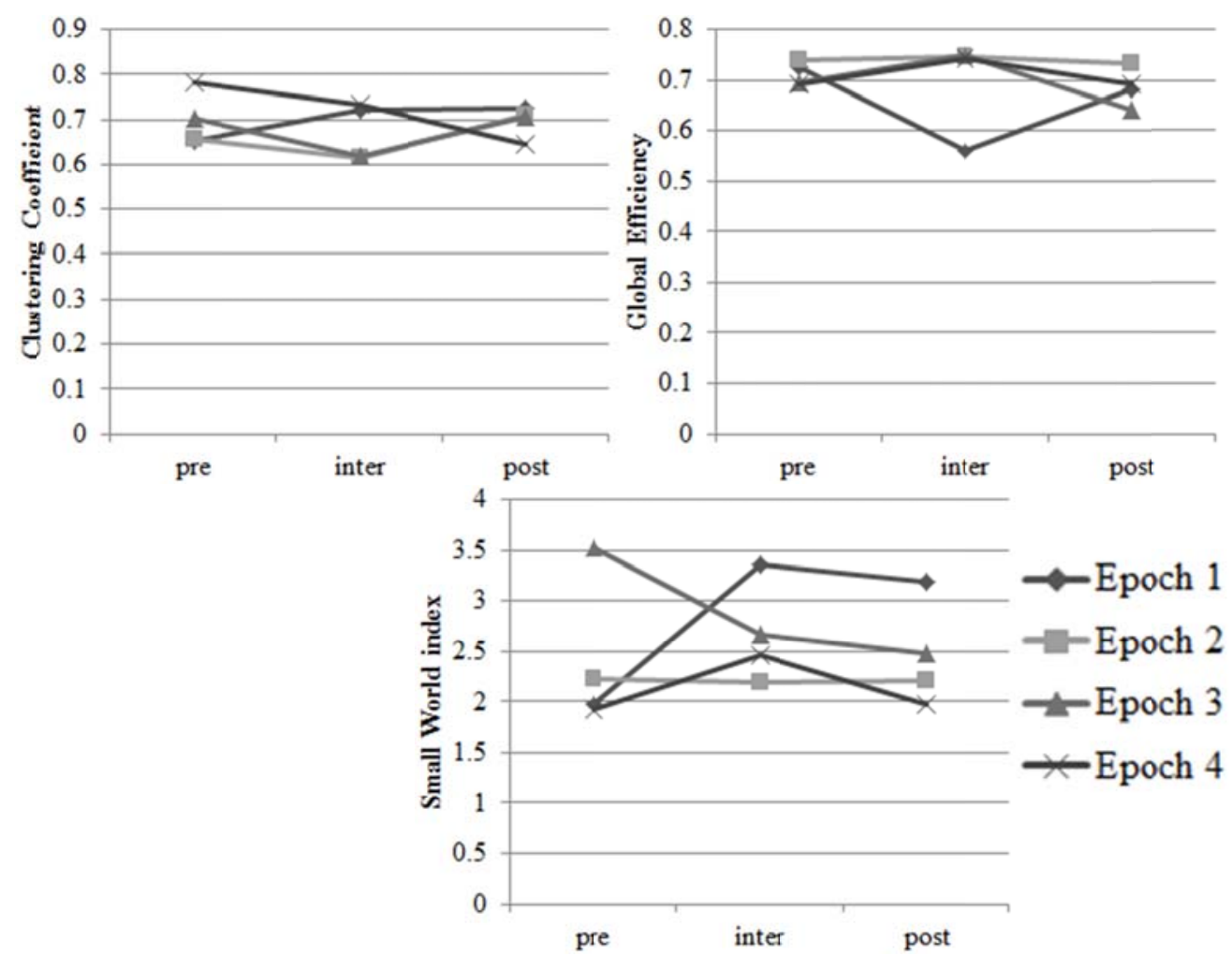

Figure 4.6: Graph Theory Measurements corresponding to pre- inter- post- ictal stages for different epochs across for P03.

P04: Severe form of temporal epilepsy. Many complex partial fits (automatisms, bizarre agitation). Mild to moderate cognitive impairment. Some repetitive synchronized gross sharp waves with regional showing in right temporal area. Seizure free after right temporal topectomy. Average FCN's maps in Figure 4.7 are not highlighting the location belonging to the existence of revealed lesion in the right mesio-temporal area which could be due to the thresholding and the high connectivity indices of right occipital networks. However, the similarities among different epochs are verified in both their maps and their corresponding graph theory measures. Figure 4.8 shows graph theory measurements corresponding to pre- inter- post- ictal stages for different epochs. 


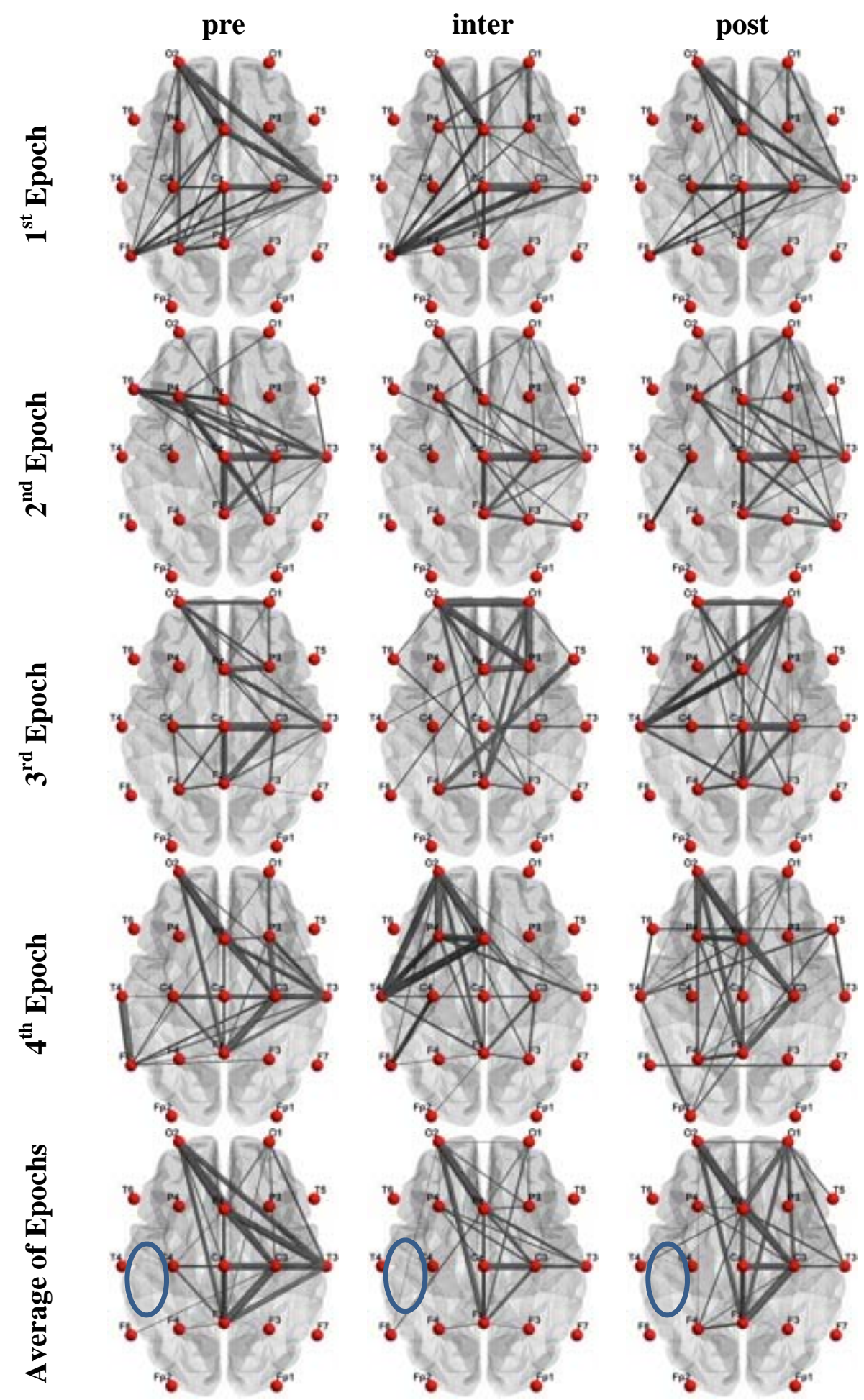

Figure 4.7: Functional connectivity networks corresponding to pre- inter- post- ictal stages for different epochs across for P04. 

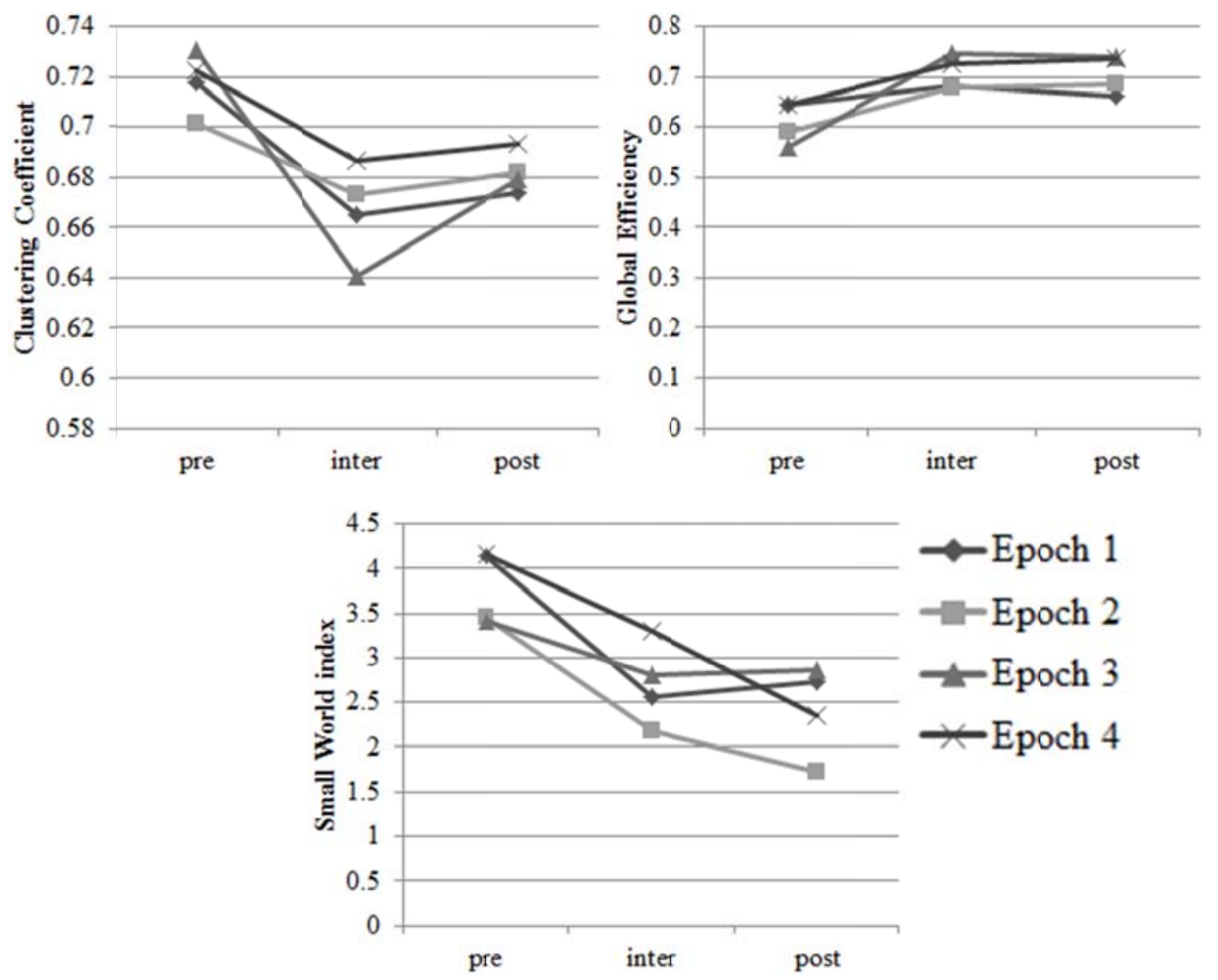

Figure 4.8: Graph Theory Measurements corresponding to pre- inter- post- ictal stages for different epochs across for P04.

P05: Mild cognitive impairment. Severe temporal seizures with mesiotemporal symptoms. Frequent ictal discharges of sharp waves and spikes generalizing from right temporal region to whole right hemisphere. MRI showed a mass within pole of the right temporal lobe as well as in mesial structures. One of the active sub-networks in Figure 4.9 matches the location of MRI-revealed mass within the right temporal lobe. Figure 4.10 shows graph theory measurements corresponding to pre- inter- post- ictal stages for different epochs. 


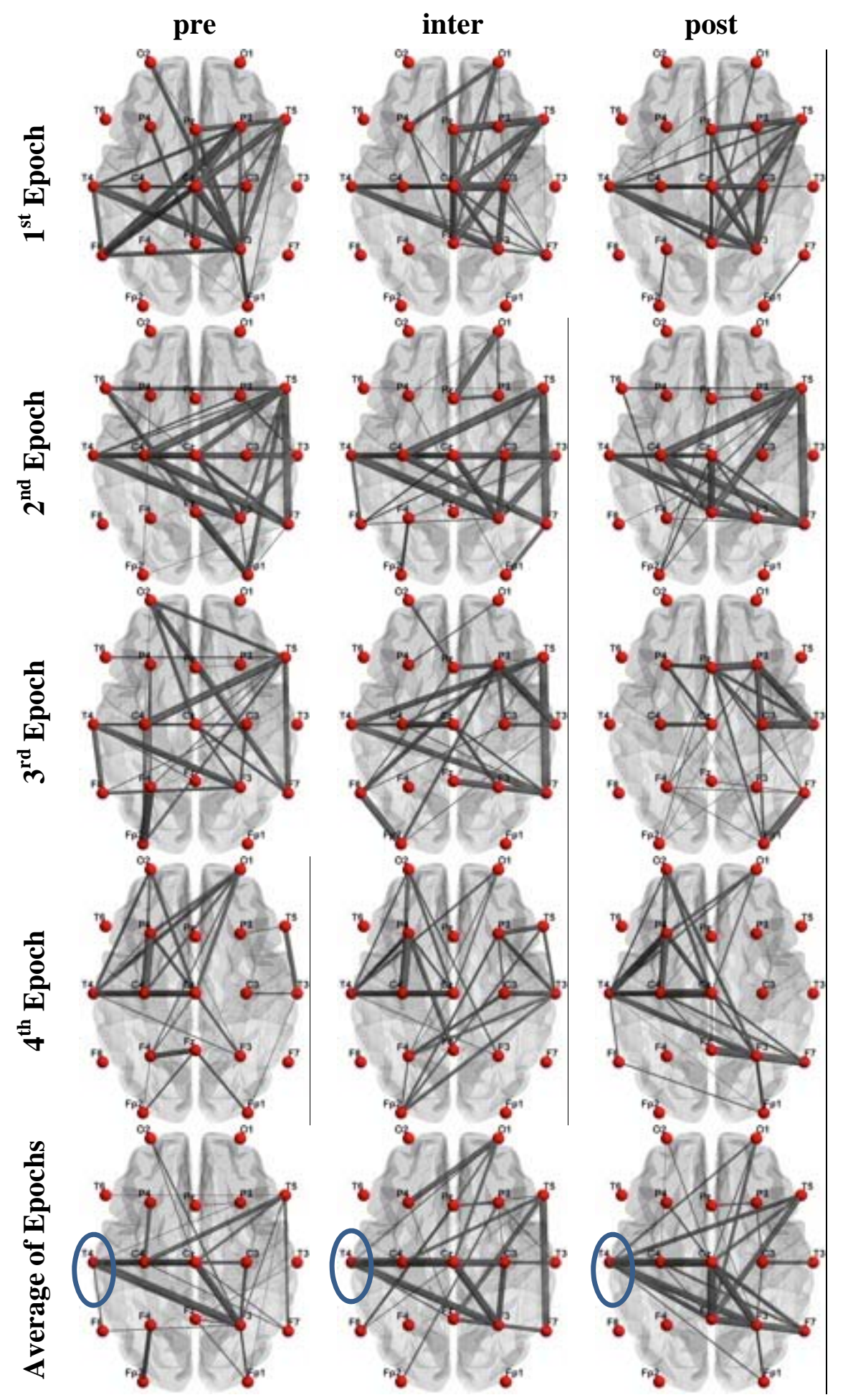

Figure 4.9: Functional connectivity networks corresponding to pre- inter- post- ictal stages for different epochs across for P05. 

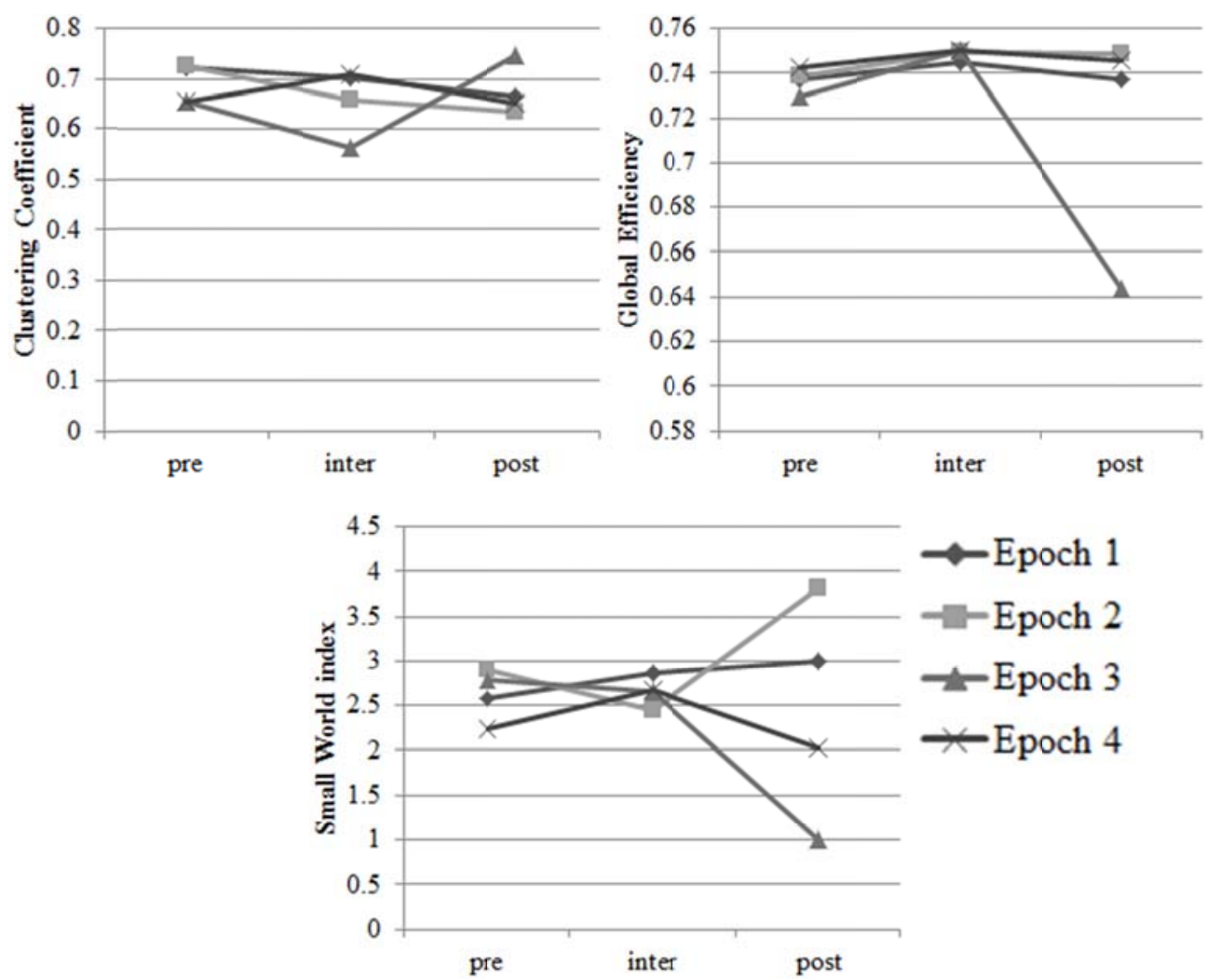

Figure 4.10: Graph Theory Measurements corresponding to pre- inter- post- ictal stages for different epochs across for P05.

P06: Atypical pseudo-absences and some occasional partial fits with mesio-temporal symptoms; unstable pattern of sharp waves and rare spiking arising from both sides. MRI showed a mass located in right temporal pole and mesial region. High correlation among the pattern of active sub-networks in FCN's maps corresponding to different epochs is visually verified. The location of the MRI-revealed mass is also recognized in the active sub-networks of the FCNs in Figure 4.11. Figure 4.12 shows graph theory measurements corresponding to pre- inter- post- ictal stages for different epochs. 


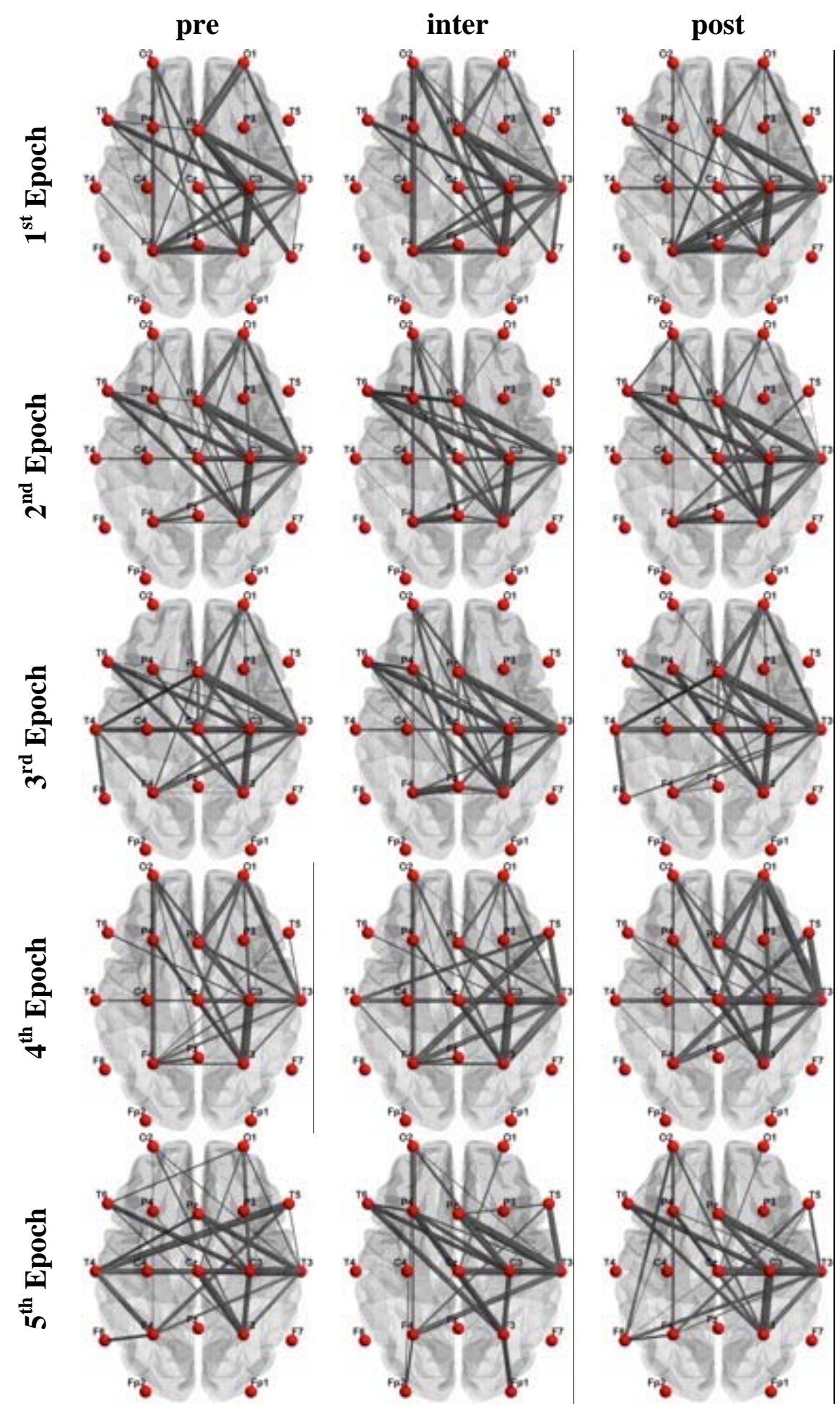

Figure 4.11 : Functional connectivity networks corresponding to pre- inter- post- ictal stages for different epochs across for P06. 


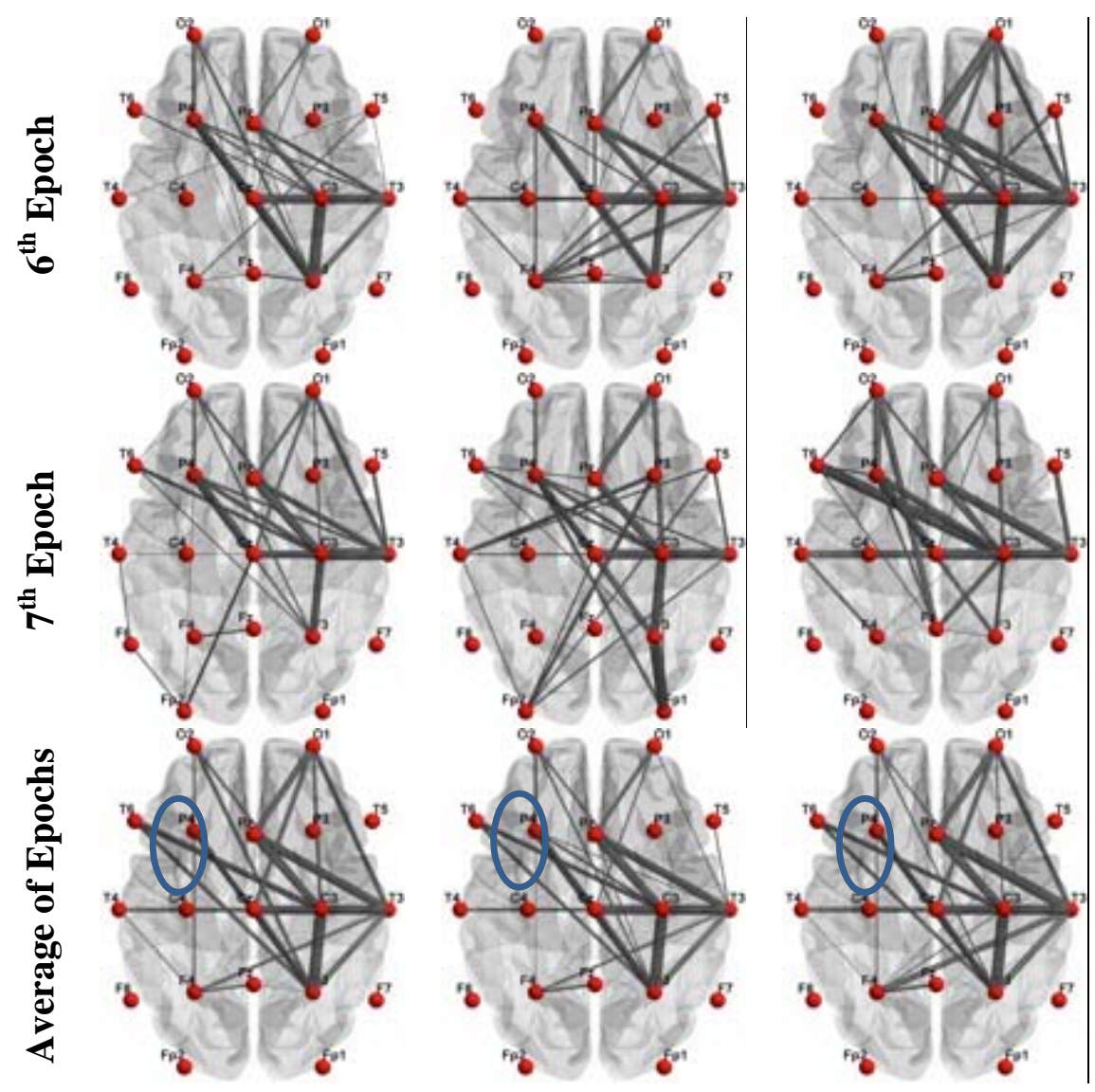

Figure 4.11 (cont.): Functional connectivity networks corresponding to pre- inter- postictal stages for different epochs across for P06.

P07: Severe form of frontal lobe seizure. Some generalized discharges with no defined point of onset. MRI showed signs of cortical dysplasia in left frontal lobe. Similar patterns of wiring scheme are observed across different epochs shown in Figure 4.13. The location of diagnosed cortical dysplasia in the left frontal lobe is exhibited as one of the active sub-networks in FCN's maps. Figure 4.14 shows graph theory measurements corresponding to pre- inter- post- ictal stages for different epochs. 

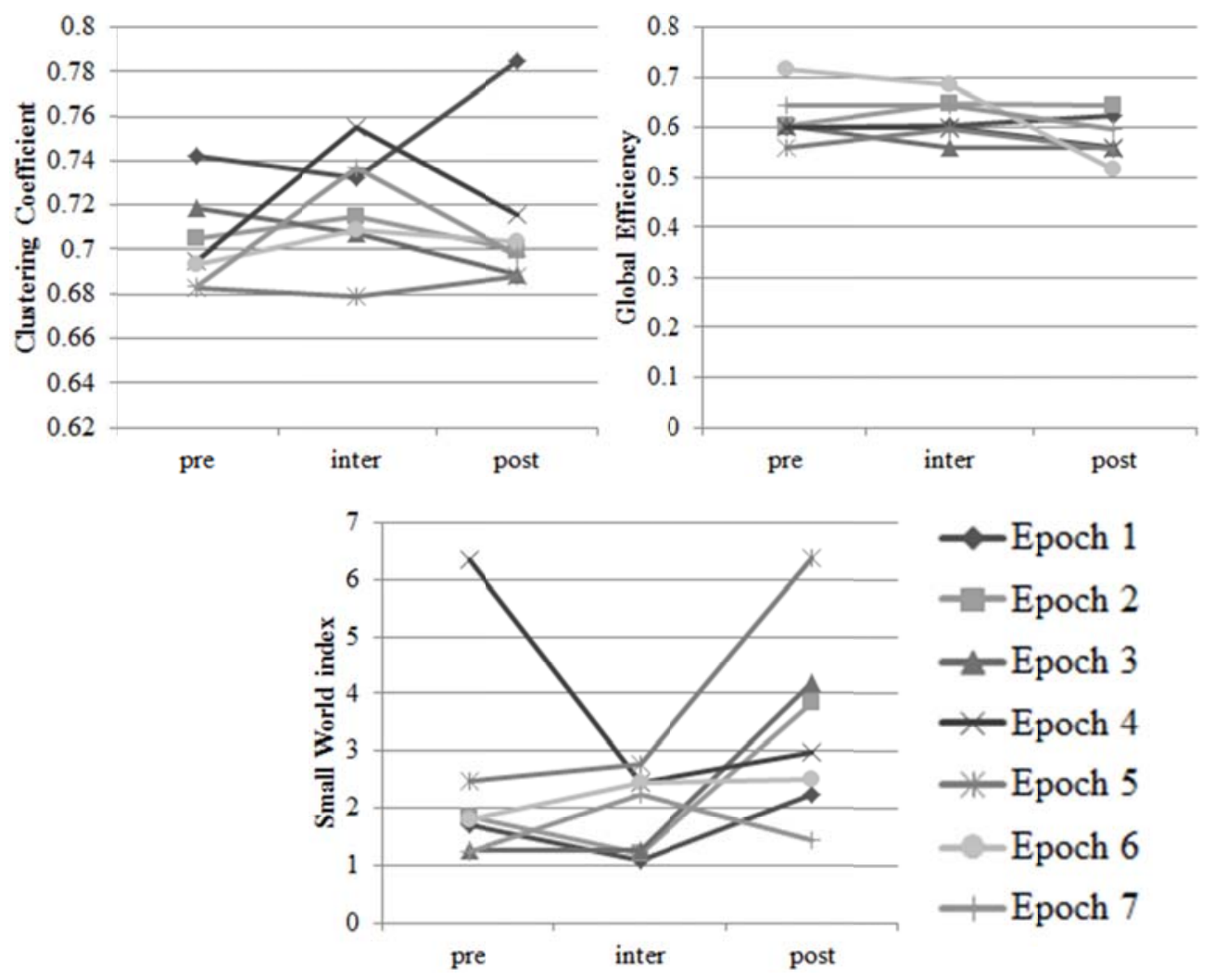

Figure 4.12: Graph Theory Measurements corresponding to pre- inter- post- ictal stages for different epochs across for P06.

P08: Normal EEG. MRI showed a mass within left temporal lobe. One of the subnetworks highlighted in the left temporal lobe is related to the mass found in MRI of the subject in the same area. Visual inspection of FCN's maps corresponding to different epochs are showing similar wiring schemes shown in Figure 4.15. Figure 4.16 shows graph theory measurements corresponding to pre- inter- post- ictal stages for different epochs. 


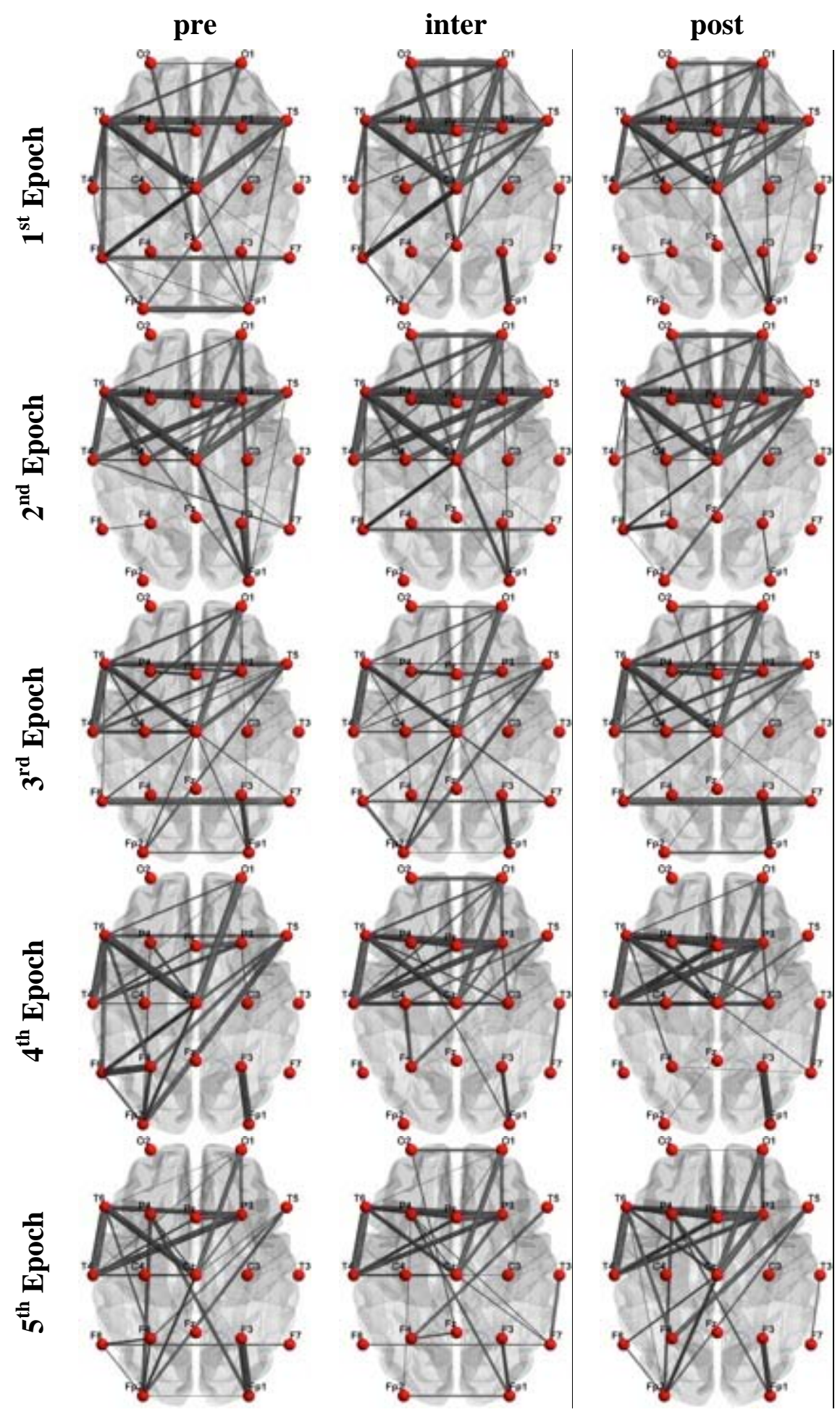

Figure 4.13: Functional connectivity networks corresponding to pre- inter- post- ictal stages for different epochs across for P07. 

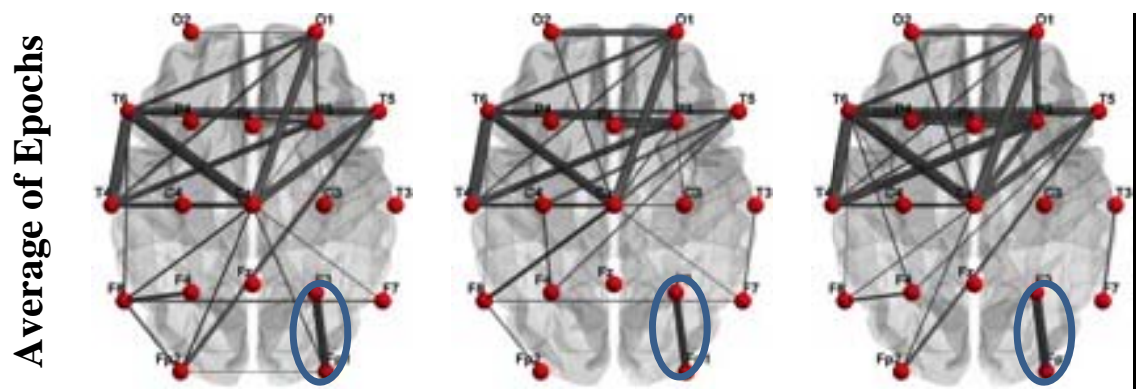

Figure 4.13 (cont.): Functional connectivity networks corresponding to pre- inter- postictal stages for different epochs across for P07.
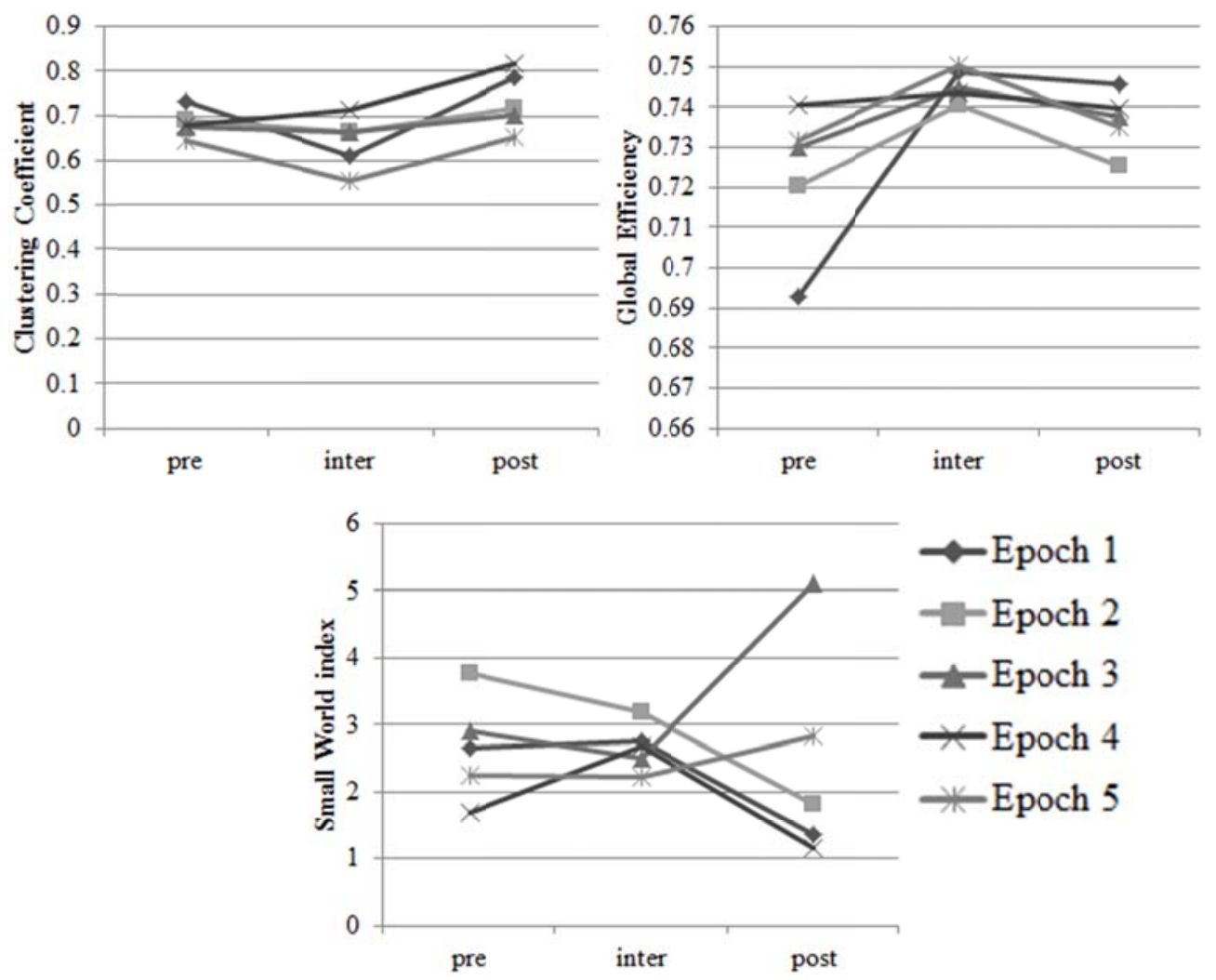

Figure 4.14: Graph Theory Measurements corresponding to pre- inter- post- ictal stages for different epochs across for P07.

P09: EEG showed long traces of mid and posterior right temporal discharges, MRI revealed a mass within mesio-temporal structures of right temporal lobe. The FCN's maps in Figure 4.17 show similar evolutionary pattern of interictal events across different 
epochs for the subject. The location of the MRI mass within the mid and posterior temporal lobe is also recognized in the maps. Figure 4.18 shows graph theory measurements corresponding to pre- inter- post- ictal stages for different epochs.

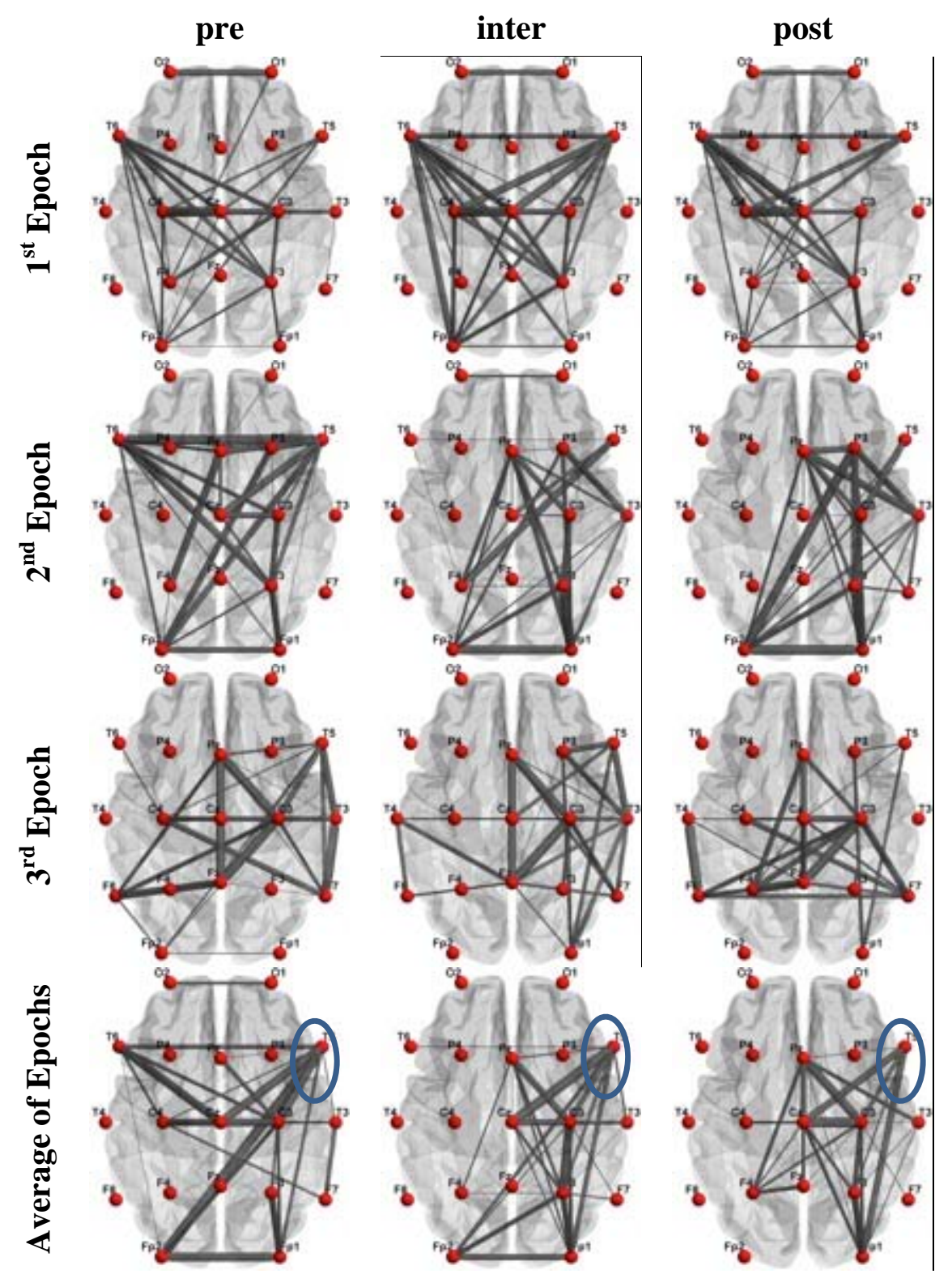

Figure 4.15: Functional connectivity networks corresponding to pre- inter- post- ictal stages for different epochs across for P08. 

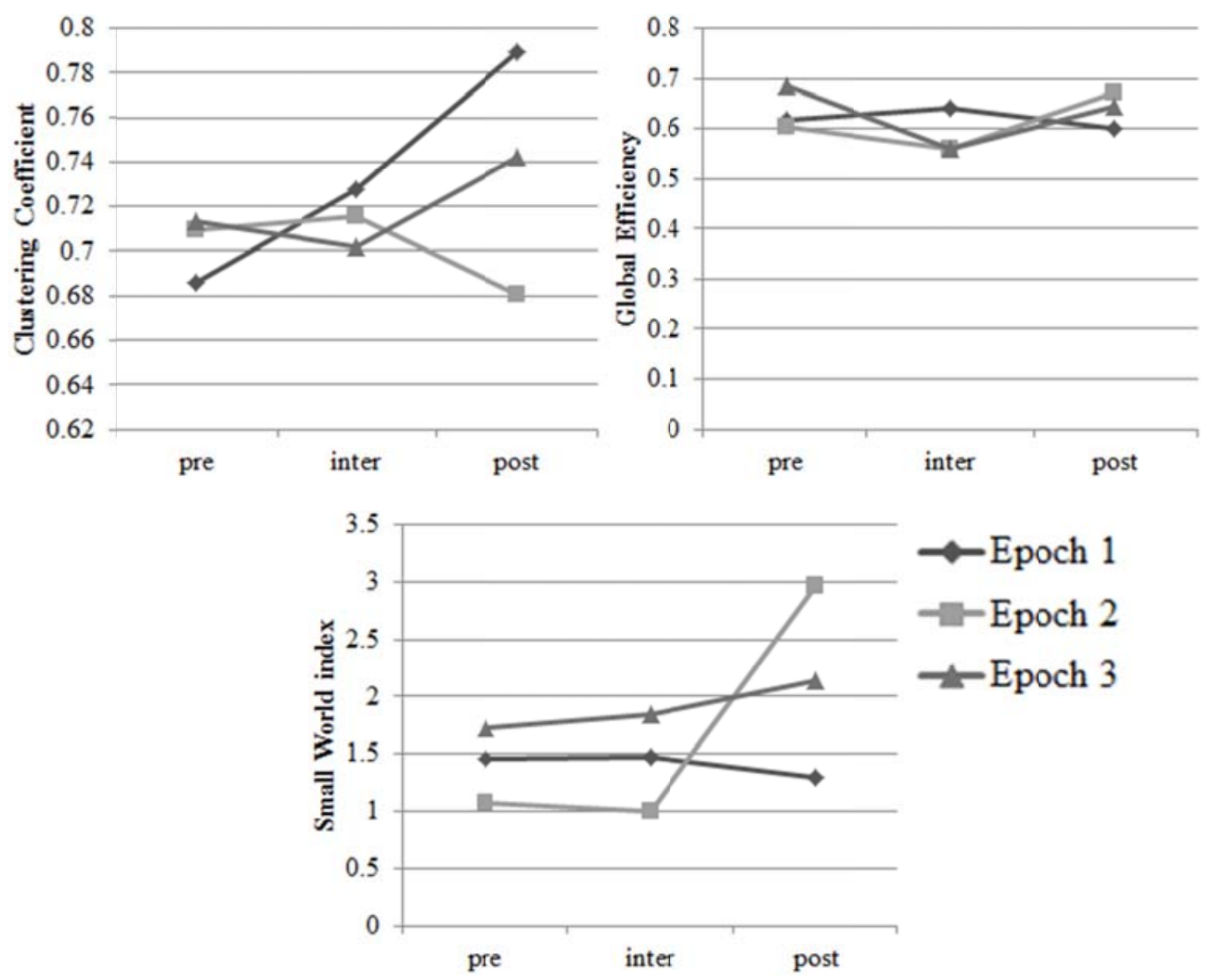

Figure 4.16: Graph Theory Measurements corresponding to pre- inter- post- ictal stages for different epochs across for P08.

P10: EEG showed intermittent traces of spiking and slow wave activity within right temporal region. MRI showed cystic tumor within mesiotemporal structures of right temporal lobe. A Similar network wiring scheme is observed among different epochs in Figure 4.19. However, the location of the MRI-revealed mass is partially a fit with the active sub-network, which could be due to the large impact of the occipital lobe subnetwork. Figure 4.20 shows graph theory measurements corresponding to pre- inter- postictal stages for different epochs. 


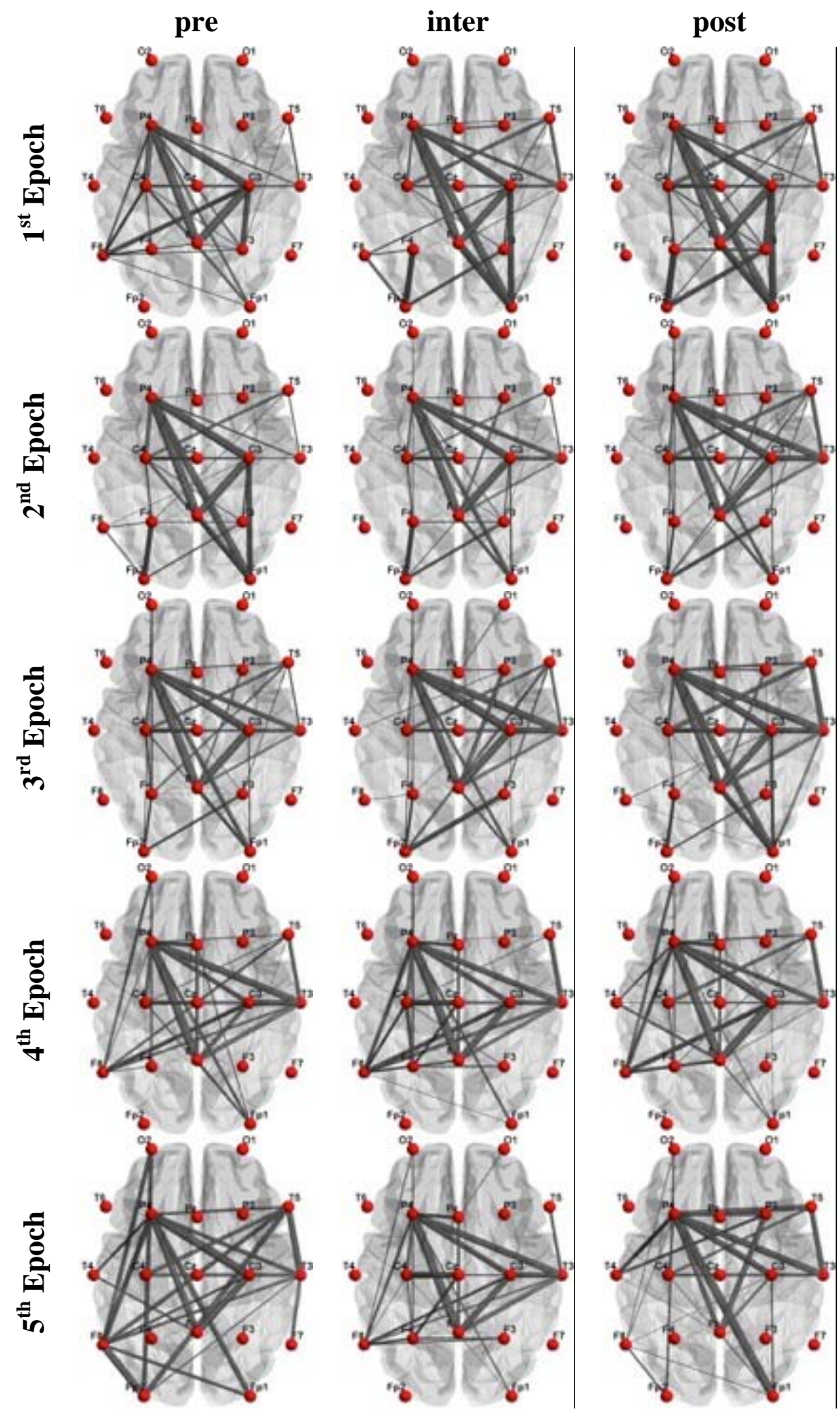

Figure 4.17: Functional connectivity networks corresponding to pre- inter- post- ictal stages for different epochs across for P09. 

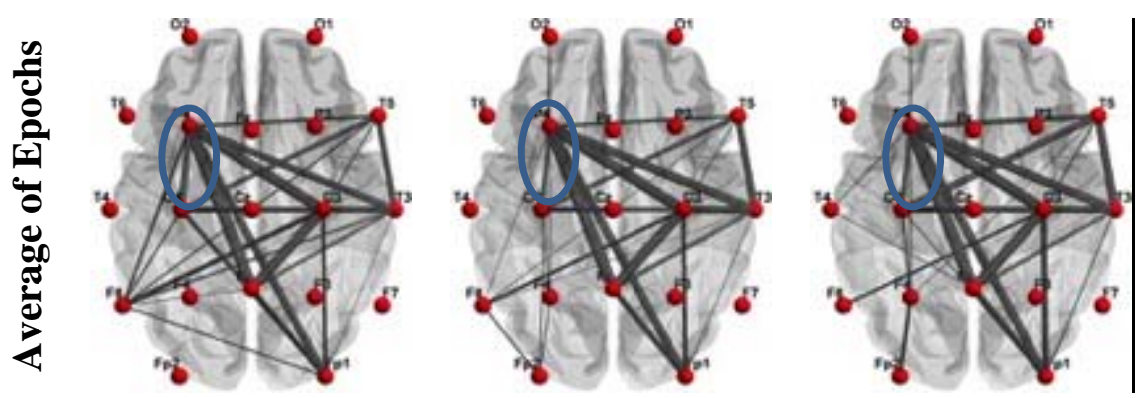

Figure 4.17 (cont.): Functional connectivity networks corresponding to pre- inter- postictal stages for different epochs across for P09.
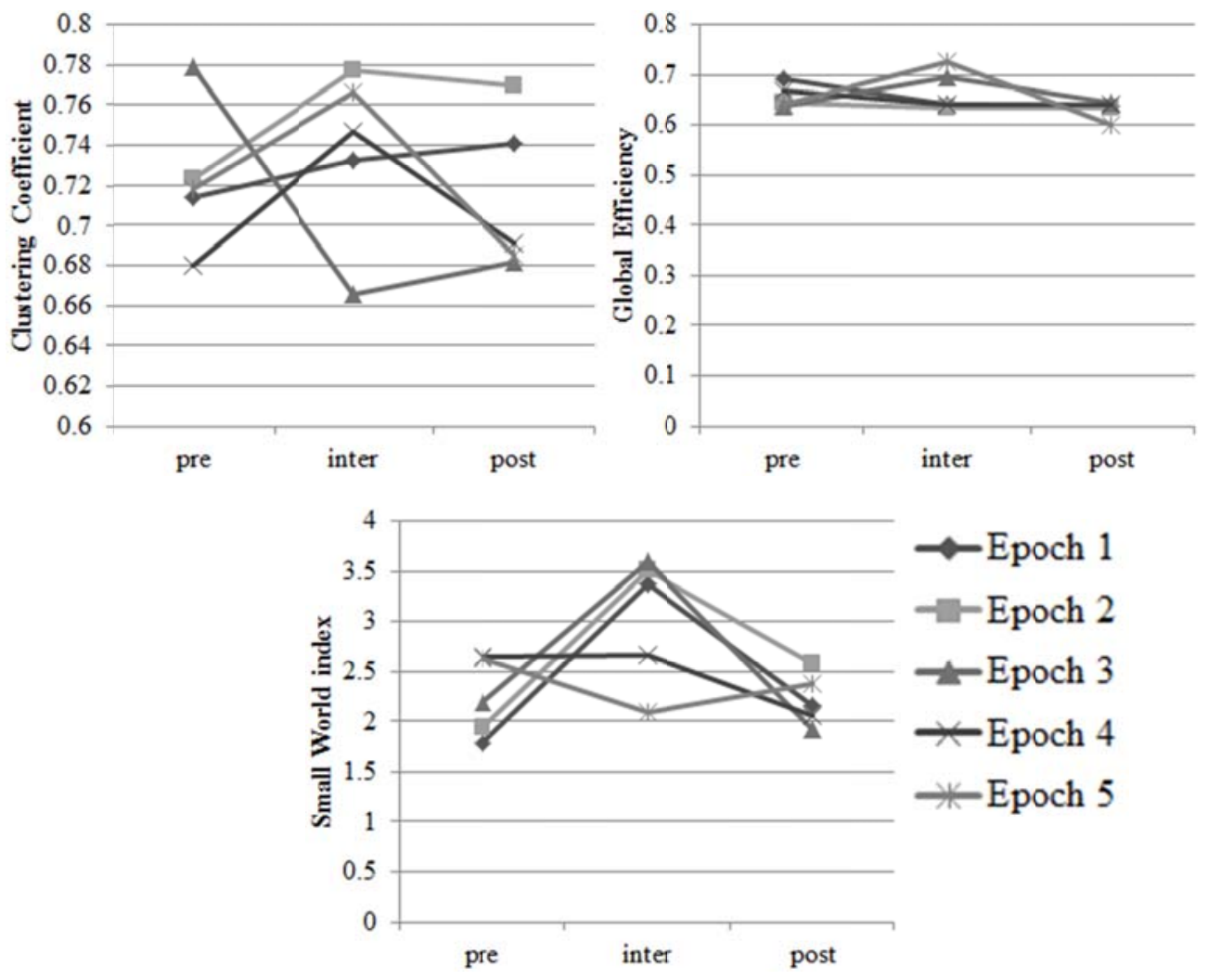

Figure 4.18: Graph Theory Measurements corresponding to pre- inter- post- ictal stages for different epochs across for P09. 


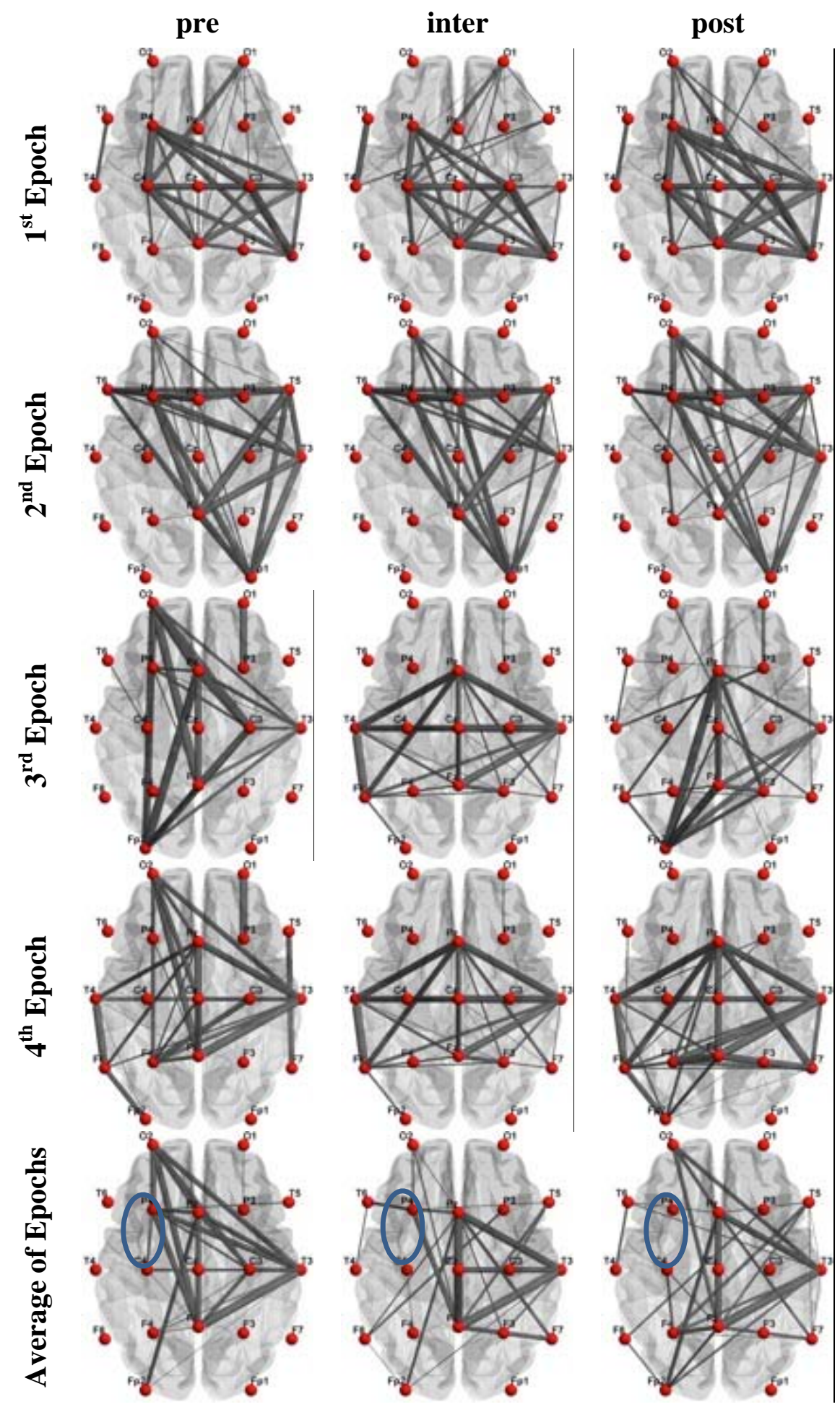

Figure 4.19: Functional connectivity networks corresponding to pre- inter- post- ictal stages for different epochs across for P10. 

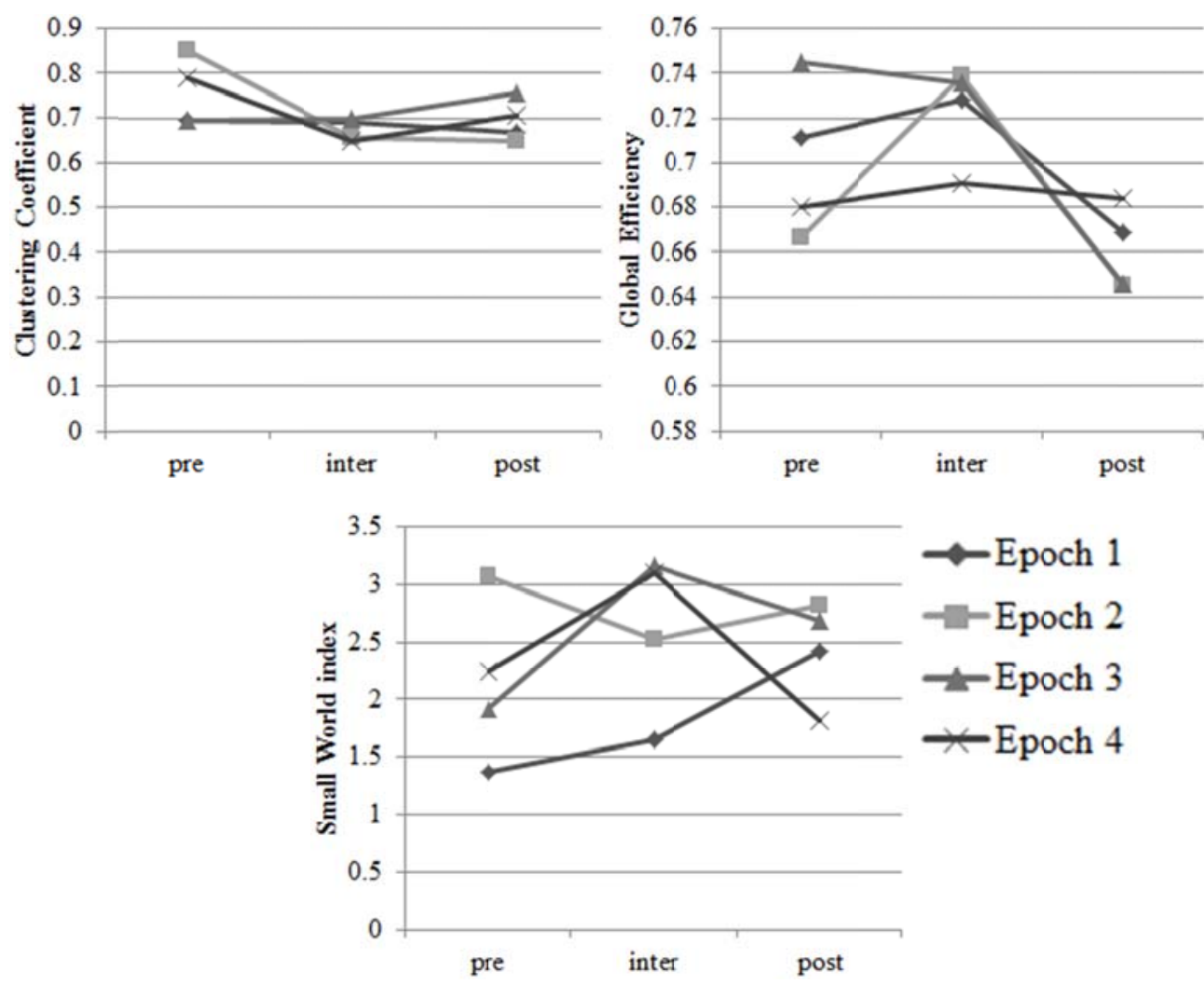

Figure 4.20: Graph Theory Measurements corresponding to pre- inter- post- ictal stages for different epochs across for P10.

P11: Severe generalized epilepsy, EEG revealed very discrete discharges within left partieto-central sharp waves, MRI showed a pathologic mass within left parietal lobe, seizure free only for two months after lesionectomy. As for the wiring distribution, similar patterns were found among different epochs as shown in Figure 4.21. Left parieto-central lobe mass revealed in MRI of the subject is in correspondence with the FCN's maps. Figure 4.22 shows graph theory measurements corresponding to pre- interpost- ictal stages for different epochs. 


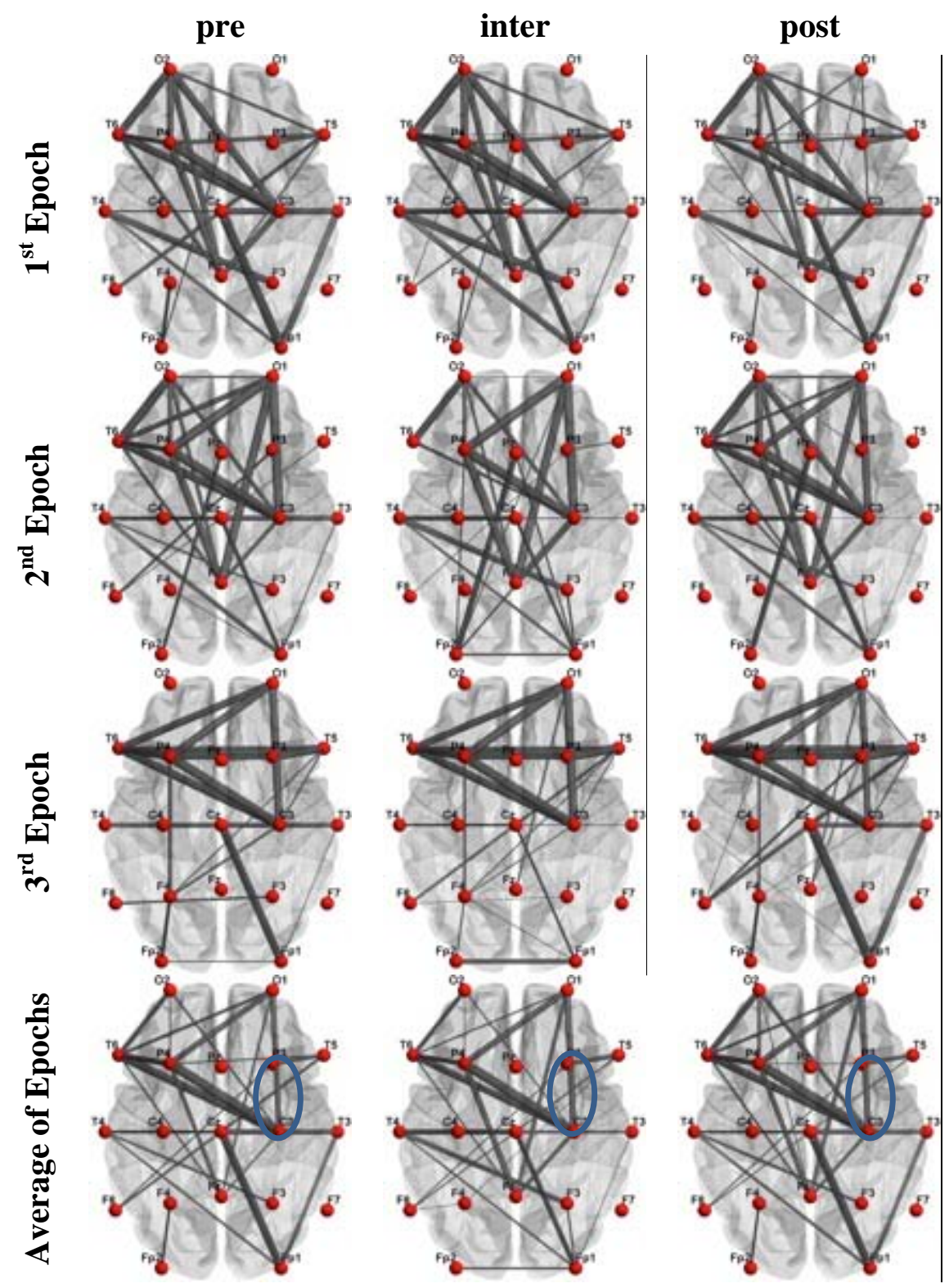

Figure 4.21: Functional connectivity networks corresponding to pre- inter- post- ictal stages for different epochs across for P11. 

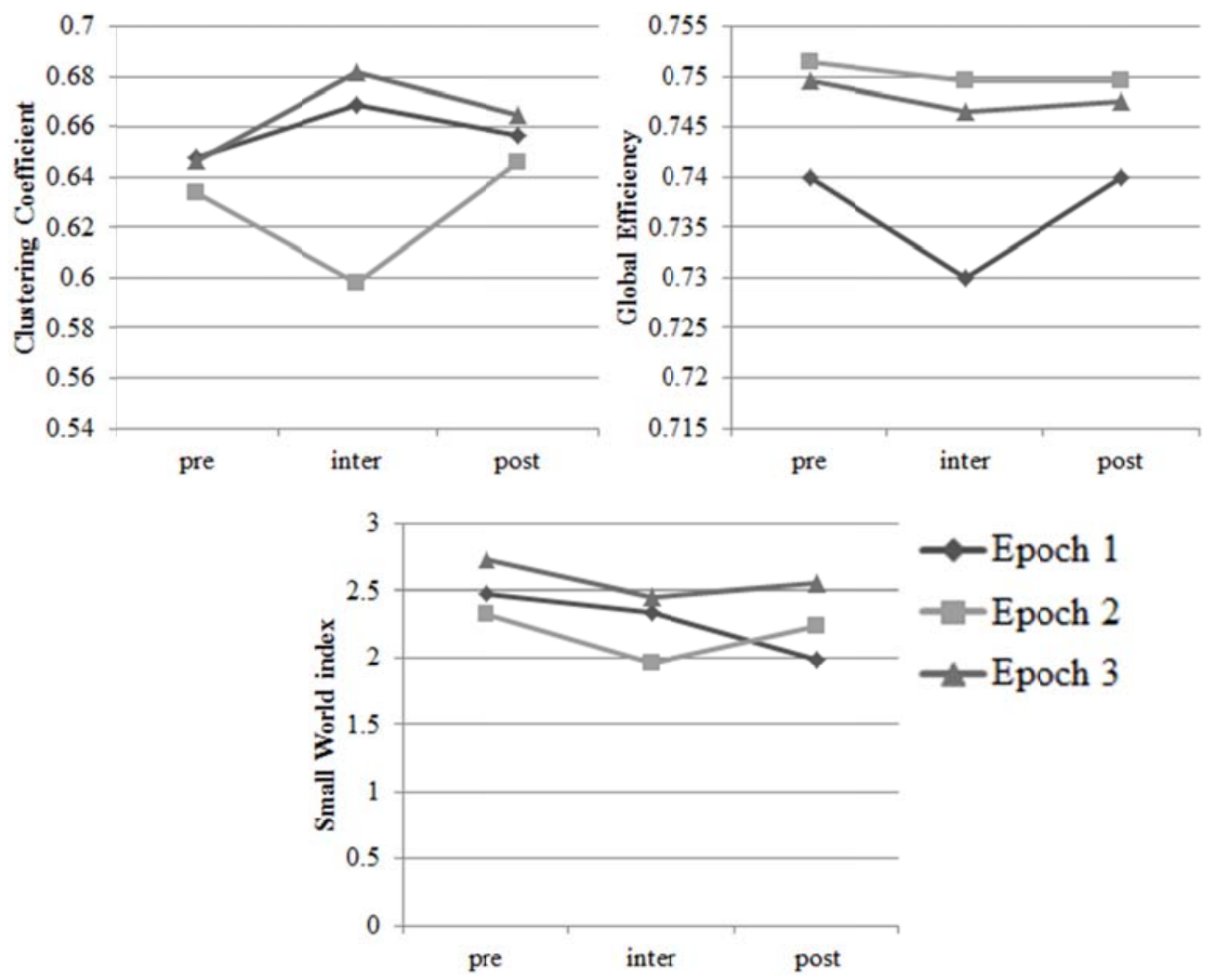

Figure 4.22: Graph Theory Measurements corresponding to pre- inter- post- ictal stages for different epochs across for P11.

P12: EEG showed very mild right side low-amplitude discharges, MRI showed a tumor residing in right cingular gyrus region. Observed FCN's maps shown in Figure 4.23 are recognizing the MRI-revealed mass in the right hemisphere of the subject. Figure 4.24 shows graph theory measurements corresponding to pre- inter- post- ictal stages for different epochs.

P13: EEG showed traces of left-temporal-region slow wave activity with seldom sharp waves. MRI revealed MTLE picture. Distributions of connections in FCN's maps shown in Figure 4.25 are sharing a very similar pattern in which side and focus of the epilepsy is 
highlighted. Figure 4.26 shows graph theory measurements corresponding to pre- interpost- ictal stages for different epochs.

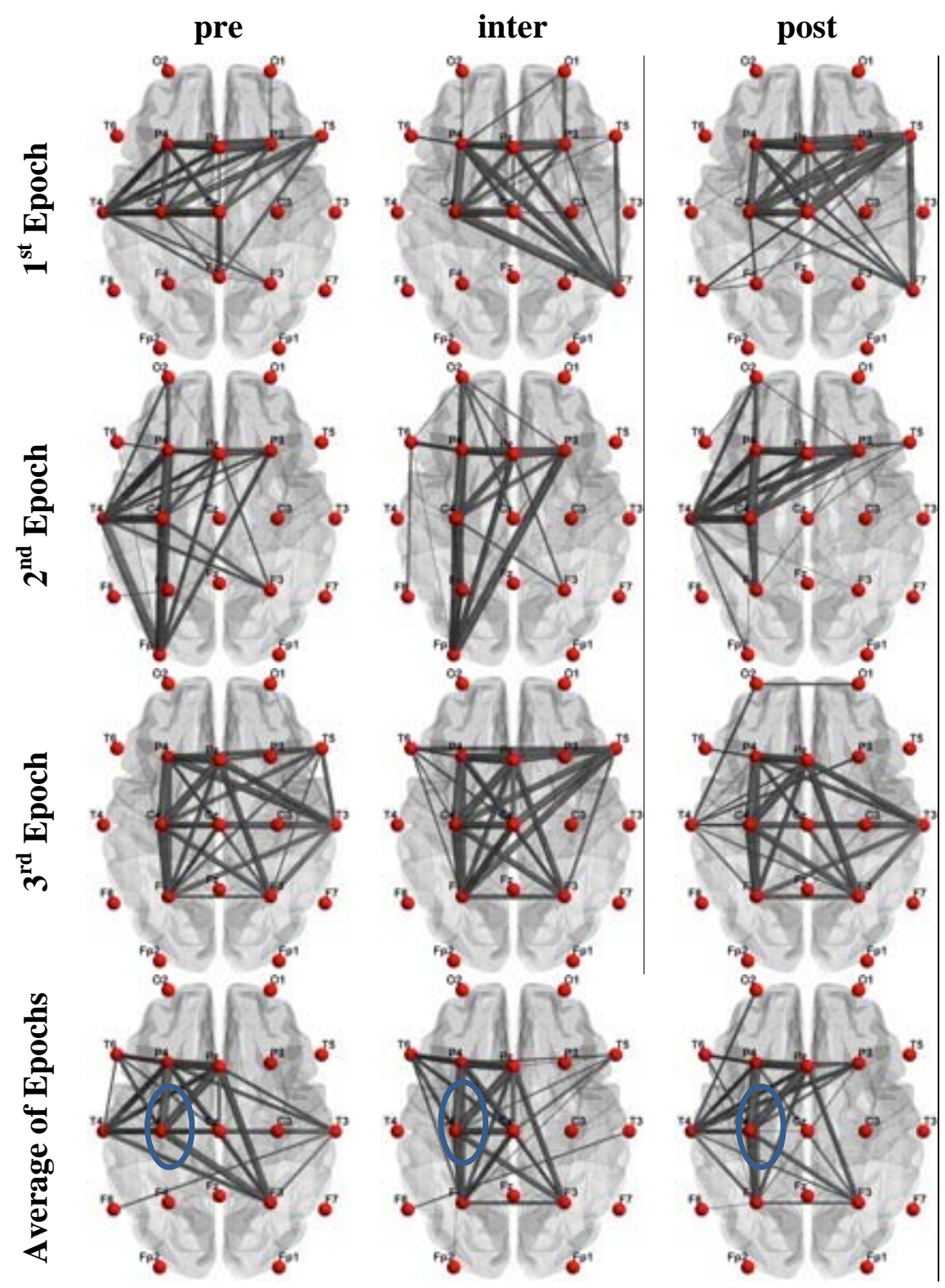

Figure 4.23: Functional connectivity networks corresponding to pre- inter- post- ictal stages for different epochs across for P12. 

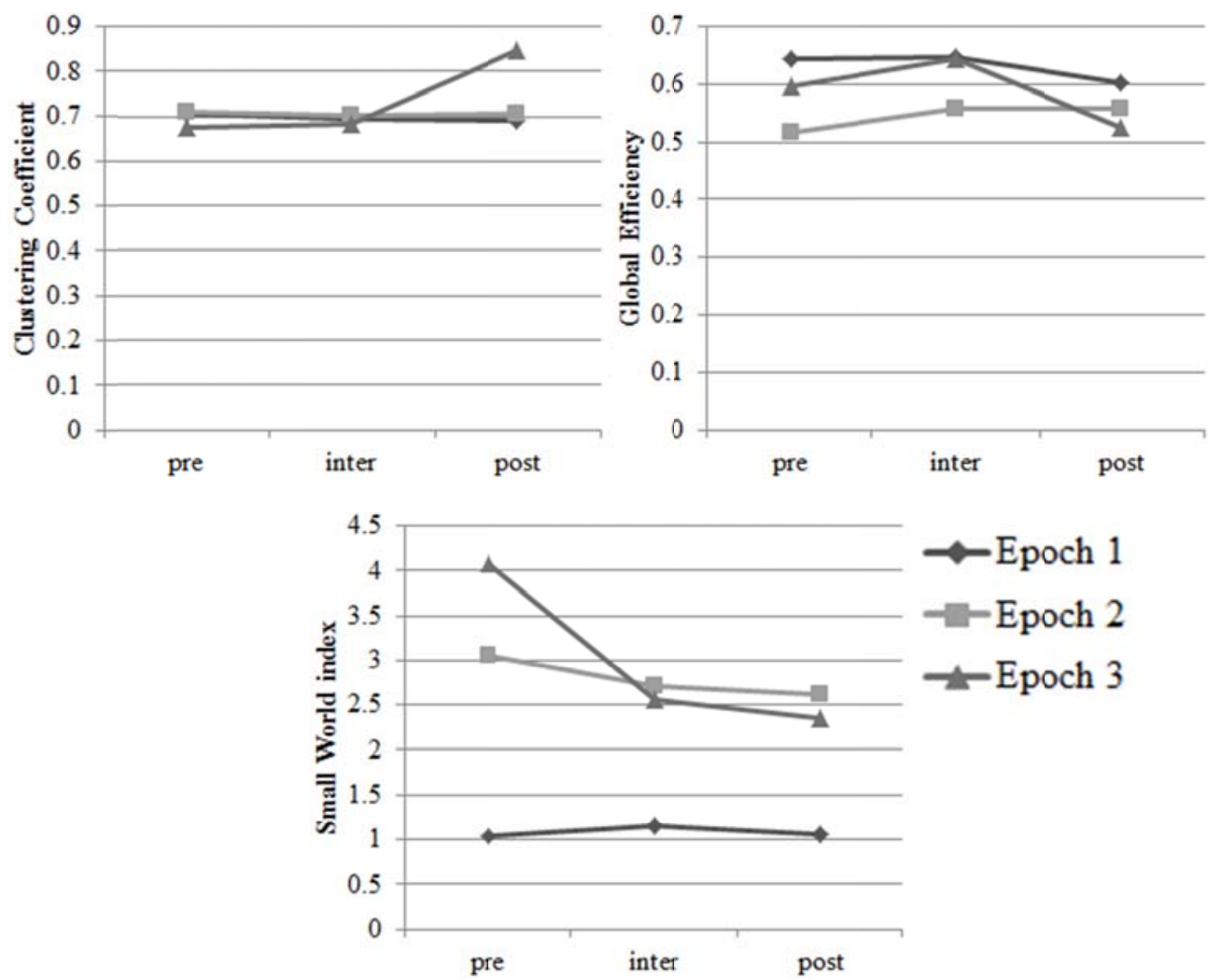

Figure 4.24: Graph Theory Measurements corresponding to pre- inter- post- ictal stages for different epochs across for P12.

P14: EEG showed traces of low-amplitude spiking within left temporal region. MRI showed MTLE within left temporal region. Wiring schemes across FCN's maps shown in Figure 4.27 of different epochs are very similar to each other and they fully match the diagnosed location and side of the focal epilepsy in the left temporal region. Figure 4.28 shows graph theory measurements corresponding to pre- inter- post- ictal stages for different epochs. 


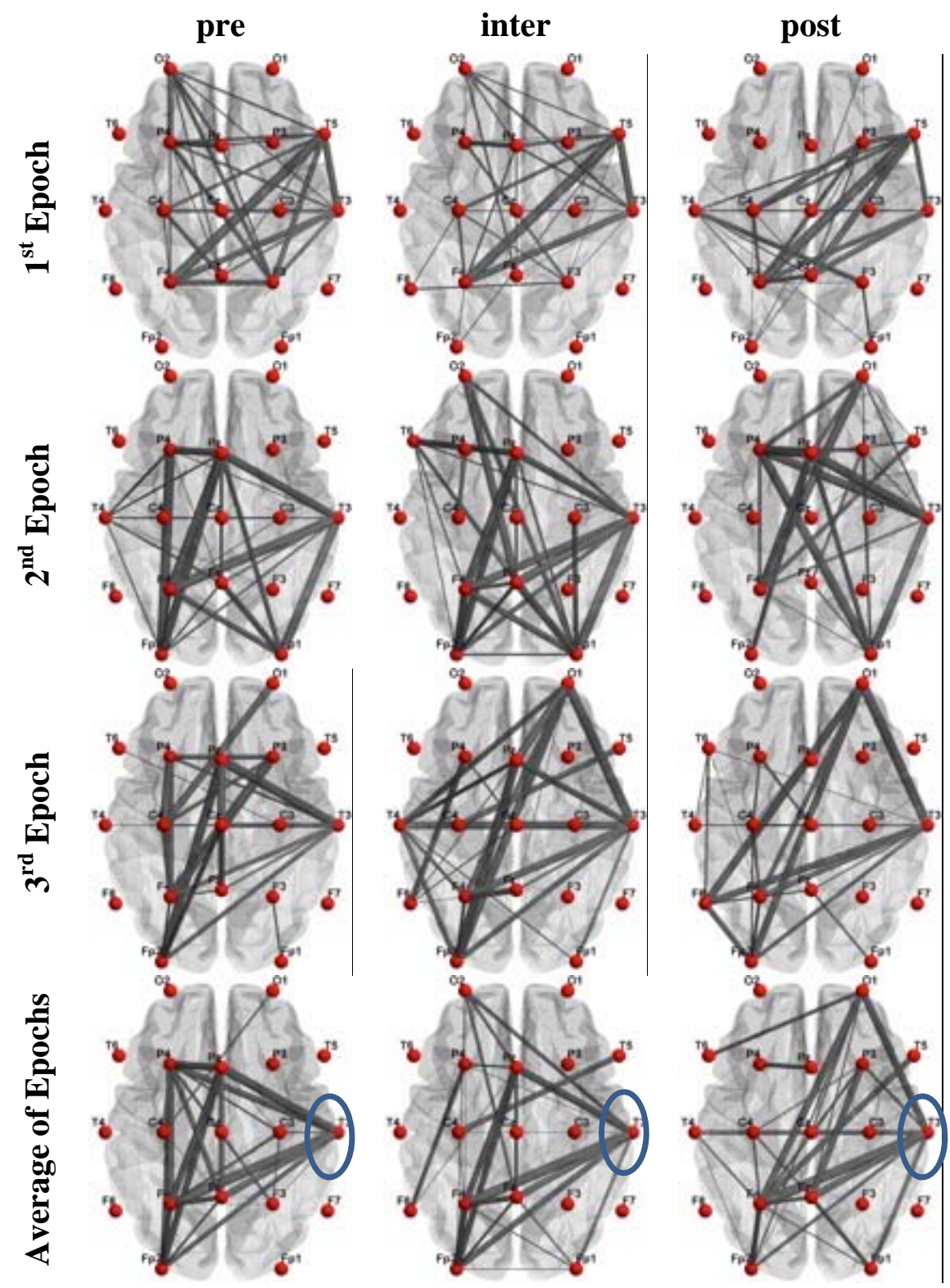

Figure 4.25: Functional connectivity networks corresponding to pre- inter- post- ictal stages for different epochs across for P13.

P15: EEG showed generalized discharges with predominant spiking in right frontal lobe. MRI showed FCD within structure of right frontal lobe. FCN's maps shown in Figure 4.29 corresponding to different epochs are showing the right frontal lobe as one of the active sub-networks, which relates to the diagnosed side and focus of epilepsy. Figure 
4.30 shows graph theory measurements corresponding to pre- inter- post- ictal stages for different epochs.
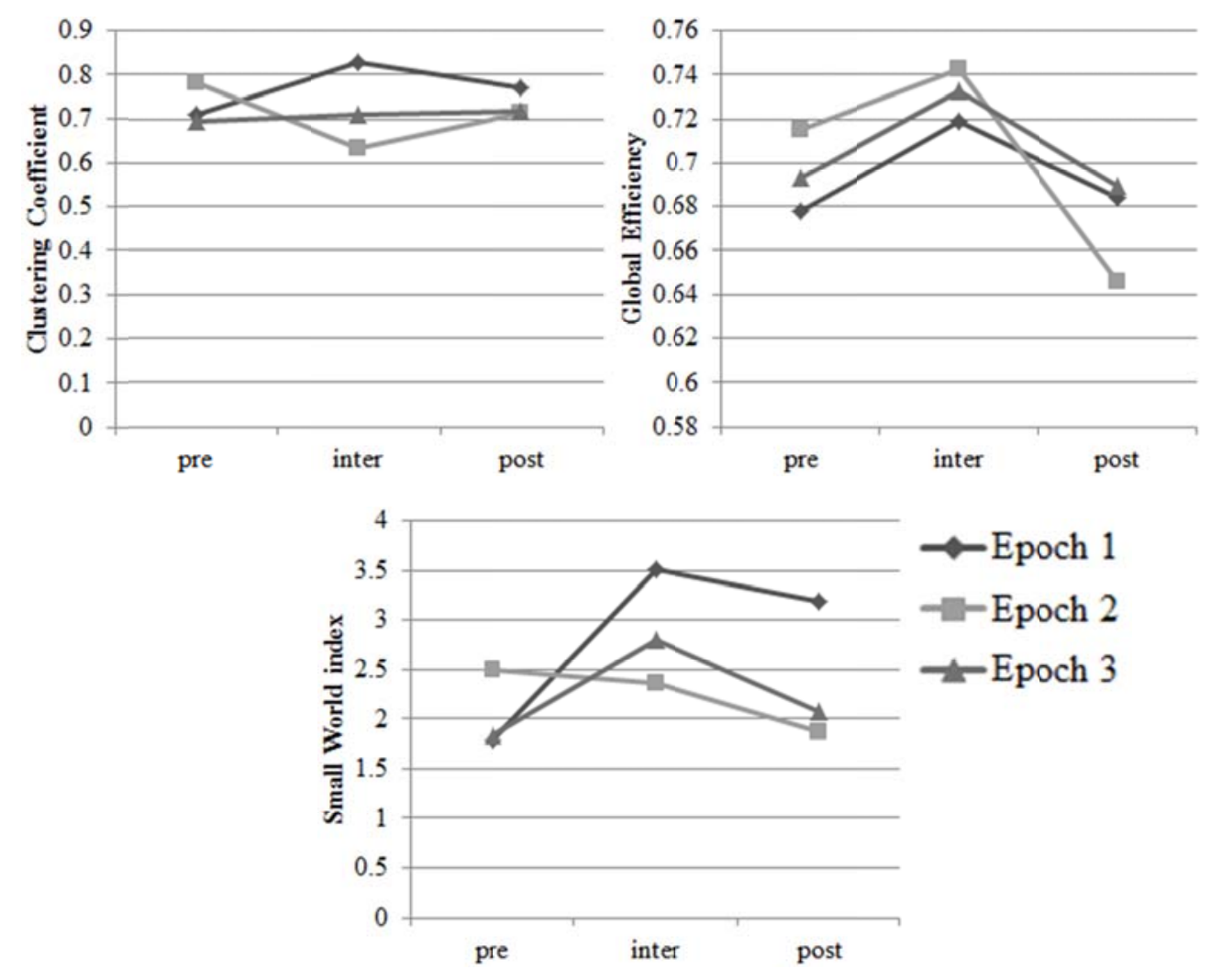

Figure 4.26: Graph Theory Measurements corresponding to pre- inter- post- ictal stages for different epochs across P13.

\subsubsection{Group Analysis}

Different stages (pre, inter and post) of the evolution of interictal events were tested (paired t-test) for the difference in three group-wised network measures (Clustering Coefficient, Graph Efficiency and Small World index). Figure 4.31 shows the variation of mean and standard deviation of three network measures.

Table 4.2 summarizes the two-tailed paired t-test results of the means of each network measure across group for different stages of the evolution of interictal recordings. Significant findings are bold highlighted. 


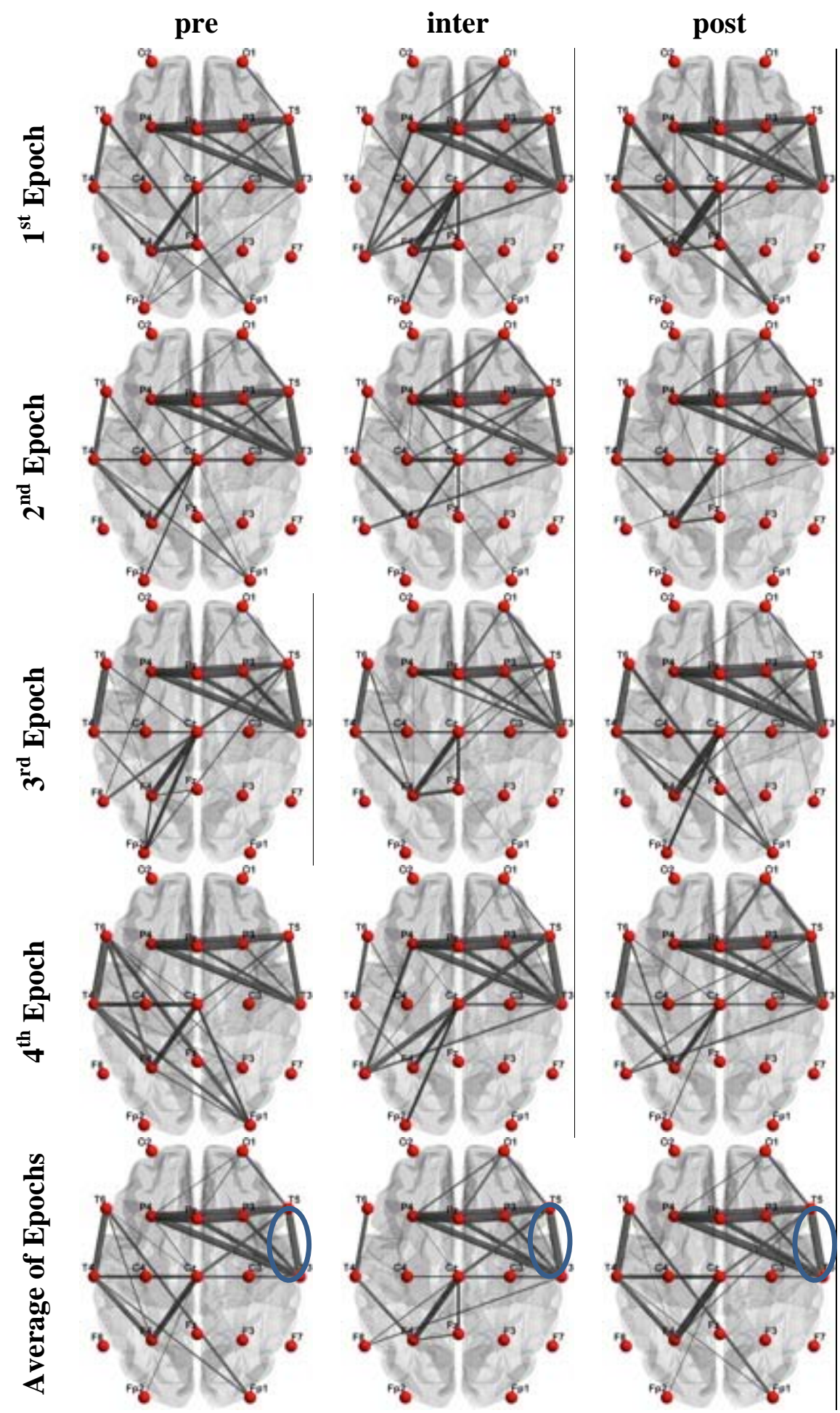

Figure 4.27: Functional connectivity networks corresponding to pre- inter- post- ictal stages for different epochs across for P14. 

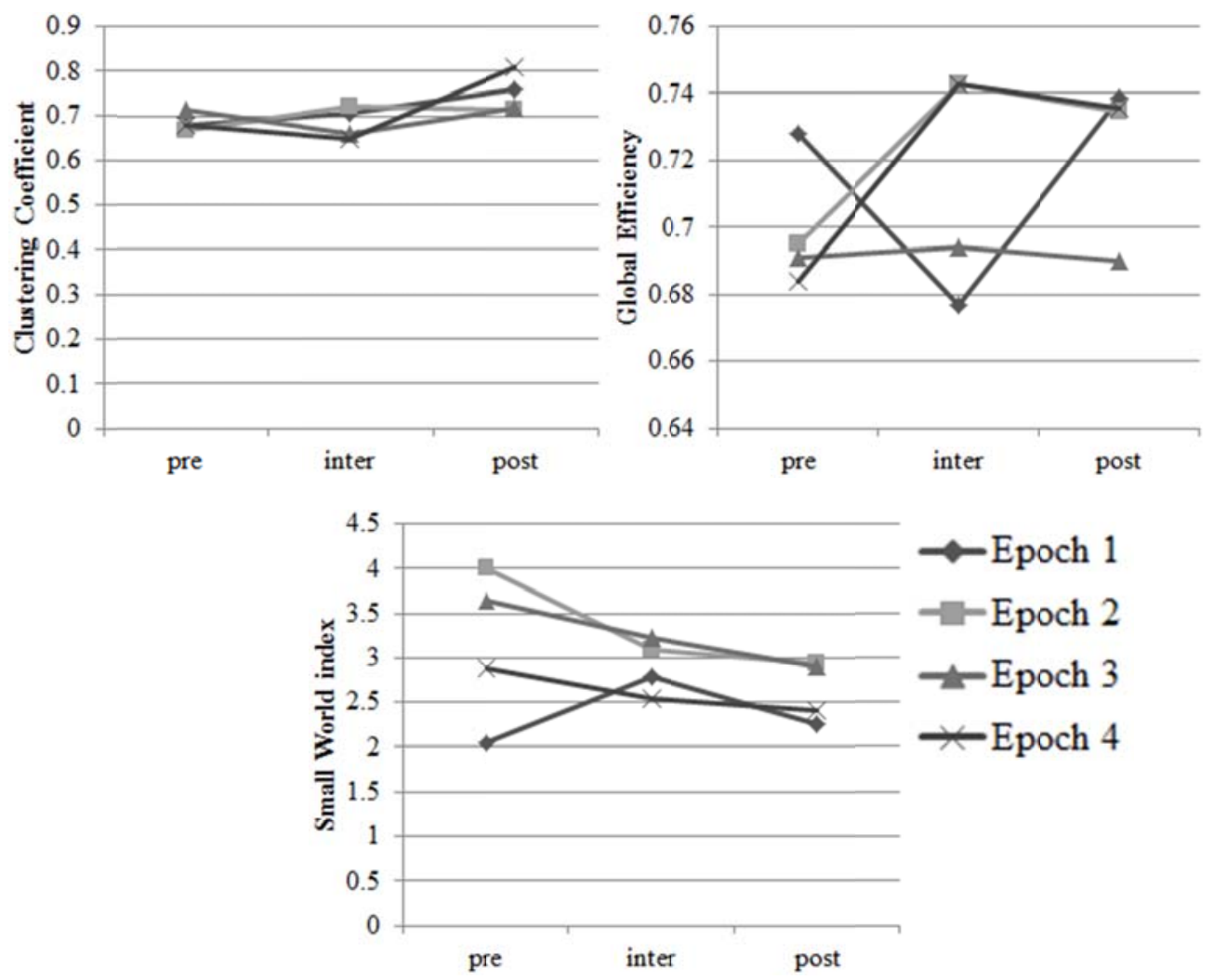

Figure 4.28: Graph Theory Measurements corresponding to pre- inter- post- ictal stages for different epochs across P14.

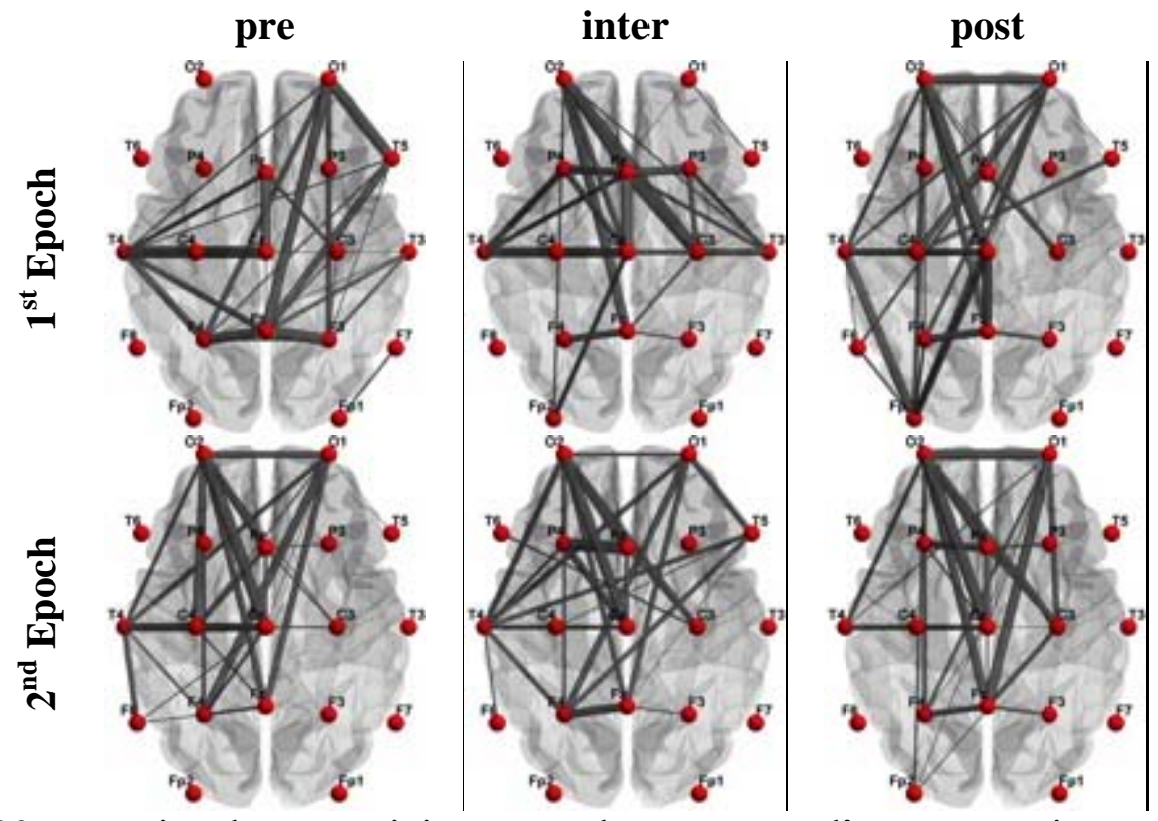

Figure 4.29: Functional connectivity networks corresponding to pre- inter- post- ictal stages for different epochs across for P15. 


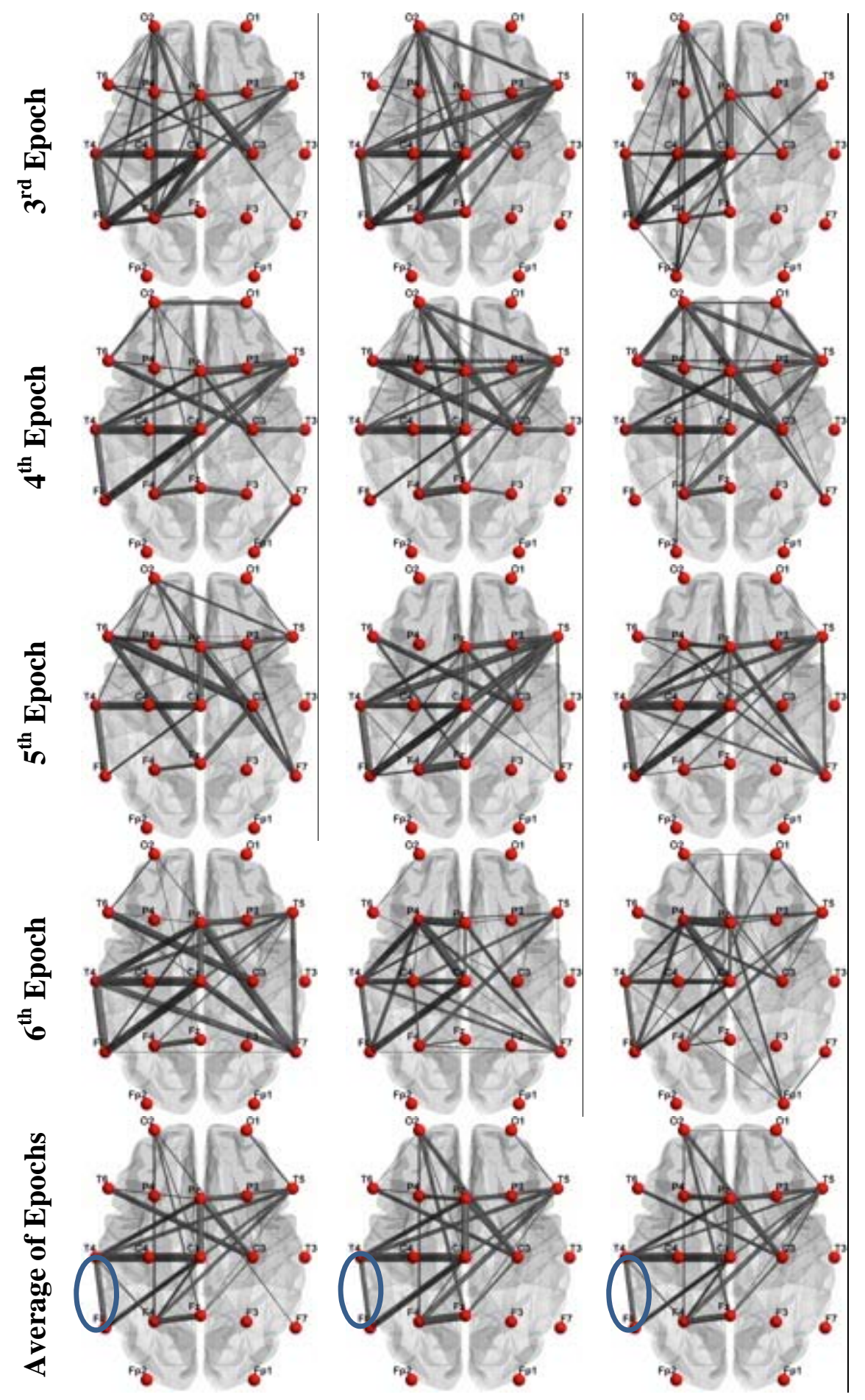

Figure 4.29 (cont.): Functional connectivity networks corresponding to pre- inter- postictal stages for different epochs across for P15. 

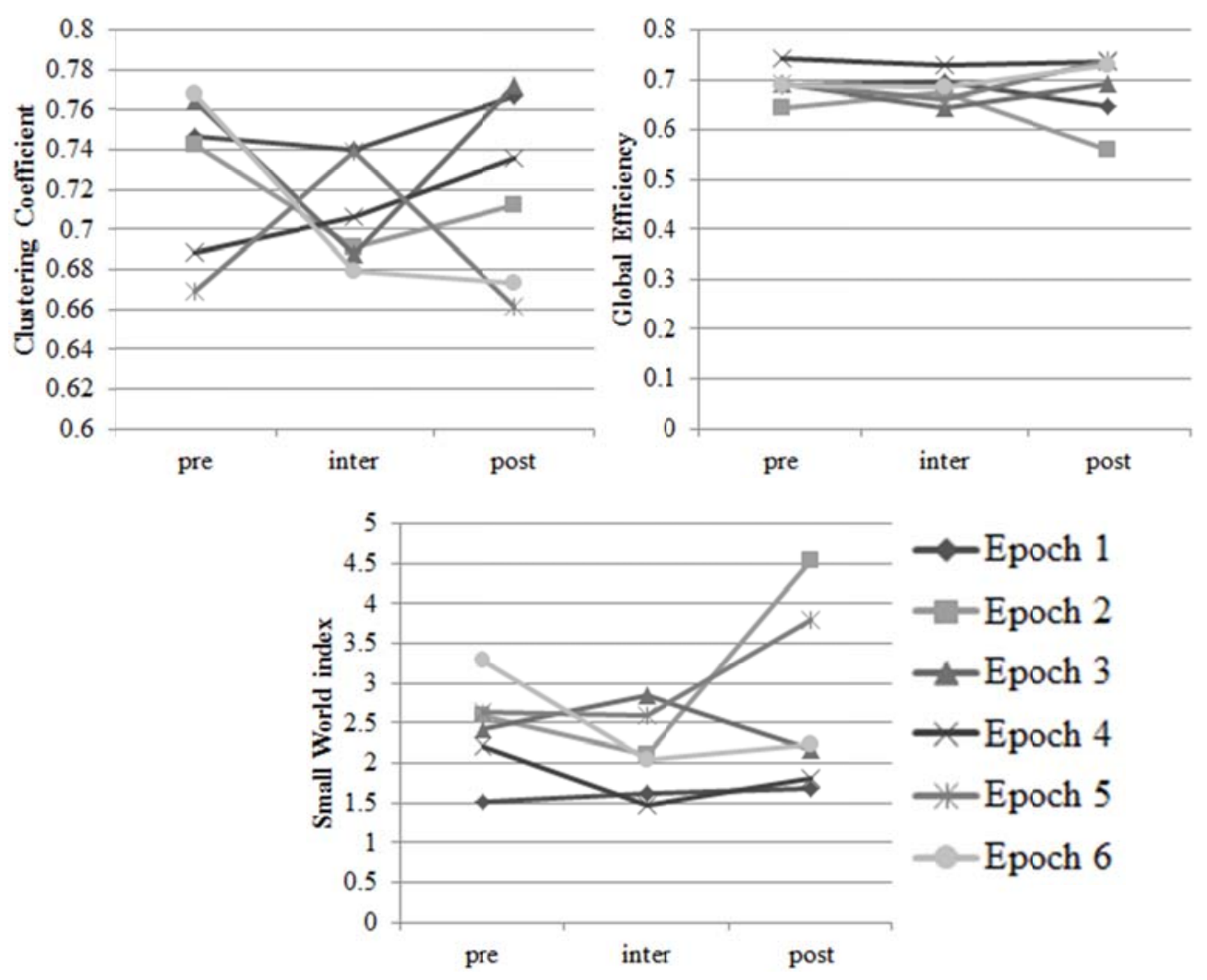

Figure 4.30: Graph Theory Measurements corresponding to pre- inter- post- ictal stages for different epochs across P15.
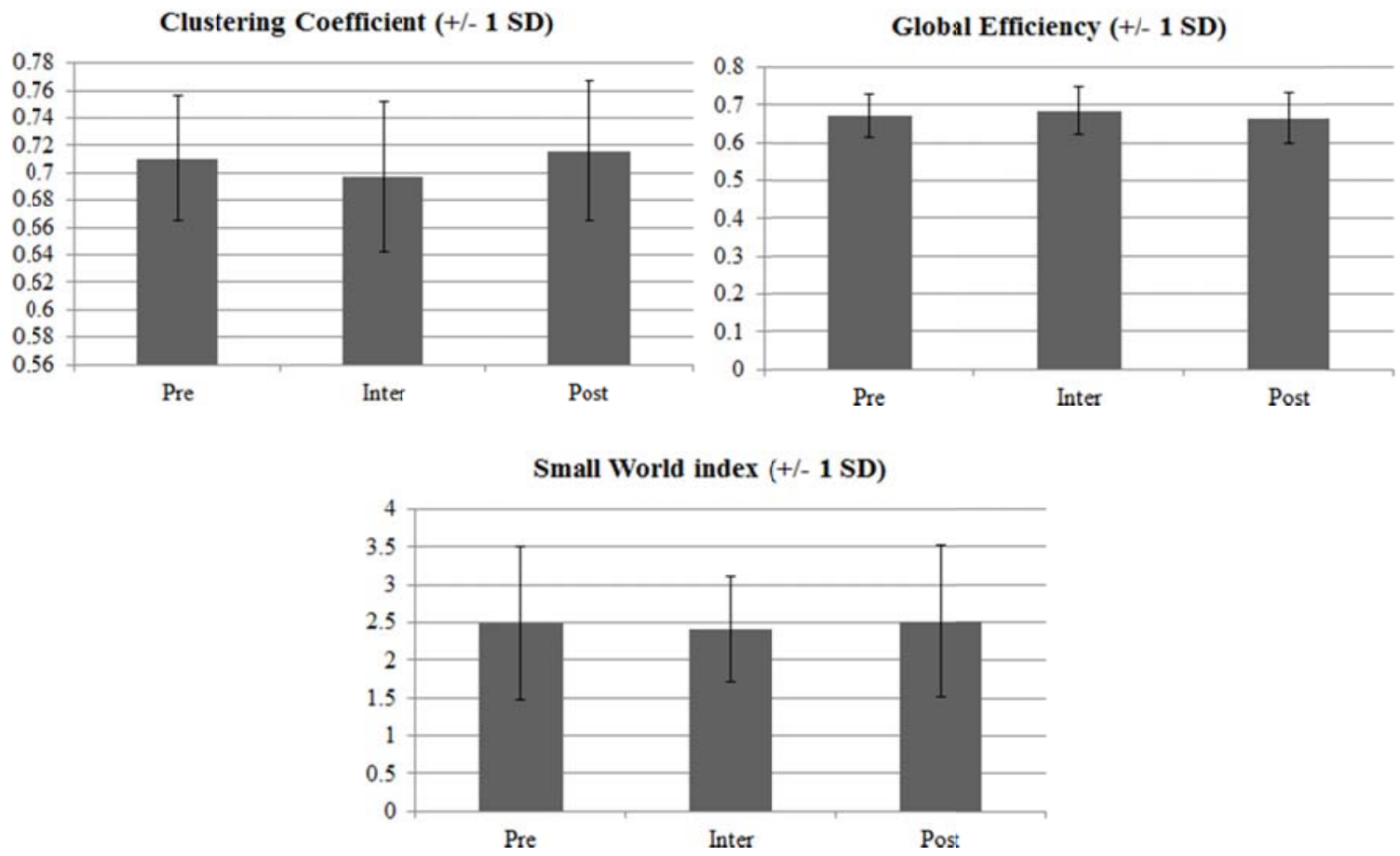

Figure 4.31: Mean and standard deviations of three network measures of graph theory based FCN's maps for different conditions (pre, inter and post). 
Table 4.2: Two-tailed paired t-test results ( $p$ values) of the means of each network measure across group for different stages of the evolution of interictal recordings

\begin{tabular}{llll}
\hline & pre vs. post & pre vs. inter & post vs. inter \\
\hline Clustering Coefficient & 0.50 & 0.09 & $<\mathbf{0 . 0 5}$ \\
Global Efficiency & 0.31 & $<\mathbf{0 . 0 5}$ & $<\mathbf{0 . 0 5}$ \\
Small World index & 0.88 & 0.56 & 0.48 \\
\hline
\end{tabular}

According to the test results, mean of global efficiency for pre condition is statistically different $(p<0.05)$ from inter condition. The same significant difference is found between the mean of global efficiency of post condition and the one of inter condition. Clustering coefficient of post condition is significantly $(p<0.05)$ different from the clustering coefficient of inter condition.

Clustering coefficient is an index of the likelihood of graph nodes to form a cluster together. Social networks are found to have higher clustering coefficient than random networks. Our group analysis of clustering coefficient across the networks showed that mean clustering coefficient over the interictal recordings (0.69) is less than the clustering coefficient for the pre-ictal condition (0.71) and the one for the post-ictal condition (0.71). Global efficiency measures the efficiency of a network in exchanging information across the network globe. Mean global efficiency of the networks for the interictal duration (0.68) is statistically greater than those of the pre-ictal condition $(0.67)$ and the post-ictal condition (0.66). Although small world index for the interictal recording (2.41) is not found to be significantly different than the indices for pre-ictal (2.49) and post-ictal (2.51), however, the networks in the interictal condition tend to be small world networks with lesser degree than the other two conditions. 


\subsection{Conclusion}

The proposed methodology was successfully utilized to construct and analyse the FCN's of interictal recordings for the patients with epilepsy. The efficiency of the methodology was evaluated in its capability to localize the focus and side of the epileptogenic source.

Graph theory based analysis of FCN's maps corresponding to pre- inter- and post-ictal recordings showed significant changes in the network measures such as clustering coefficient and global efficiency. 


\section{CHAPTER 5}

Intracranial Volume Estimation

\subsection{Introduction}

Intracranial volume (ICV) is a standard measure often used in morphometric analyses to correct for head size in brain studies. Inaccurate ICV estimation could introduce bias in the outcome. Chapter 5 of the current dissertation provides a decision aid in defining protocols for ICV estimation across different subject groups in terms of sampling frequencies that can be optimally used on the volumetric MRI data, and type of software most suitable for use in estimating the ICV measure. Four groups of 53 subjects are considered, including adult controls (AC), adults with Alzheimer's disease (AD), pediatric controls (PC) and group of pediatric epilepsy subjects (PE). Reference measurements were calculated for each subject by manually tracing intracranial cavity without sub-sampling. The reliability of reference measurements were assured through intra- and inter- variation analyses. Three publicly well-known software packages (Freesurfer Ver. 5.3.0, FSL Ver. 5.0, SPM8 and SPM12) were examined in their ability to automatically estimate ICV across the groups. The study assumes a priori knowledge about the population under study into the automated ICV estimation.

\subsection{Materials and Methods}

An overview of the study protocol, which includes two phases of analysis, is presented in Figure 5.1. Input to both phases of the study is the MRI T1-weighted image volume of the subject. Phase I of the study, which is two months leading to phase II of the study, involves reference manual measurement of ICV by Operator 1 (Op1). The estimated ICV measurements from Freesurfer, FSL and SPM are calculated and contrasted against the 
reference manual ICV and the errors $\Delta_{F S}^{1}, \Delta_{F S L}^{1}$ and $\Delta_{S P M}^{1}$ are calculated for Freesurfer, FSL and SPM, respectively. Second operator (Op2) is provided with the same T1weighted image volume of the subject and inter-operator variability analysis is performed. The MRI data were identical in both phases I and II of the study. Intraoperator variability analysis is conducted by re-measuring the ICV by Operator 1 in phase II of the study. Automatic measurements of ICV using FS, FSL and SPM are also repeated to compare the intra-software reproducibility. The second phase of the study was implemented with similar processing power to keep the results unbiased from the potential unbalanced processing units in software measurements.

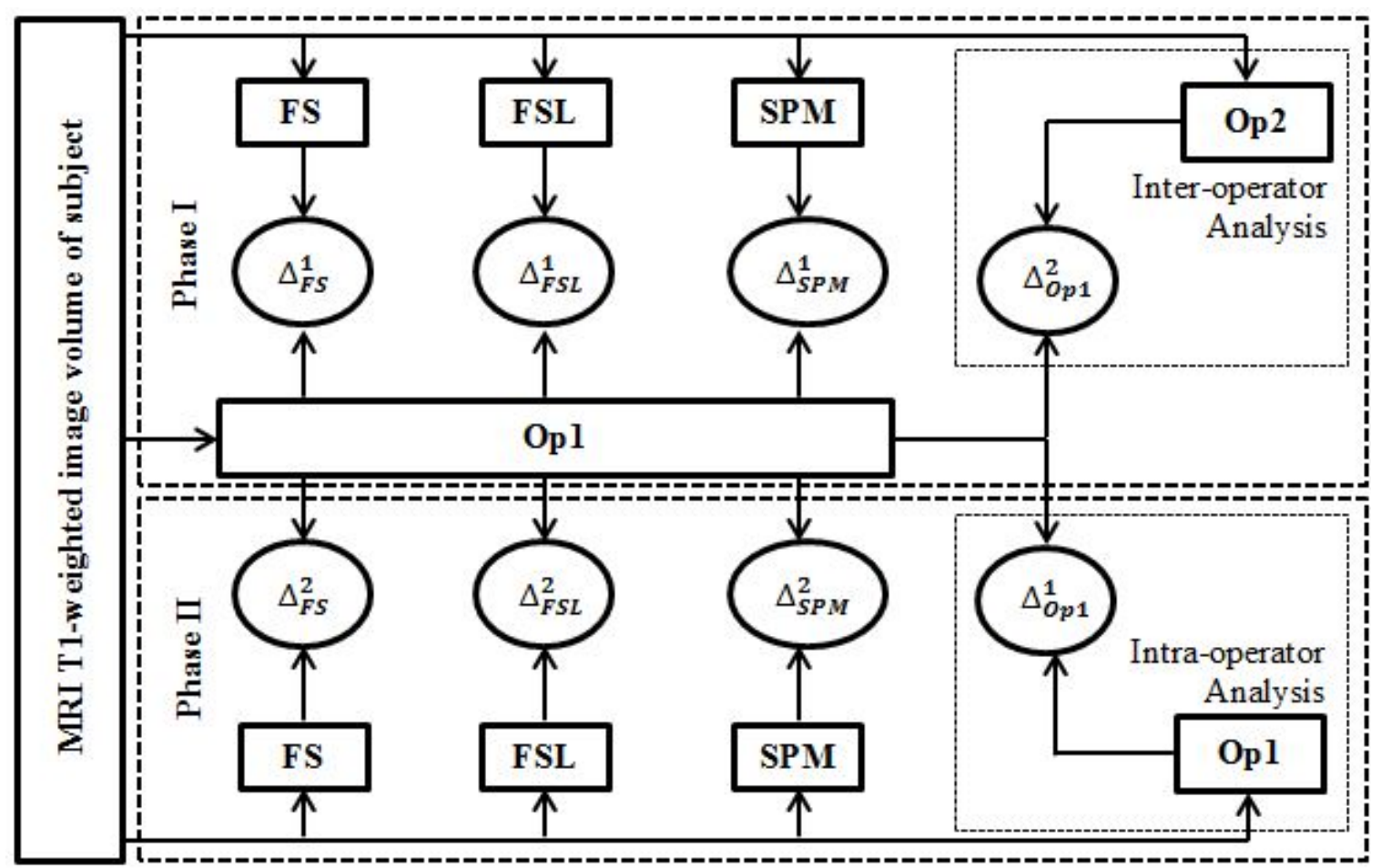

Figure 5.1: An overview of the study protocol. 
Table 5.1: Demographic characteristics of study subjects

\begin{tabular}{ccc}
\hline \hline & Age & Female/Male \\
\hline PE $(\mathbf{n}=\mathbf{1 3})$ & $13 \pm 2.93^{*}$ & $8 / 5$ \\
PC $(\mathbf{n}=\mathbf{1 8})$ & $11 \pm 1.26$ & $8 / 10$ \\
AD $(\mathbf{n}=\mathbf{1 1})$ & $81 \pm 9.31$ & $6 / 5$ \\
AC $(\mathbf{n = 1 1})$ & $71 \pm 6.21$ & $9 / 2$ \\
\hline
\end{tabular}

*Data presented as mean \pm standard deviation where applicable.

\subsubsection{Subjects and Images}

$\mathrm{AD}$ and $\mathrm{AC}$ groups subjects are from the Wien Center for Alzheimer's Disease and Memory Disorders with the Mount Sinai Medical Center, Miami Beach, FL, USA. Subjects from both $\mathrm{AD}$ and $\mathrm{AC}$ groups have taken the Folstein Mini-Mental State Examination (Folstein, Folstein et al. 1975) with a minimum score of 15 out of 30. AD and $\mathrm{AC}$ subjects had a neurological and medical evaluation by a physician acoording to the neuropsychological tests (Duara, Loewenstein et al. 2010).

MRI scans of the brain for Adult population groups, $\mathrm{AD}$ and $\mathrm{AC}$, were acquired on a 1.5T machine (Siemen's Symphony, Iselin, N.J., USA, or General Electric, HDX, Milwaukee, Wisc., USA) using a proprietary 3D-magnetization-prepared rapidacquisition gradient echo (MPRAGE). Specifications for MPRAGE include coronal sections with a $1.5 \mathrm{~mm}$ gap in thickness; section interval, $0.75 \mathrm{~mm}$; TR, $2190 \mathrm{~ms}$; TE, $4.38 \mathrm{~ms}$; TI, $1100 \mathrm{~ms}$; FA, 15; NEX, 1; matrix, $256 \times 256$; FOV, $260 \mathrm{~mm}$; bandwidth, $130 \mathrm{~Hz} /$ pixel; acquisition time, 9 minutes; phase-encoding direction, right to left. Structural MRI for pediatric subject groups, PE and PC, were collected by a multisite consortium and repository (Lahlou, Guillen et al. 2006)for pediatric epilepsy to study the 
effects of pediatric epilepsy on the functionality of the brain as well as on structural impacts (Guillen, Adjouadi et al. 2009; Eddin, Wang et al. 2014).

\subsubsection{Reference ICV Estimation}

Reference ICV measurements were performed for all 53 subjects from the four groups by operator 1, Op1, during phase I of the study. Op1 repeated the process of measuring ICV across all subjects during phase II of the study, in order to evaluate intra-operator variability. A second operator, Op2, calculated the ICV of all subjects during phase II of the study to assess the inter-operator variability in calculating ICV. No specific order of subjects/groups was considered by both operators when measuring ICV to lessen the possible learning bias across groups. No time limitation in reference ICV measurement was imposed on both operators, Op1 and Op2. A computer assisted approach, using an AFNI plugin(Cox 1996), was conducted by the operators to manually draw masks in every slice of the volume and highlighting voxels which belong to ICV.

Voxels were included in the ICV mask in each slice by strictly following the protocol from a recent study (Nordenskjöld, Malmberg et al. 2013). The delineation step was performed on the histogram equalized image for each slice. Histogram equalization is performed to enhance the contrast of T1-weighted images to improve visual clarity of the biomarkers. The ICV was measured by counting the total number of voxels highlighted as belonging to ICV multiplied by the voxel volume. Figure 5.2 shows a sample slice of T1-weighetd image volume corresponding to one randomly selected subject from each group with the same slice with ICV highlighted. Their histogram equalized images are shown in the middle column of Figure 5.2. Left column images are the original T1- 
weighted image sagittal slice; middle column is the histogram equalized version of the same image with arrows pointing to the dura; right column is the slice with ICV highlighted pixels.Arrows are pointing to the dura matter which is considered as a landmark in segmenting ICV from other brain tissues.

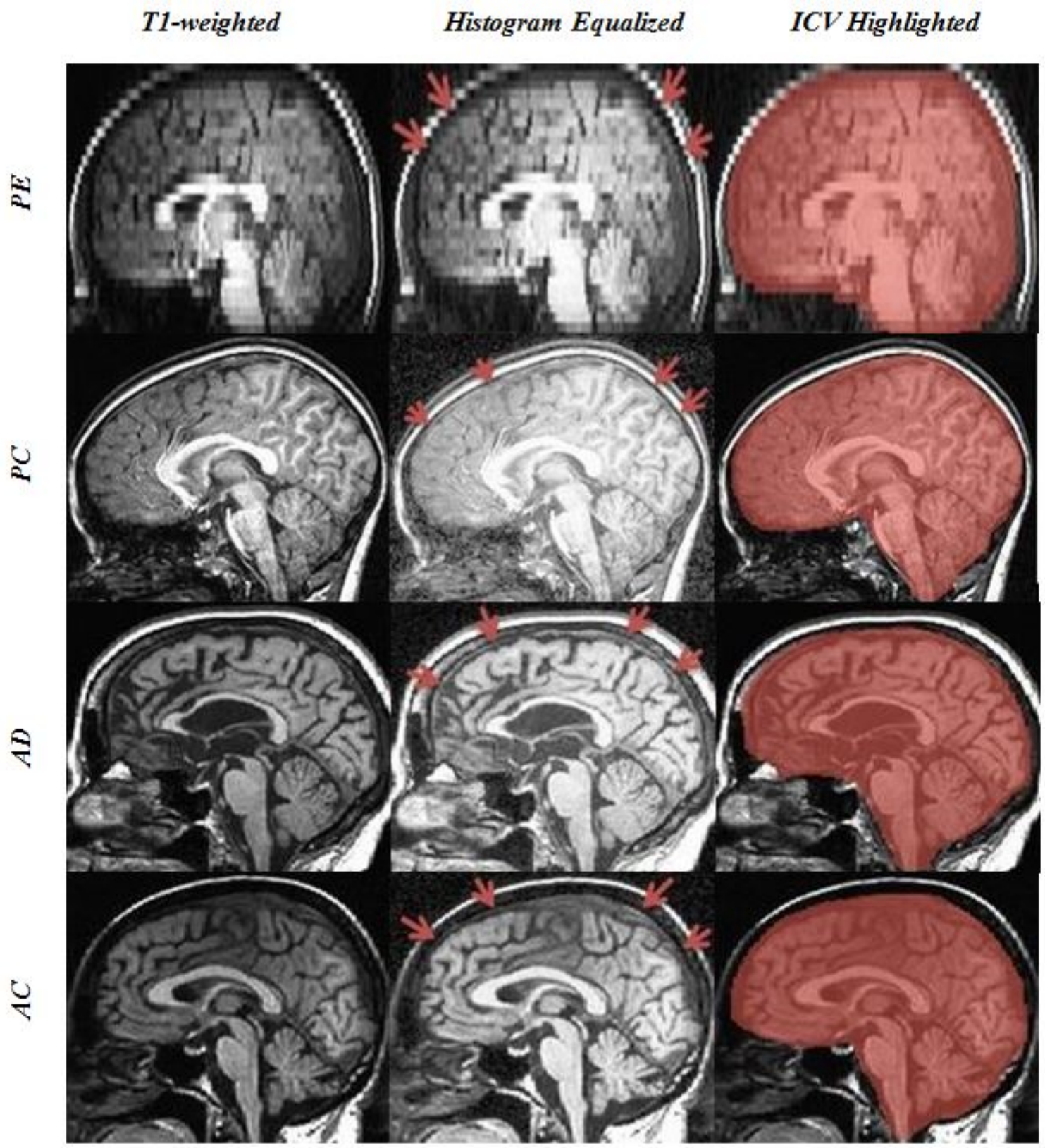

Figure 5.2: Reference ICV segmentation for a sample sagittal slice of randomly selected subject from each group. 


\subsubsection{Automatic ICV Estimation}

During phase I of the study, T1-weighetd image volumes of all subjects in four categories were subjected to ICV estimation through Freesurfer (FS 5.1.0), FSL (FSL 5.0) and Statistical Parametric Mapping (SPM 8). A set of default parameters were chosen when required by the software and no other external interventions were involved. The process of automatic ICV measurement using FS, FSL and SPM was repeated in phase II of the study to evaluate the intra-software variability.

FS (v5.3.0): T1-weighted image volumes of all subjects from four groups were processed through automatic image processing pipeline of Freesurfer software (https://surfer.nmr.mgh.harvard.edu). Estimated ICV reported as part of the statistics file (aseg.stats file) corresponding to the subject, created by the Freesurfer(Dale, Fischl et al. 1999), was recorded as the ICV estimation for the subject. ICV estimation by FS is an atlas-based estimation approach (Buckner 2004) which assumes that a correlation exists among ICV of a subject and determinant of the registration matrix used to transform the image from subject space to the atlas.

FSL 5.0: ENIGMA protocol (http://enigma.ini.usc.edu) was used to automatically estimate ICV using FSL (http://fsl.fmrib.ox.ac.uk). ICV estimation through FSL (Jenkinson, Pechaud et al. 2005) following the above mentioned protocol is also an atlas based estimation procedure where the subject image is first linearly aligned to MNI152 standard space. ICV is next calculated by multiplying the inverse of the determinant of the affine matrix by the size of the template brain. The protocol itself corrects for field bias with a two steps Brain Extraction Tool (BET) (Jenkinson, Pechaud et al. 2005) with an intermediate FAST (FMRIB's automated segmentation tool). The default parameter 
values of 0.5 and 0 were set respectively for fractional intensity threshold and threshold gradient.

SPM 8: VBM toolbox (http://www.fil.ion.ucl.ac.uk/spm) of Statistical Parametric Mapping (Ashburner and Friston 2000) was used with default parameters ("Non-linear only" modulation) to segments the voxels of T1-weighetd brain volume into four classes, namely white matter (WM), gray matter (GM), cerebrospinal fluid (CSF) and other. WM, GM and CSF volumes were summed up to provide an estimate of ICV. To attain the automatic feature of the method, no preprocessing or re-orientation were applied on the T1-weighetd images in advance to estimate the ICV (Bendel, Koivisto et al. 2010). However visual inspection of the images showed no major misalignment along the commissural line.

\subsubsection{Reliability Assessment of Manual ICV Estimation}

For each of the PE, PC, AD and AC groups, analysis of intra-operator variation, $\Delta_{o p 1}^{1}$, and inter-operator variation,,$\Delta_{o p 1}^{2}$, have been performed through paired t-test and correlation analysis.

\subsubsection{Statistical Analysis of Sampling Based Technique}

A randomized statistical testing procedure (Eritaia, Wood et al. 2000) was implemented to measure the accuracy of manual estimate of ICV by changing the sampling period for each group of subjects, PE, PC, AD and AC. Sampling period, $m$, is defined as the number of interleaved slices in tracing ICV across the brain volume, e.g. manual ICV estimation with a sampling period of 2 refers to tracing ICV in only half of the total number of slices. The larger the sampling period chosen, the less amount of time is 
required for ICV measurement. However, the accuracy of the measurement may drop with different rates for different subject categories. Different sampling periods, beginning from 2, were considered for ICV estimation. At each sampling period, $m$, ICV is calculated from a subset of slices. The first slice of the subset is the slice where the brain tissue appeared for the first time in the sequence of slices. The subsequent slices in the subset were selected every $m$ slices from the first slice until the brain tissue is no longer perceived. ICV of the subject was finally calculated as the sum of the traced volumes in the subset multiplied by $m$. The Intra-class correlation coefficient (ICC) among the reference ICV measurement and the estimated ICV at sampling frequency $m$ was calculated using two-way random ANOVA test. The process, initiated by randomly selecting the first slice of a given subset and ending with ICC calculation, was repeated five thousand times to create an empirical distribution of ICC's across each group to evaluate the effect of sampling period on the accuracy of the estimated ICV.

\subsubsection{Reliability Assessment of Automatic ICV Estimation}

For each of the $\mathrm{PE}, \mathrm{PC}, \mathrm{AD}$ and $\mathrm{AC}$ groups, three sets of criteria were evaluated to provide a decision aid in choosing automated tool(s) for ICV estimation: (1) Intrasoftware variations were assessed using paired t-test; (2) Across each subject group, the means of calculated ICV through each automated tool (FS, FSL and SPM) in phase I were tested against the mean of reference ICV measured by Op1, through post hoc t-tests under the general linear model, using the $\mathrm{R}$ package phia (http://CRAN.Rproject.org/package=phia); and (3) Mean related percentage of absolute difference (MRPAD) in ICV estimated by each automated tool within each subject group was calculated using equation (5.1). 


$$
M R P A D=\frac{1}{n} \sum_{i=1}^{n} \frac{\left|\Delta_{a u t_{i}}\right|}{I C V_{o 1_{i}}} \times 100
$$

where $\Delta_{a u t_{i}}$ is the error of the specific automated tool in ICV measurement from the reference measurement performed by Op1 in phase I; aut represents the automated tool employed: FS, FSL or SPM; and $n$ is the number of subjects within the group. Systematic bias as well as randomized error in automatic ICV estimation across each population group were calculated and reported to assess the reliability of each method that was tested.

\subsection{Results}

A summary of intra- and inter-operator variation analysis is presented in Table 5.2. High correlations (0.999) were observed for intra- and inter- operator measurements of ICV across PE, PC, AD and AC groups. Statistical tests as shown in Table 5.2 confirm the validity of the manual ICV measurements performed by Op1. ICV measurements performed by Op1 in phase I of the study were then considered as the reference measurements for the rest of the analysis.

Next was to study the effect of the sampling period on the reliability of manual estimation of ICV. For this purpose, random statistical testing procedure explained in section 5.2.4 was implemented on ICV measurements performed by Op1 (Phase I) for each subject group. The $5^{\text {th }}, 25^{\text {th }}, 50^{\text {th }}, 75^{\text {th }}$, and $95^{\text {th }}$ percentiles of the empirical distribution of ICC were calculated for each subject group, as shown in Figure 5.3, to contrast the estimated ICV based on a specific sampling period with the estimated ICV

considering the "every slice" protocol. Corresponding maximum percentage errors (MPE) were also given for each sampling period. The figure shows the randomness 
behavior of ICC value as the sampling period increases. ICCs are plotted for each group contrasting the estimated ICV based on a specific sampling rate with the estimated ICV considering every slice protocol; The right figures are Maximum Percentage Error plot for groups. ICC of 0.99 is highlighted in ICC plots and the corresponding sampling period is found as the point could be considered as a reference to not to lose the accuracy when using subsampling strategies.

Table 5.2: Intra- and inter- operator variation analysis for manual ICV estimation in different subject groups.

\begin{tabular}{llllllll}
\hline \hline & Op1 (Phase I) & Op2 (Phase I) & Op1 (Phase II) & $\Delta_{\mathbf{0 p 1}}^{\mathbf{1}}$ & $\Delta_{\mathbf{0 p 1}}^{2}$ & MRPAD $_{\mathbf{1}}$ & MRPAD $_{\mathbf{2}}$ \\
\hline PE & $1522.1 \pm 182.56^{*}$ & $1522.2 \pm 182.43$ & $1522.2 \pm 182.57$ & $\mathrm{p}=0.12$ & $\mathrm{p}=0.44$ & 0.002 & 0.011 \\
PC & $1640.1 \pm 179.56$ & $1640.2 \pm 179.63$ & $1640.1 \pm 179.56$ & $\mathrm{p}=0.61$ & $\mathrm{p}=0.10$ & 0.001 & 0.006 \\
AD & $1487.0 \pm 164.18$ & $1487.0 \pm 164.16$ & $1487.0 \pm 164.18$ & $\mathrm{p}=0.55$ & $\mathrm{p}=0.23$ & 0.002 & 0.004 \\
AC & $1460.9 \pm 144.44$ & $1461.0 \pm 144.36$ & $1460.9 \pm 144.46$ & $\mathrm{p}=0.35$ & $\mathrm{p}=0.33$ & 0.002 & 0.014
\end{tabular}

* $\overline{\mathrm{ICV}}(m L)$ presented as mean \pm standard deviation (a factor of $10^{6}$ has been taken from all values). Op 1 (Phase I): ICV measurements performed by Op1 in phase I of the study; Op2 (Phase I): ICV measurements performed by Op2 in phase I of the study; Op1 (Phase II): ICV measurements performed by Op1 in phase II of the study; $\Delta_{O p 1}^{1}$ is the result for testing the null hypothesis that the ICV measurements performed by Op1 (Phase I) is not significantly different from ICV measurements performed by Op1 (Phase II). $\Delta_{o p 1}^{2}$ is the result for testing the null hypothesis that ICV measurement calculated manually by Op1 (Phase I) is not significantly different from ICV measurements done by Op2 (Phase II). Paired t-test is used. $M R P A D_{1}$ : Mean related percentage of absolute difference between ICV measurements performed by Op1 in Phase I and Phase II which provides intra-operator error in manual ICV estimation. MRPAD ${ }_{2}$ : Mean related percentage of absolute difference between ICV measurements performed by Op1 and Op2 in Phase I which is related to inter-operator error.

The sampling periods and corresponding MPE values showed in dashed lines in Figure 5.3 correspond to the $95 \%$ probability of occurrence in keeping the reliability of the estimated ICV measurement above 0.99 based on sub-sampling protocols. Accordingly, a sampling period may not exceed $17.5 \mathrm{~mm}$ (with MPE ranging from 1.9 to 3.7) for PE; $27.5 \mathrm{~mm}$ (with MPE ranging from 0.6 to 2.9) for the PC group; $15.7 \mathrm{~mm}$ (with MPE 
ranging from 0.6 to 4.9) for the AD group; and $19 \mathrm{~mm}$ (with MPE ranging from 0.9 to 4.4) for the AC group.
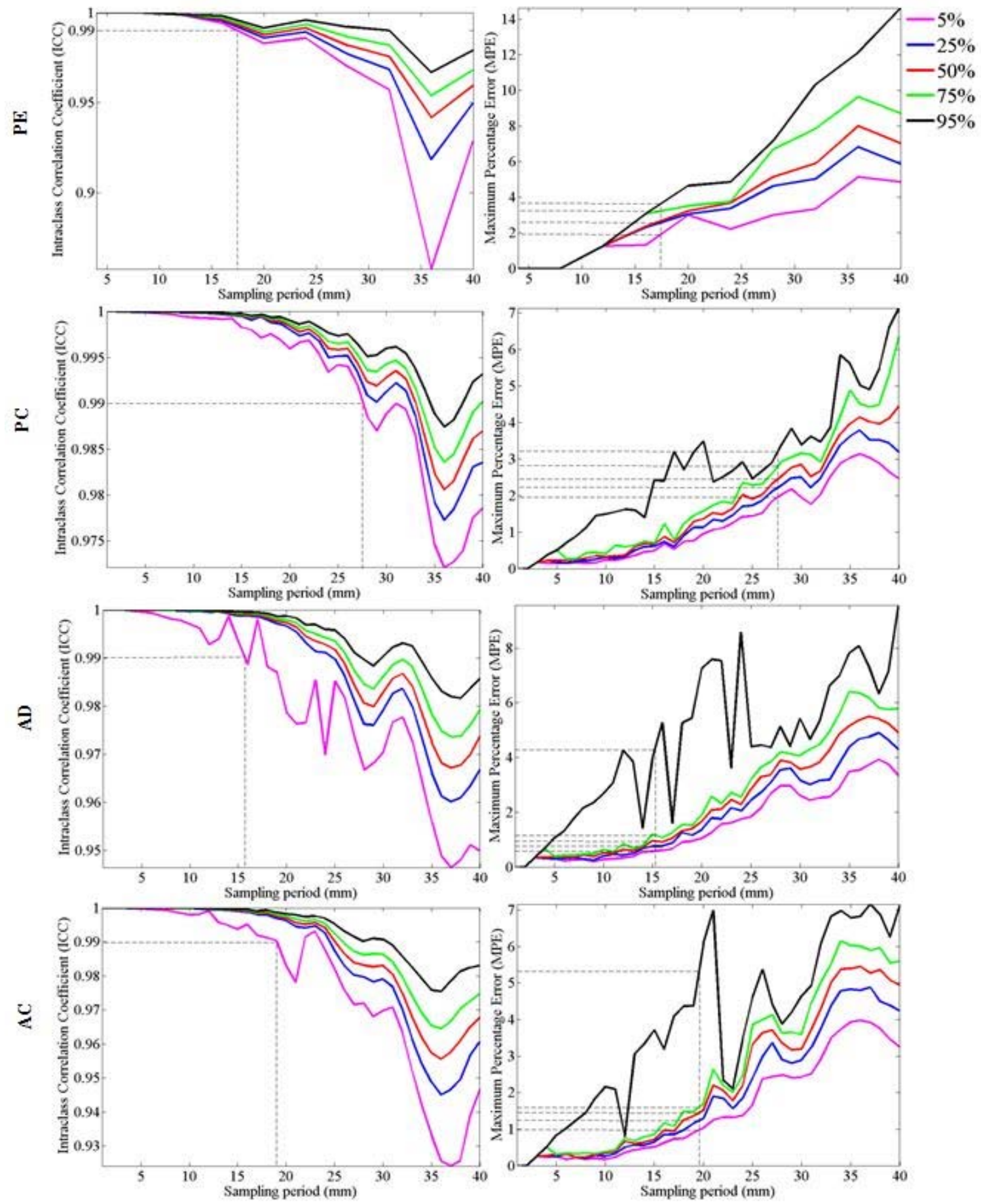

Figure 5.3: $5^{\text {th }}, 25^{\text {th }}, 50^{\text {th }}, 75^{\text {th }}$, and $95^{\text {th }}$ percentiles of the empirical distribution of intraclass correlation coefficient and maximum percentage error. 
Also, by defining the spread of empirical ICC distribution as the difference between the fifth percentile and $95^{\text {th }}$ percentile per sampling period, tight spread were considered as this difference to be lower than or equal with 0.005 (Eritaia, Wood et al. 2000). To keep a tighter spread, the sampling period should not exceed $20 \mathrm{~mm}$ for PE, $27 \mathrm{~mm}$ for PC, 10 $\mathrm{mm}$ for $\mathrm{AD}$ and $15 \mathrm{~mm}$ for $\mathrm{AC}$ group. The MPE shows the same random behavior with an incremental pattern as the sampling period gets higher.

The $90 \%$ confidence interval of MPE at sampling period of $40 \mathrm{~mm}$ is found to be within the range of $4 \%$ to $14 \%$ for $\mathrm{PE}, 2.5 \%$ to $7 \%$ for $\mathrm{PC}, 3 \%$ to $10 \%$ for $\mathrm{AD}$ and $3.5 \%$ to $7 \%$ for the AC group. These findings confirm the importance in carefully choosing an appropriate sampling period for the different subject groups when manual estimation of ICV is used. Interestingly, the results also show that a lesser number of slices is required to be segmented for ICV estimation in control groups ( $\mathrm{PC}$ and $\mathrm{AC}$ ) in contrast to the patient groups ( $\mathrm{PE}$ and $\mathrm{AD}$ ). This is also reflected in the range of the MPE variance where broader range of error is observed as the rate of the sampling period increases for $\mathrm{PE}$ and $\mathrm{AD}$ group as compared with their age matched control groups, $\mathrm{PC}$ and $\mathrm{AC}$.

To evaluate the effectiveness of FS, FSL and SPM in the automated ICV estimation process, ICV measurements through the select automated tools (FS, FSL and SPM) are plotted in Figure 5.4 against the ICV measurements performed by Op1 in phase I as well as Bland-Altman (B-A) plots (Martin Bland and Altman 1986) to evaluate the agreements between reference measurements with the automated ones. by Op1 in Phase I for each subject group (PE, PC, $\mathrm{AD}$ and $\mathrm{AC}$ ). The bold dashed line defines the reference line, and the solid lines are the regression lines of the ICV estimated by each of the automated 
tools used. Corresponding B-A plots where dashed line are highlighting 95\% limits for each automated tool are shown in the right side. Within each subject group (PE, PC, AD and AC), paired t-test statistics of the difference between each automated tool's ICV estimations and the reference ICV estimations across each group along with correlation coefficient and the corresponding MRPAD values are given in Table 5.3. Intra-software variation analysis is performed across each subject group. Table 5.4 summarizes the intra-software variation. Box plots are shown in Figure 5.5.

Table 5.3: Software reliability analysis for automated ICV estimation versus reference estimation (Op1)

\begin{tabular}{|c|c|c|c|c|c|c|c|c|c|c|}
\hline \multicolumn{6}{|c|}{$\mathrm{PE}$} & \multicolumn{5}{|c|}{ PC } \\
\hline & $\mathbf{p}$ & $\mathbf{r}$ & MRPAD & $\begin{array}{c}\text { Systematic } \\
\text { Bias }\end{array}$ & $\begin{array}{c}\text { Randomized } \\
\text { Error }\end{array}$ & $\mathbf{p}$ & $\mathbf{r}$ & MRPAD & $\begin{array}{c}\text { Systematic } \\
\text { Bias }\end{array}$ & $\begin{array}{c}\text { Randomized } \\
\text { Error }\end{array}$ \\
\hline FS & $<0.01$ & 0.51 & 43.9 & 862.27 & $\begin{array}{c}671.44 \\
\pm 317.91\end{array}$ & $<0.01$ & 0.87 & 30.3 & 214.04 & $499 \pm 184.83$ \\
\hline FSL & 0.26 & 0.73 & 13.41 & -250.13 & $\begin{array}{c}211.57 \pm 242.6 \\
3\end{array}$ & $<0.01$ & 0.98 & 11.35 & -317.77 & $\begin{array}{c}189.32 \pm 120.1 \\
8\end{array}$ \\
\hline $\begin{array}{l}\text { SPM } \\
8\end{array}$ & $<0.01$ & 0.45 & 16.6 & 587.63 & $\begin{array}{c}263.15 \pm 329.5 \\
4\end{array}$ & $<0.01$ & 0.45 & 20.3 & 1081.6 & $\begin{array}{c}339.77 \pm 258.1 \\
3\end{array}$ \\
\hline \multicolumn{6}{|c|}{$\overline{A D}$} & \multicolumn{5}{|c|}{ AC } \\
\hline & $p$ & $r$ & $M R P A D$ & $\begin{array}{c}\text { Systematic } \\
\text { Bias }\end{array}$ & $\begin{array}{c}\text { Randomized } \\
\text { Error }\end{array}$ & $p$ & $r$ & $M R P A D$ & $\begin{array}{l}\text { Systematic } \\
\text { Bias }\end{array}$ & $\begin{array}{c}\text { Randomized } \\
\text { Error }\end{array}$ \\
\hline FS & 0.1 & 0.68 & 9.6 & 978.42 & $\begin{array}{c}139.63 \pm 422.4 \\
5\end{array}$ & 0.13 & 0.68 & 5.4 & 281.77 & $82.69 \pm 168.49$ \\
\hline FSL & $<0.01$ & 0.67 & 91.80 & 1312.17 & $\begin{array}{c}704.18 \pm 997.4 \\
7\end{array}$ & $<0.01$ & -0.2 & 41.74 & 1445.31 & $\begin{array}{c}591.79 \pm 790.6 \\
0\end{array}$ \\
\hline $\begin{array}{l}\text { SPM } \\
8\end{array}$ & $<0.01$ & 0.48 & 12.5 & 644.57 & $\begin{array}{c}189.27 \pm 165.0 \\
2\end{array}$ & $<0.01$ & 0.82 & 10.1 & 37.84 & $\begin{array}{c}151.76 \pm 167.4 \\
3\end{array}$ \\
\hline
\end{tabular}

PE: Pediatric Epilepsy subjects group; PC: Pediatric Control subjects group; AD: Adult With Alzheimer's Disease subject groups; AC: Adult Control subject groups; Systematic Bias and Randomized errors are given in $m L$ unit. Randomized Error is presented in the form of mean $\pm 2 *$ standard deviation; $p$ : Paired ttest $\mathrm{p}$-value; $r$ : correlation coefficient; MRPAD: Mean Related Percentage of Absolute Difference; $\mathrm{p}$-value less than 0.01 was considered significant. 

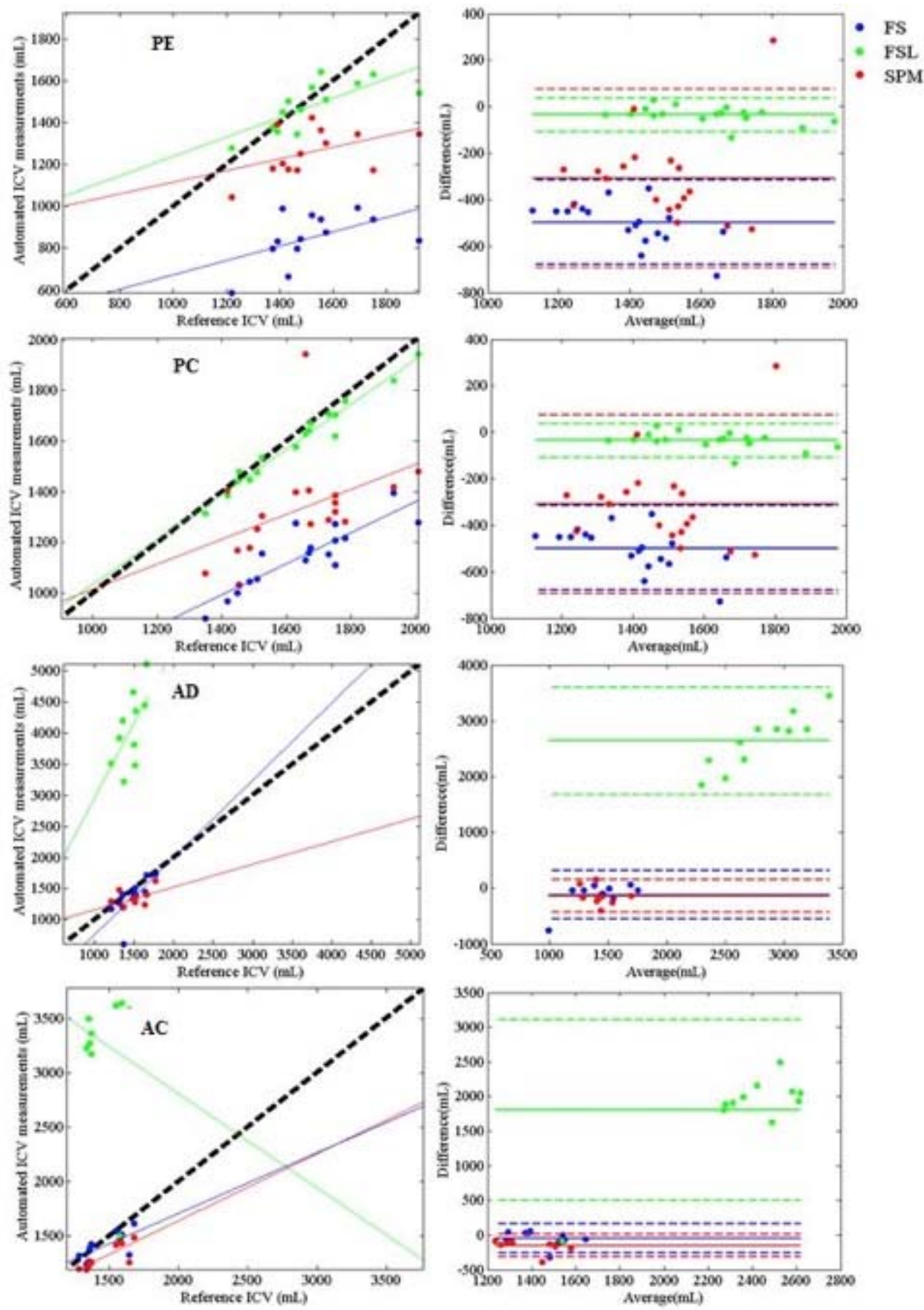

Figure 5.4. Plot of ICV measurements through automated tools (FS, FSL and SPM) against ICV measurements performed 
Table 5.4: Intra- software (phase I and phase II) variation analysis for automated ICV estimation

\begin{tabular}{|c|c|c|c|c|c|c|c|c|c|c|c|c|}
\hline & & PE & & & PC & & & AD & & & AC & \\
\hline & $\mathbf{p}$ & $\mathbf{r}$ & MRPAD & $\mathbf{p}$ & $\mathbf{r}$ & MRPAD & $\mathbf{p}$ & $\mathbf{r}$ & MRPAD & $\mathbf{p}$ & $\mathbf{r}$ & MRPAD \\
\hline FS & $<0.01$ & -0.78 & 94.71 & $<0.01$ & 0.47 & 32.88 & 0.25 & 0.71 & 11.52 & $<0.01$ & 0.99 & 1.09 \\
\hline FSL & $<0.01$ & 0.98 & 4.62 & $<0.01$ & 0.99 & 2.46 & $<0.01$ & 0.82 & 25.46 & $<0.01$ & 0.47 & 33.73 \\
\hline SPM & 0.99 & 0.61 & 4.05 & $<0.01$ & 0.82 & 7.97 & 0.49 & 0.63 & 5.96 & 0.1 & 0.99 & 0.5 \\
\hline
\end{tabular}

$p$ : paired t-test p-value; $r$ : correlation coefficient; MRPAD: Mean Related Percentage of Absolute Difference; $p$-value less than 0.01 was considered significant.
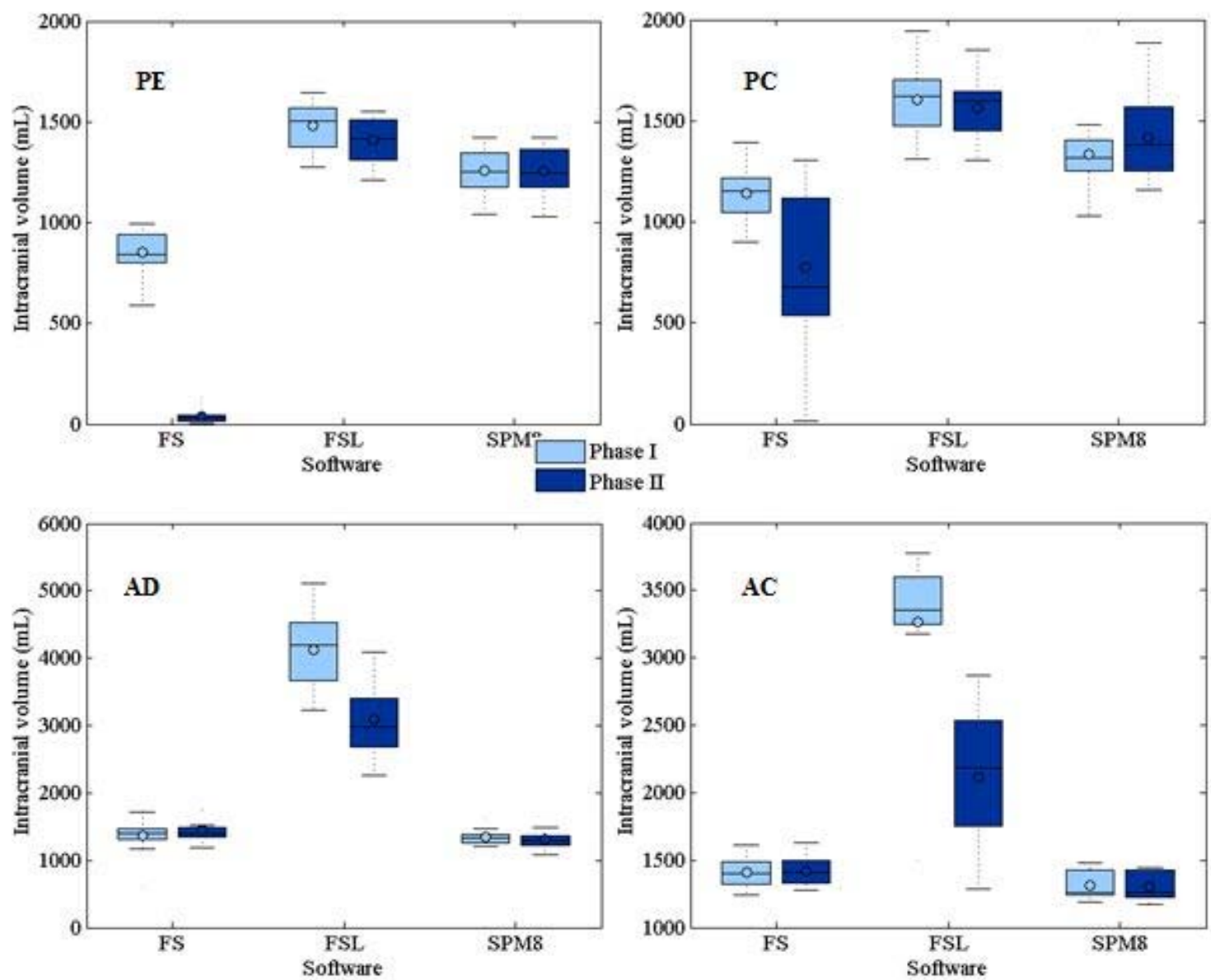

Figure 5.5: Intra-software variations in automated ICV measurement of each software (FS, FSL and SPM) are shown as box plots in Phase I of the study and Phase II of the study across each subject group (PE, PC, AD and AC). 


\subsection{Conclusion}

In retrospect, this chapter emphasized the importance in selecting the appropriate protocol in the automated estimation of ICV and the choice of the sampling period in the manual estimation of ICV, which are shown to depend largely on the demographics of the targeted population, the imaging parameters of the MR machine, as well as the neurological disorder under study. The contributions in this Chapter relate to establishing guidelines for the proper setting of the parameters involved in the process of ICV estimation. A retrospective on the results suggest the following findings for each of the study groups:

PE Subject Group: FSL is found to be more reliable in automatic ICV estimation across the PE group yielding the highest correlation (0.73) and lowest MRPAD value (13.41) in comparison to both FS and SPM which underestimated the reference ICV significantly $(\mathrm{p}<0.01)$. The minimum systematic bias $(-194.56 \mathrm{~mL})$ and randomized error $(87.99 \pm 191.79)$ in automatic ICV estimation were also observed when using FSL. Intrasoftware analysis across the PE group reflects effectiveness of FSL in automatic ICV estimation with the highest correlation coefficient (0.98) and lowest MRPAD (4.62); however, there is a significant difference $(p<0.01)$ between the means of FSL based ICV estimation between Phase I and Phase II.

PC Subject Group: FSL estimation of ICV has the highest correlation (0.98) with respect to the reference ICV and lowest MRPAD value (11.35), which shows the effectiveness of FSL in automatic ICV estimation for the PC group. However, all three software underestimated ICV $(\mathrm{p}<0.0 .1)$ at different levels, with FSL results being the 
closest one to the reference measurements. Systematic bias $(-96 \mathrm{~mL})$ and randomized error (40.21 \pm 62.23$)$ were minimum by using FSL to estimate ICV in PC group. Intrasoftware variation also supports the recommendation of using FSL for this PC group where highest correlation (0.99) and lowest MRPAD (2.46) are obtained as compared to the other two software tools (FS and SPM).

AD Subject group: FS showed to be an accurate tool for automatic ICV estimation across AD subject group where a high correlation (0.68) is found among automatic ICV measurements and the reference ICV. MRPAD of FS across AD subject group is found to be 9.6. FS is found to be a reliable tool for AD group as the intra-software variation of FS is found to be non-significant $(\mathrm{p}=0.25)$ and the correlation is ranked second (0.71). SPM is also showed to be a suitable candidate when choosing an automated tool for ICV estimation for the AD group with MRPAD value of 12.5. SPM showed a lesser systematic bias of $644.57 \mathrm{~mL}$ when compared with FS $(978.42 \mathrm{~mL})$ but with a larger randomized error than FS. However, FSL is found to upwardly bias $(p<0.01)$ the ICV measurements for AD causing a very high MRPAD value (91.8) as well as a significant variation for its intra-software variation.

AC Subject group: Both FS and SPM provide competitive results in automatic ICV estimation with MRPAD of 5.4 and 10.1 respectively, which makes them both good candidates when choosing an automatic tool for ICV estimation across the AC groups. SPM has a very low systematic bias compared to FS but has a larger randomized error. However, the mean of ICV estimated of ICV using SPM is different $(\mathrm{p}<0.01)$ from the one of reference ICV measurement. This results in underestimation of SPM in ICV 
measurement. SPM shows more reliable (MRPAD value of 0.5) in intra-software variability analysis as compared with FS (MRPAD value of 1.09). FSL is not providing accurate results (correlation coefficient equal with -0.2 and MRPAD of 41.74) for ICV estimation across AC subject group. 


\section{CHAPTER 6}

Conclusions and Recommendations for Future Work

\subsection{Conclusions}

This dissertation addressed two important research themes in brain studies: 1) Brain functional connectivity network construction and analysis; and 2) Intracranial volume estimation. For the first research theme, a data-driven approach for constructing brain functional connectivity networks based on scalp EEG recordings was introduced in Chapter 3. Findings suggest that functional connectivity networks of pediatric epilepsy subjects using scalp EEG recordings are found to be statistically different from normal controls. Graph theoretical analysis of functional networks and their application in pediatric epilepsy diagnosis were discussed in the same chapter.

Chapter 4 evaluated the efficiency of the proposed methodology in time-varying analysis of the functional brain connectivity networks during interictal recordings of patients with severe forms of epilepsy. Graph theory measures of networks for the periods of pre-ictal, interictal and post-ictal were tested for their differences in the same chapter. Global efficiency and clustering coefficient are found to be statistically different for the interictal period. Chapter 5 discussed the manual and automatic estimation of ICV across different populations. The choice of the right protocol in ICV estimation of different populations found to have a statistically significant impact on the accuracy of measurement. Recommendations were given to choose the right protocol for accurate ICV estimation purposes. 


\subsection{Discussions}

\subsubsection{FCNs Construction and Analysis}

Novel decision support systems, like the ones presented in this dissertation, for computer aided diagnosis of pediatric epilepsy using machine learning techniques, enhance our understanding of the disease and help increase the chances of cure and recovery. The approach taken in the proposed system was based on constructing functional connectivity networks (FCNs) of the brain and analyzing graph theoretical-based features to identify the wiring pattern differences among pediatric control (PC) and pediatric epilepsy (PE) groups. The system is designed in such a way as to provide the clinicians with an initial screening result about the likelihood of a given subject to be epileptic or not.

The time-varying FCN's implementation increases the resolution by segmenting the multichannel EEG. This created the potential of diagnosing epilepsy without the need for long EEG recording session and time-consuming visual inspection, as conventionally employed. The main contribution of the study is the reliance of the algorithm on machine learning techniques to facilitate the screening process of potential epileptic patients without the need of a priori knowledge and without need for a training phase. The suggested window length in constructing FCNs, number of principal components (dimension of GMM) and the inspection of the possible causal relationships among cortical brain regions are the areas that need further investigation on a larger dataset.

The methodology for constructing brain functional connectivity network in this study could be extended for its potential applicability in identifying different types of epilepsy. Examples of such unique connectivity patterns are associated to the clinical aspects of the 
different type of seizures, as illustrated in Figure 6.1. We investigated more individual patients in terms of the correspondence of their functional connectivity maps with the source and side of the foci, however more thorough research needs to be followed to generalize the applicability.

(a)

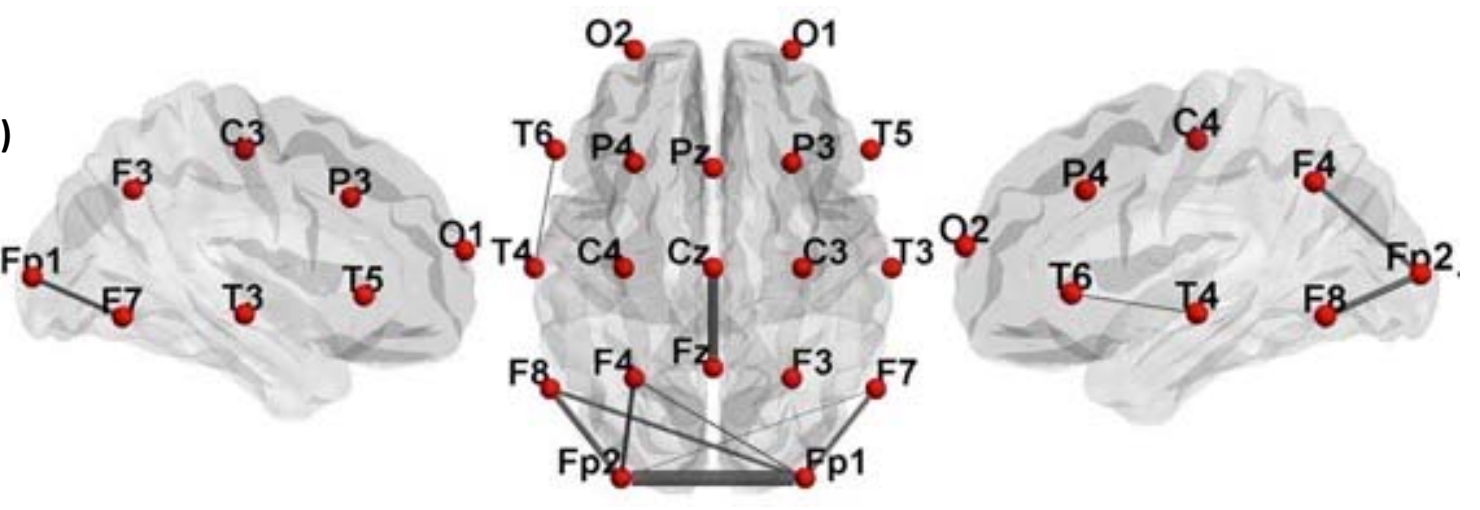

(b)
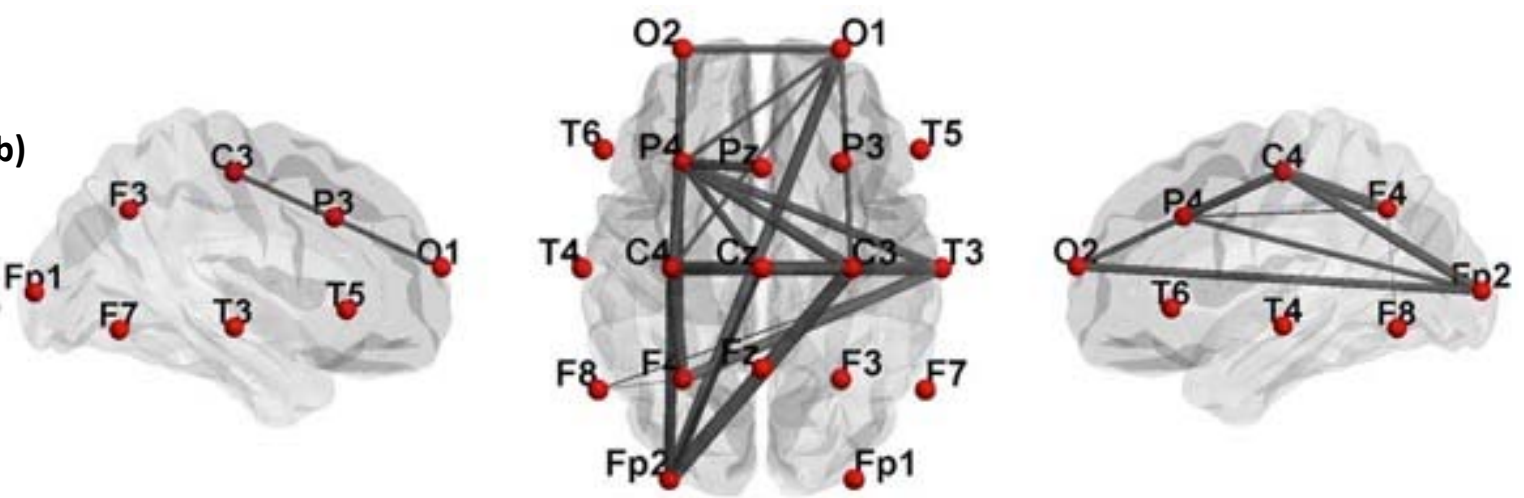

Figure 6.1: Constructed functional connectivity map (The threshold of $45^{\circ}$ is applied as the connection strength) for (a) subject diagnosed with left frontal region epilepsy and (b) subject diagnosed with generalized epilepsy.

Initial assessment of the applicability of the algorithm in identifying different types of epilepsy showed the ability to cluster generalized epilepsy from focal epilepsy; however, a more thorough investigation is needed to confirm the association of key characteristics in the connectivity patterns in direct relation to the type of seizure, 3D source 
localization, and potentially the connectivity in the structural MRI for the different regions of the brain.

\subsubsection{Intracranial Volume Estimation}

Chapter 5 of the dissertation provided a decision-making process as a guide for estimating ICV either manually or automatically, given the critical importance of ICV as a metric in brain volumetric studies in reference to several neurological disorders. Among them epilepsy and Alzheimer were reflected in this study. The main findings could be summarized in three very important points: (1) The choice of the software should take into consideration whether the population under study is pediatric or adult, with the knowledge that atlas-based software platforms such as FS and SPM tend to be more accurate when dealing with the adult population, since the atlas used in both is derived from an adult population; while FSL, free of the atlas bias, performs better with the pediatric population; (2) the sampling period, in terms of the number of slices that are considered, should be carefully evaluated in terms of the ICC value or accuracy in the ICV estimation, in order to overcome the heavy computational requirements when considering all the slices together with the burden imposed in the tediousness of the manual segmentation of ICV; and (3) default parameters are not necessarily the optimal parameters that should be used in the study.

In assessing the merits in considering the aforementioned 3 points, four groups of subjects with different neurological conditions, namely epilepsy and Alzheimer's disease, were considered: PE: Pediatric Epilepsy; PC: Pediatric Control; AD: Adult With Alzheimer's Disease; AC: Adult Control. Two operators performed the manual ICV 
measurements for all the subjects; one of them repeated the measurements in two phases to assess the intra-operator variance. The reliability of reference ICV measurements was assured for intra-operator and inter-operator variations. No statistical significant difference (MRPAD less than 0.01\%) was found across the subject groups considered. T1-weighted images provide low contrast between fluids and bone, which makes the process of ICV estimation prone to error. Studies (Pengas, Pereira et al. 2009; Ambarki, Lindqvist et al. 2012; Nordenskjöld, Malmberg et al. 2013) have overcome this limitation utilizing T2-weighted or PD images to estimate the reference ICV. While T1-weighted images suffer from the low contrast, brightness adjustment could help improve the visual clarity of ICV borders (Keihaninejad, Heckemann et al. 2010). The current study was enforced to estimate the reference ICV from T1-weighted images due to the absence of other recommended weighted signals (PD and T2), however, the histogram equalization technique helped to improve the contrast and facilitate the reference estimation. The histogram equalization technique (Ziaei, Yeganeh et al. 2008) reshapes the distributions of the intensities in an image and results in a higher contrast image. Although the brightness adjustment solutions could help improve the contrast, utilization of $\mathrm{T} 2$ weighted or PD images remains the preferred modalities (Nordenskjöld 2014).

Main factor analysis were performed in the adult population since imaging parameters were the same. Group-ID (AC, AD), Sex (Male, Female), Method (manual, FS, FSL, and SPM) and Age were considered as the main factors of this study. Analyzing their main effects and interactions on measured ICV showed that there is significant overall GroupID effect $(p<0.01)$, Sex effect $(p<0.01)$ and Method $(p<0.01)$. Furthermore, Interaction between Group-ID and Method factors was the only interaction found to be 
statistically significant $(p<0.01)$. Table 6.1 provides all the p-values for the different factors and the interaction between them.

Table 6.1: Main factors analysis and their interactions on the measured ICV.

\begin{tabular}{lll}
\hline & $\mathrm{F}$ & $\operatorname{Pr}(>\mathrm{F})$ \\
\hline Group-ID & $4.057815 \mathrm{e}+00$ & $6.359433 \mathrm{e}-02$ \\
Sex & $8.929189 \mathrm{e}+00$ & $9.777993 \mathrm{e}-03$ \\
Age & $1.069814 \mathrm{e}-01$ & $7.484464 \mathrm{e}-01$ \\
Method & $2.096274 \mathrm{e}+02$ & $2.732017 \mathrm{e}-25$ \\
Group-ID : Sex & $8.929189 \mathrm{e}+00$ & $9.777993 \mathrm{e}-03$ \\
Group-ID : Age & $5.299599 \mathrm{e}-03$ & $9.429964 \mathrm{e}-01$ \\
Sex : Age & $2.656019 \mathrm{e}-01$ & $6.143387 \mathrm{e}-01$ \\
Group-ID : Method & $8.024328 \mathrm{e}+00$ & $2.432221 \mathrm{e}-04$ \\
Sex : Method & $8.291057 \mathrm{e}-01$ & $4.853501 \mathrm{e}-01$ \\
Age : Method & $1.981851 \mathrm{e}-02$ & $9.961500 \mathrm{e}-01$ \\
Group-ID : Sex : Age & $1.012292 \mathrm{e}-03$ & $9.750675 \mathrm{e}-01$ \\
Group-ID : Sex : Method & $6.211696 \mathrm{e}-01$ & $6.052420 \mathrm{e}-01$ \\
Group-ID : Age : Method & $2.471838 \mathrm{e}-01$ & $8.628723 \mathrm{e}-01$ \\
Sex : Age : Method & $4.724365 \mathrm{e}-01$ & $7.031207 \mathrm{e}-01$ \\
Group-ID : Sex : Age : Method & $5.051764 \mathrm{e}-01$ & $6.808114 \mathrm{e}-01$ \\
\hline
\end{tabular}

In the case of manual estimation of ICV, the study showed that there is causality between the accuracy and reliability of the measured ICV with respect to the number of slices considered in the segmentation process. The same finding was reported in a previous study using a group of adult control subjects(Eritaia, Wood et al. 2000), however, the current study reports the existence of a similar relationship between the number of slices considered for ICV segmentation and the reliability of the calculated ICV measurement across different subject groups, including both adult and pediatric populations. More importantly, the study also showed that the reliability of ICV measurements should be weighed across the different $\mathrm{PE}, \mathrm{PC}, \mathrm{AD}$ and $\mathrm{AC}$ groups, and that a set of guidelines should be considered when performing either manual or automatic ICV estimation procedures in terms of both the population under study and the software platform that is used. Consequently, the results shown earlier in Figure 5.3 could be utilized as a guide in 
choosing the right sampling period in manual ICV estimation. The current study finds that in order to keep the reliability of the estimated ICV measurement above 0.99 based on sub-sampling protocols, the sampling period may not exceed $17.5 \mathrm{~mm}$ for PE; 27.5 $\mathrm{mm}$ for PC group; $15.7 \mathrm{~mm}$ for the $\mathrm{AD}$ group; and $19 \mathrm{~mm}$ for the AC group. The sampling periods are given in millimeter units so to normalize and be capable to apply in other studies.

Furthermore, it was important to set up a decision-making framework in choosing the right software tool in automatic ICV estimation of the four subject groups considered in this study. This was accomplished by evaluating the effectiveness of three widely used software packages (FS, FSL and SPM with their default settings) across PE, PC, AD and AC subject groups. The effectiveness of each software was evaluated from two main perspectives: (1) Determining the accuracy of the automatic tool in measuring ICV as compared with reference manual ICV measurement, and (2) Assessing the reliability and consistency of the results for each of the automated software platforms in measuring ICV across subject groups with these two different neurological conditions.

The versions of the software packages evaluated during the time of the study were the most current; however, SPM 12 was released afterwards. SPM 12, which is reported as a major update for SPM 8, introduced a new built-in utility, "Tissue Volumes," and a manually corrected ICV masks (Malone, Leung et al. 2015) were incorporated into this evaluation of methods. Consequently, SPM 12 ICV estimation with default parameters (Malone, Leung et al. 2015) was performed over the different populations. SPM 12 has substantially improved the ICV estimation for both pediatric control (PC) and adult 
control (AC) groups. SPM 12 also improved the errors in ICV estimation of the PE group, but it introduced a larger systematic bias than SPM 8.

The use of default parameters is not always recommended (Keihaninejad, Heckemann et al. 2010), however, visual inspection of the intermediate processing stages and the manual tuning of the parameters are not often straightforward without having a deep understanding of the software requirements and limitations. Such issues, along with the large number of parameters affecting the end results and the unyielding time requirements of such studies, tend to favor the use of default parameters. To further investigate the aforementioned necessity of adapting the software following a priori knowledge about the population, SPM and FSL software were chosen for their ease in manual intervention.

One potential source of error in ICV estimation across the PE and PC groups could be due to the use of default parameters optimized for the adult population. To evaluate the hypothesis, both SPM 8 and SPM 12 were utilized in conjunction with a pediatric template brain (CCHMC_2_fp) (Wilke, Schmithorst et al. 2002; Wilke and Holland 2003; Wilke, Schmithorst et al. 2003) for spatial normalization purpose to estimate the ICV across the pediatric groups (Table 6.1). Utilization of the pediatric template brain for ICV estimation in SPM 8 across the pediatric groups reduced the systematic bias and errors significantly. The large systematic bias previously reported when using SPM 12 for the PE group was reduced when a pediatric template was used instead. 
Table 6.2: Different versions of SPM software analysis

\begin{tabular}{|c|c|c|c|c|c|c|}
\hline & & PE & & & PC & \\
\hline & $\begin{array}{c}\text { Systematic } \\
\text { Bias }\end{array}$ & $\begin{array}{c}\text { Randomized } \\
\text { Error }\end{array}$ & MRPAD & $\begin{array}{c}\text { Systematic } \\
\text { Bias }\end{array}$ & $\begin{array}{c}\text { Randomized } \\
\text { Error }\end{array}$ & MRPAD \\
\hline SPM 8 & 587.63 & $263.15 \pm 329.54$ & 16.64 & 1081.6 & $339.77 \pm 258.13$ & 20.37 \\
\hline $\begin{array}{l}\text { SPM } \\
\text { 8_PedTemp }\end{array}$ & 618.26 & $179.51 \pm 261.74$ & 11.2 & 592.08 & $355.67 \pm 301.20$ & 21.24 \\
\hline SPM 12 & 1435 & $224.05 \pm 333.10$ & 14.08 & 100.35 & $226.85 \pm 148.27$ & 13.73 \\
\hline \multirow[t]{3}{*}{$\begin{array}{l}\text { SPM } \\
\text { 12_PedTemp }\end{array}$} & -439.12 & $73.38 \pm 122.55$ & 4.65 & -181.57 & $245.36 \pm 238.77$ & 14.50 \\
\hline & \multicolumn{3}{|c|}{ AD } & \multicolumn{3}{|c|}{ AC } \\
\hline & $\begin{array}{c}\text { Systematic } \\
\text { Bias }\end{array}$ & $\begin{array}{c}\text { Randomized } \\
\text { Error }\end{array}$ & MRPAD & $\begin{array}{c}\text { Systematic } \\
\text { Bias }\end{array}$ & $\begin{array}{c}\text { Randomized } \\
\text { Error }\end{array}$ & MRPAD \\
\hline SPM 8 & 644.57 & $189.27 \pm 165.02$ & 12.55 & 37.84 & $151.76 \pm 167.43$ & 10.15 \\
\hline SPM 12 & 1811.6 & $206.5 \pm 312.51$ & 13.39 & -8.89 & $118.14 \pm 120.69$ & 7.95 \\
\hline
\end{tabular}

Systematic Biases and randomized errors are given in $\mathrm{mL}$.

Randomized errors are in the form of Mean $\pm 2 *$ Standard Deviation.

MRPAD: Mean Related Percentage of Absolute Difference; p-value less than 0.01was considered significant.

Finding a self-tuned value for the fractional intensity threshold has been mentioned in studies (Keihaninejad, Heckemann et al. 2010; Piper, Yoong et al. 2014) by performing a hand-tune analysis for a subset of study subjects. Improper selection of this parameter may cause inaccurate estimation of ICV (questionable estimation of FSL of AC population, as shown in Figure 5.5). For example, the fractional intensity threshold (f) parameter in FSL could be changed gradually from 0.1 to 1 with the step of 0.1 and ICV could be estimated across all groups at each value. MRPAD between the estimated ICV for each value and the reference ICV will then be calculated and plotted against the $f$ parameter for each group, as shown in Figure 6. These graphs signify the importance of properly setting the f parameter. Minimum MRPAD were found for each group and the corresponding parameter setting was recorded as the optimized parameter ( 0.1 for PE; 0.2 
for $\mathrm{PC} ; 0.7$ for $\mathrm{AD} ; 0.6$ for $\mathrm{AC})$. From the results obtained in Figure 6.2, it is clear that default value $(0.5)$ is not always the optimized value to be used for the different populations under study. It is observed that in setting up an appropriate value for the $f$ parameter significantly reduced the MRPAD $(p<0.01)$ for the AC and AD groups.

Table 6.3 provides an assessment of MRPAD, systematic bias and randomized error across each population when FSL is used to estimate the ICV with both the default $\mathrm{f}$ parameter and the optimized one. The other important observation to be made on the effect of the threshold selection for estimating ICV in FSL is that the optimized f parameter value (yielding the minimum MRPAD) is less than the default value as provided by FSL $(0.5)$ for the pediatric groups $(0.1$ for PE; 0.2 for PC), and is greater than the default value for the adult groups ( 0.7 for $\mathrm{AD} ; 0.6$ for $\mathrm{AC})$.

Table 6.3: The Effect of $\mathrm{f}$ Parameter self-tuning in using FSL for ICV estimation

\begin{tabular}{|c|c|c|c|c|c|c|}
\hline & \multicolumn{3}{|c|}{$\mathbf{P E}$} & \multicolumn{3}{|c|}{ PC } \\
\hline & $\begin{array}{c}\text { Systematic } \\
\text { Bias }\end{array}$ & $\begin{array}{c}\text { Randomized } \\
\text { Error }\end{array}$ & MRPAD & $\begin{array}{c}\text { Systematic } \\
\text { Bias }\end{array}$ & $\begin{array}{c}\text { Randomized } \\
\text { Error }\end{array}$ & MRPAD \\
\hline $\begin{array}{l}\text { Before } \\
\text { tuning }\end{array}$ & -250.13 & $211.57 \pm 242.63$ & 13.41 & -317.77 & $189.32 \pm 120.18$ & 11.35 \\
\hline \multirow[t]{3}{*}{$\begin{array}{l}\text { After } \\
\text { tuning }\end{array}$} & -146.13 & $140.181 \pm 262.05$ & 8.62 & -111.94 & $151.95 \pm 105.74$ & 9.14 \\
\hline & \multicolumn{3}{|c|}{ AD } & \multicolumn{3}{|c|}{ AC } \\
\hline & $\begin{array}{c}\text { Systematic } \\
\text { Bias }\end{array}$ & $\begin{array}{c}\text { Randomized } \\
\text { Error }\end{array}$ & MRPAD & $\begin{array}{c}\text { Systematic } \\
\text { Bias }\end{array}$ & $\begin{array}{c}\text { Randomized } \\
\text { Error }\end{array}$ & MRPAD \\
\hline $\begin{array}{l}\text { Before } \\
\text { tuning }\end{array}$ & 1312.17 & $704.18 \pm 997.47$ & 91.80 & 1445.31 & $591.79 \pm 790.60$ & 41.74 \\
\hline $\begin{array}{l}\text { After } \\
\text { tuning }\end{array}$ & 1402.68 & $346.73 \pm 214.67$ & 23.60 & 1340.82 & $296.95 \pm 449.68$ & 20.40 \\
\hline
\end{tabular}


The results obtained confirmed the hypothesis that the choice of the software should take into consideration whether the population under study is pediatric or adult, with the knowledge that atlas-based software platforms tend to perform better when dealing with adult populations, unless pediatric templates were used. As a consequence, SPM 12 using pediatric template brain proved to be the most suitable in ICV estimation across the PE group; however, the existence of the relatively large systematic bias needs to be considered.

FSL results after tuning are also fairly accurate across the PE group. SPM12 and FSL (subjected to the tuning step involved) were found to be the most effective tool for ICV estimation across the PC group. FS did not yield accurate results across the pediatric subjects. This may be caused by the assumption that FS considers in which ICV could be estimated reasonably by scaling the ICV of the atlas brain (Buckner 2004). Since the built-in atlas in FS is from adult normal and Alzheimer subjects (Buckner 2004; Heckemann, Keihaninejad et al. 2011) using, the same atlas for ICV measurements in thepediatric population as expected introduce bias.

This is also the reason why FS introduced the least amount of random error across the AD group but it still suffers from a large systematic bias. The minimum systematic bias was observed when using SPM 8. SPM12 and FS are also competing with each other in accurately estimating ICV across AC group; SPM 12 has less systematic bias than FS. On the other hand, SPM-based measurements of ICV showed to be more consistent over different phases of the study across the AC subject group. 

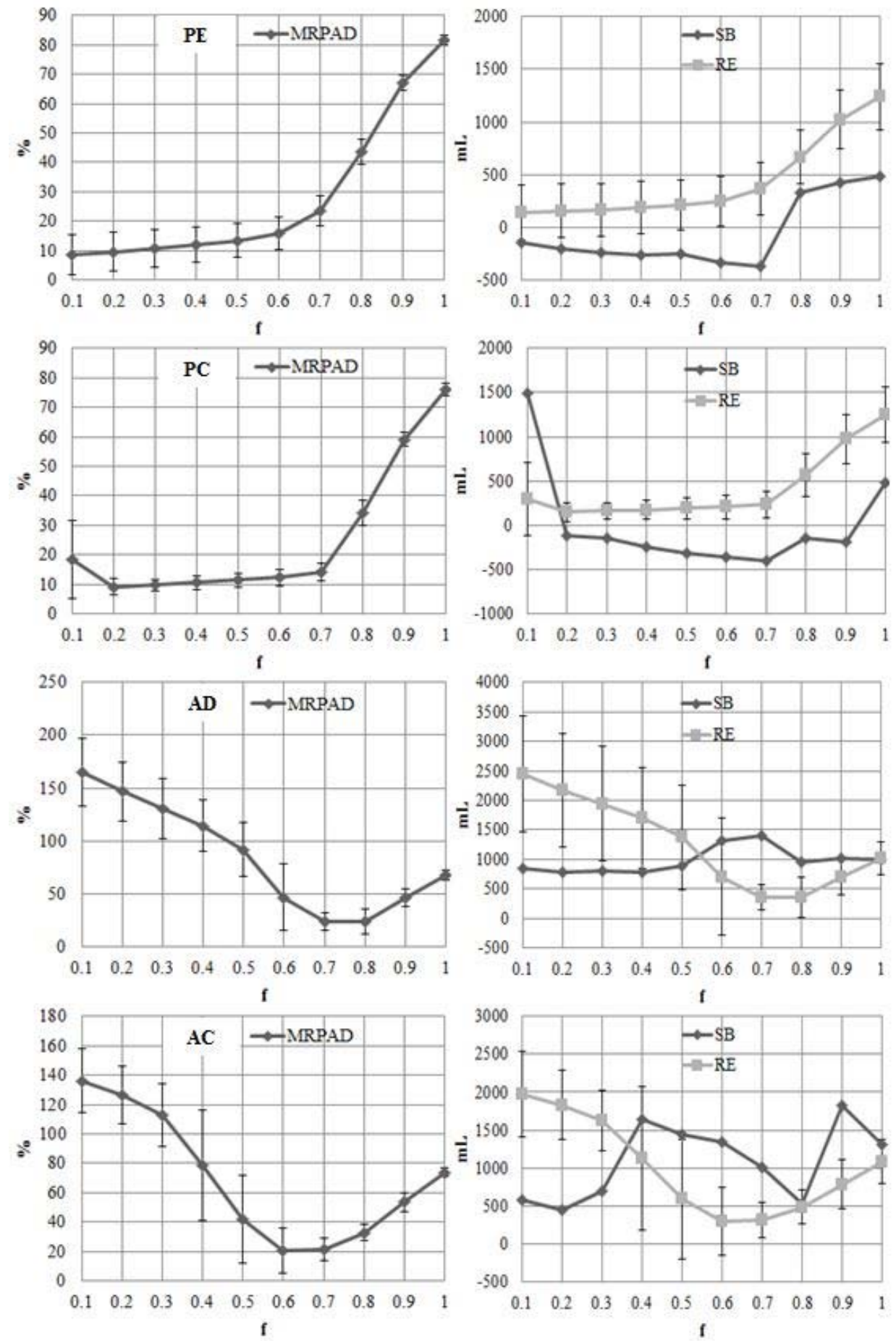

Figure 6.2. Mean Related Percentage Absolute Difference (MRPAD) between reference ICV and estimated ICV using FSL with varying Fractional Intensity Threshold (-f parameter) in the range of 0.1 to 1 with steps of 0.1 across each subject group (PE, PC, $\mathrm{AD}$ and $\mathrm{AC}$ ) (Left) Corresponding plots of errors (SB: Systematic Bias; RE: Randomized Error mean) (Right). 
Another important issue that needs to be taken into account is the necessity of running scan-rescan tests to gauge the variation in ICV estimation. The current study was limited in this sense as no re-scan images were made available by our clinical partners. The importance of scan-rescan test in evaluating segmentation techniques is driven by the fact that the image from one subject could differ if the scan is repeated. In a study by (Morey, Selgrade et al. 2010), the scan-rescan reliability assessment of automatic segmentation of subcortical brain volumes on normal subjects reported substantial variations particularly in small structures such as the amygdala, accumbens, and pallidum.

However, the assumption made here is such variations will be somewhat attenuated when segmenting such a relatively large structure like the intracranial volume; and even if such variation, were to be considered, as this is a comparative study, we believe that such variations in a scan-rescan approach would affect equally the different methods that were evaluated. Other potential biases in automated ICV estimations are also discussed in related studies (Keihaninejad, Heckemann et al. 2010; Nordenskjöld, Malmberg et al. 2013).

\subsection{Recommendations for Future Work}

The generalized construct of the functional connectivity networks could be explored as a function of the neurological disorder and in context to a 3D source, tumour, lesion, or other abnormal physiological or morphological characteristic. Therefore, the research 
work initiated in this dissertation could potentially extend to such tasks as the ones enumerated below:

- Evaluation of the FCN's construction techniques to analyse brain states in other neurological disorders such as ADD/ADHD, Parkinson, and autism.

- Implementation of a graph theory based technique as means to validate the localization of seizure onsets as determined using inverse solutions on the basis of scalp EEG recordings to be compared to subdural recordings when they are available.

- Incorporation of structural information to yield an integrated analysis of functional-structural connectivity networks.

- Extension of the data-driven functional connectivity networks analysis to effective connectivity studies where causality among brain regions could be studied.

- Study of the effect of the segmentation window length in the construction of scalp EEG-based FCN and the corresponding graph theory measures.

- Study of the importance of the different parameters used in automated tools in estimating ICV over different populations, comparing the once deemed relevant to the diagnosis to the ones that are recommended as default parameters.

Brain Functional Connectivity Networks using EEG should be performed while MRI images are acquired simultaneously with EEG. This will help validate the 3D source with any spike-triggered BOLD effect in the MRI together with the pattern of the FCN. Furthermore, retrospective studies on patients that have had EEG and MRI data could be carried out to assess the merits of FCNs in context to computed 3D sources- additional 
data from Baptist Hospital and Miami Children's Hospital will be investigated in the near future 


\section{References}

Adeli, H., Z. Zhou, et al. (2003). "Analysis of EEG records in an epileptic patient using wavelet transform." Journal of neuroscience methods 123(1): 69-87.

Adjouadi, M., M. Cabrerizo, et al. (2005). "An Approach to Functional Brain Mapping using an Inverse Solution Based on the Principal Component Transform." Inverse Problems, Design and Optimization-vol. 2 2: 124.

Adjouadi, M., M. Cabrerizo, et al. (2005). "Detection of interictal spikes and artifactual data through orthogonal transformations." Journal of clinical neurophysiology 22(1): 53-64.

Adjouadi, M., D. Sanchez, et al. (2004). "Interictal spike detection using the Walsh transform." Biomedical Engineering, IEEE Transactions on 51(5): 868-872.

Ahammad, N., T. Fathima, et al. (2014). "Detection of Epileptic Seizure Event and Onset Using EEG." BioMed research international 2014.

Alonso, J. F., M. A. Mañanas, et al. (2006). Connectivity analysis of EEG under drug therapy. Conference proceedings:... Annual International Conference of the IEEE Engineering in Medicine and Biology Society. IEEE Engineering in Medicine and Biology Society. Conference.

Ambarki, K., T. Lindqvist, et al. (2012). "Evaluation of automatic measurement of the intracranial volume based on quantitative MR imaging." American Journal of Neuroradiology 33(10): 1951-1956.

Ansari-Asl, K., L. Senhadji, et al. (2006). "Quantitative evaluation of linear and nonlinear methods characterizing interdependencies between brain signals." Review E 74(3): 031916.

Ashburner, J. and K. J. Friston (2000). "Voxel-based morphometry-the methods." Neuroimage 11(6): 805-821.

Ashburner, J. and K. J. Friston (2005). "Unified segmentation." Neuroimage 26(3): 839851. 
Barkley, G. L. and C. Baumgartner (2003). "MEG and EEG in epilepsy." Journal of clinical neurophysiology 20(3): 163-178.

Bendel, P., T. Koivisto, et al. (2010). "Atrophic enlargement of CSF volume after subarachnoid hemorrhage: correlation with neuropsychological outcome." American Journal of Neuroradiology 31(2): 370-376.

Bounova, G. and O. de Weck (2012). "Overview of metrics and their correlation patterns for multiple-metric topology analysis on heterogeneous graph ensembles." Physical Review E 85(1): 016117.

Buckner, R. L. (2004). "A unified approach for morphometric and functional data analysis in young, old, and demented adults using automated atlas-based head size normalization: reliability and validation against manual measurement of total intracranial volume." Neuroimage 23(2): 724-738.

Cabrerizo, M., M. Adjouadi, et al. (2005). "Integrated study of topographical functional maps based on an auditory comprehension paradigm using an eigensystem study and spectrum analysis." Brain Topography 17(3): 151-163.

Cabrerizo, M., M. Ayala, et al. (2012). "A new parametric feature descriptor for the classification of epileptic and control EEG records in pediatric population." International journal of neural systems 22(02).

Cabrerizo, M., M. Ayala, et al. (2011). "Classification and medical diagnosis of scalp EEG using artificial neural networks." Int J Innovative Computing Information Control 7: 6905-6918.

Cardenas, V. A., L. L. Chao, et al. (2005). "Using automated morphometry to detect associations between ERP latency and structural brain MRI in normal adults." Human brain mapping 25(3): 317-327.

Chee, M. W. L., H. Zheng, et al. (2011). "Brain structure in young and old East Asians and Westerners: comparisons of structural volume and cortical thickness." Journal of Cognitive Neuroscience 23(5): 1065-1079.

Control, C. f. D. and Prevention (2012). "Epilepsy in adults and access to care--United States, 2010." MMWR. Morbidity and mortality weekly report 61(45): 909. 
Cox, R. W. (1996). "AFNI: software for analysis and visualization of functional magnetic resonance neuroimages." Computers and Biomedical research 29(3): 162-173.

Cullen, K. R., S. Wallace, et al. (2012). "Cigarette smoking and white matter microstructure in schizophrenia." Psychiatry Research: Neuroimaging 201(2): 152-158.

Dale, A. M., B. Fischl, et al. (1999). "Cortical surface-based analysis: I. Segmentation and surface reconstruction." Neuroimage 9(2): 179-194.

Dhillon, I. S., P. K. Ravikumar, et al. (2011). Nearest neighbor based greedy coordinate descent. Advances in Neural Information Processing Systems.

Douw, L., M. De Groot, et al. (2010). "'Functional connectivity'is a sensitive predictor of epilepsy diagnosis after the first seizure." PloS one 5(5): e10839.

Douw, L., E. van Dellen, et al. (2010). "Epilepsy is related to theta band brain connectivity and network topology in brain tumor patients." BMC neuroscience 11(1): 103 .

Duara, R., D. A. Loewenstein, et al. (2010). "Reliability and validity of an algorithm for the diagnosis of normal cognition, mild cognitive impairment, and dementia: implications for multicenter research studies." The American Journal of Geriatric Psychiatry 18(4): 363-370.

Dukart, J., K. Mueller, et al. (2013). "Relationship between Imaging Biomarkers, Age, Progression and Symptom Severity in Alzheimer's Disease." NeuroImage: Clinical.

Eddin, A. S., J. Wang, et al. (2013). ICA-based connectivity on brain networks using fMRI. Neural Engineering (NER), 2013 6th International IEEE/EMBS Conference on, IEEE.

Eddin, A. S., J. Wang, et al. (2014). "The effects of pediatric epilepsy on a language connectome." Human brain mapping. 
Ehrlich, S., E. M. Morrow, et al. (2010). "The COMT Val108/158Met polymorphism and medial temporal lobe volumetry in patients with schizophrenia and healthy adults." Neuroimage 53(3): 992-1000.

England, M. J., C. T. Liverman, et al. (2012). "Epilepsy across the spectrum: Promoting health and understanding.: A summary of the Institute of Medicine report." Epilepsy \& Behavior 25(2): 266-276.

Eritaia, J., S. J. Wood, et al. (2000). "An optimized method for estimating intracranial volume from magnetic resonance images." Magnetic Resonance in Medicine 44(6): 973-977.

Fennema-Notestine, C., D. J. Hagler, et al. (2009). "Structural MRI biomarkers for preclinical and mild Alzheimer's disease." Human brain mapping 30(10): 32383253 .

Folstein, M. F., S. E. Folstein, et al. (1975). "“Mini-mental state": a practical method for grading the cognitive state of patients for the clinician." Journal of psychiatric research 12(3): 189-198.

Friedman, J., T. Hastie, et al. (2010). "Regularization paths for generalized linear models via coordinate descent." Journal of statistical software 33(1): 1.

Friston, K. J. (1994). "Functional and effective connectivity in neuroimaging: a synthesis." Human brain mapping 2(1-2): 56-78.

Garibotto, V., B. Borroni, et al. (2011). "Subcortical and deep cortical atrophy in frontotemporal lobar degeneration." Neurobiology of aging 32(5): 875-884.

Germeyan, S. C., D. Kalikhman, et al. (2014). "Automated versus manual hippocampal segmentation in preoperative and postoperative patients with epilepsy." Epilepsia.

Ghosh, A., Y. Rho, et al. (2008). "Cortical network dynamics with time delays reveals functional connectivity in the resting brain." Cognitive neurodynamics 2(2): 115120. 
Gillies, C., N. Patel, et al. (2012). "Gene expression classification using binary rule majority voting genetic programming classifier." International Journal of Advanced Intelligence Paradigms 4(3): 241-255.

Gillies, C. E., X. Gao, et al. (2012). Improved Feature Selection by Incorporating Gene Similarity into the LASSO. Data Mining Workshops (ICDMW), 2012 IEEE 12th International Conference on, IEEE.

Gong, G., R. P. Alexander, et al. (2012). "Isolated febrile seizures are not associated with structural abnormalities of the limbic system." Epilepsy research.

Graca, A. M., K. R. V. Cardoso, et al. (2013). "Cerebral volume at term age: Comparison between preterm and term-born infants using cranial ultrasound." Early human development.

Groves, A. R., S. M. Smith, et al. (2012). "Benefits of multi-modal fusion analysis on a large-scale dataset: life-span patterns of inter-subject variability in cortical morphometry and white matter microstructure." Neuroimage.

Guillen, M. R., M. Adjouadi, et al. (2009). A knowledge-based database system for visual rating of fMRI activation patterns for brain language networks. The Fifth Richard Tapia Celebration of Diversity in Computing Conference: Intellect, Initiatives, Insight, and Innovations, ACM.

Guo, L., D. Rivero, et al. (2010). "Epileptic seizure detection using multiwavelet transform based approximate entropy and artificial neural networks." Journal of neuroscience methods 193(1): 156-163.

Han, J. and M. Kamber (2006). Data Mining, Southeast Asia Edition: Concepts and Techniques, Morgan kaufmann.

Heckemann, R. A., S. Keihaninejad, et al. (2011). "Automatic morphometry in Alzheimer's disease and mild cognitive impairment." Neuroimage 56(4): 20242037.

Huang, A. (2008). Similarity measures for text document clustering. Proceedings of the sixth new zealand computer science research student conference (NZCSRSC2008), Christchurch, New Zealand. 
Ikram, M. A., M. Fornage, et al. (2012). "Common variants at 6q22 and 17q21 are associated with intracranial volume." Nature genetics 44(5): 539.

Jack, C. R., D. S. Knopman, et al. (2012). "An operational approach to National Institute on Aging-Alzheimer's Association criteria for preclinical Alzheimer disease." Annals of neurology 71(6): 765-775.

Jack Jr, C. R., D. S. Knopman, et al. (2013). "Tracking pathophysiological processes in Alzheimer's disease: an updated hypothetical model of dynamic biomarkers." The Lancet Neurology 12(2): 207-216.

Jenkinson, M., C. F. Beckmann, et al. (2012). "Fsl." Neuroimage 62(2): 782-790.

Jenkinson, M., M. Pechaud, et al. (2005). BET2: MR-based estimation of brain, skull and scalp surfaces. Eleventh annual meeting of the organization for human brain mapping.

Jirsa, V. K. (2004). "Connectivity and dynamics of neural information processing." Neuroinformatics 2(2): 183-204.

Jung, T.-P., S. Makeig, et al. (2000). "Removing electroencephalographic artifacts by blind source separation." Psychophysiology 37(02): 163-178.

Kaiser, J. F. (1990). On a simple algorithm to calculate theenergy'of a signal, Acoustics, Speech, and Signal Processing, 1990. ICASSP-90., 1990 International Conference on.

Keihaninejad, S., R. A. Heckemann, et al. (2010). "A robust method to estimate the intracranial volume across MRI field strengths (1.5 T and 3T)." Neuroimage 50(4): 1427-1437.

Kim, S.-H., C. Faloutsos, et al. (2013). "Coercively Adjusted Auto Regression Model for Forecasting in Epilepsy EEG." Computational and mathematical methods in medicine 2013.

Kirmani, B. F. (2013). "Importance of Video-EEG Monitoring in the Diagnosis of Epilepsy in a Psychiatric Patient." Case reports in neurological medicine 2013. 
Kochan, N. A., M. Breakspear, et al. (2011). "Cortical responses to a graded working memory challenge predict functional decline in mild cognitive impairment." Biological psychiatry 70(2): 123-130.

Kohavi, R. and G. H. John (1997). "Wrappers for feature subset selection." Artificial intelligence 97(1): 273-324.

Konganti, K., G. Wang, et al. (2013). "SBEToolbox: a Matlab toolbox for biological network analysis." Evolutionary bioinformatics online 9: 355.

Lagerlund, T. D., F. W. Sharbrough, et al. (1997). "Spatial filtering of multichannel electroencephalographic recordings through principal component analysis by singular value decomposition." Journal of clinical neurophysiology 14(1): 73-82.

Lahlou, M., M. Guillen, et al. (2006). An online web-based repository site of fMRI medical images and clinical data for childhood epilepsy. 11th world congress on internet in medicine.

Lampert, E. J., K. R. Choudhury, et al. (2013). "Prevalence of Alzheimer's Pathologic Endophenotypes in Asymptomatic and Mildly Impaired First-Degree Relatives." PloS one 8(4): e60747.

Lang, E. W., A. Tomé, et al. (2012). "Brain connectivity analysis: a short survey." Computational intelligence and neuroscience 2012: 8.

Lee, U., S. Kim, et al. (2006). "Classification of epilepsy types through global network analysis of scalp electroencephalograms." Physical Review E 73(4): 041920.

Lowe, M., B. Mock, et al. (1998). "Functional connectivity in single and multislice echoplanar imaging using resting-state fluctuations." Neuroimage 7(2): 119-132.

Malone, I. B., K. K. Leung, et al. (2015). "Accurate automatic estimation of total intracranial volume: A nuisance variable with less nuisance." Neuroimage 104: 366-372.

Martin Bland, J. and D. Altman (1986). "Statistical methods for assessing agreement between two methods of clinical measurement." The Lancet 327(8476): 307-310. 
McCullagh, P., J. A. Nelder, et al. (1989). Generalized linear models, Chapman and Hall London.

Morey, R. A., E. S. Selgrade, et al. (2010). "Scan-rescan reliability of subcortical brain volumes derived from automated segmentation." Human brain mapping 31(11): 1751-1762.

Mormann, F., T. Kreuz, et al. (2005). "On the predictability of epileptic seizures." Clinical neurophysiology 116(3): 569-587.

Nordenskjöld, R. (2014). "Analysis of Human Brain MRI: Contributions to Regional Volume Studies."

Nordenskjöld, R., F. Malmberg, et al. (2013). "Intracranial volume estimated with commonly used methods could introduce bias in studies including brain volume measurements." Neuroimage.

Ogawa, S., T.-M. Lee, et al. (1990). "Brain magnetic resonance imaging with contrast dependent on blood oxygenation." Proceedings of the National Academy of Sciences 87(24): 9868-9872.

Oostenveld, R., P. Fries, et al. (2011). "FieldTrip: open source software for advanced analysis of MEG, EEG, and invasive electrophysiological data." Computational intelligence and neuroscience 2011: 1 .

Pa, J., A. Boxer, et al. (2009). "Clinical-neuroimaging characteristics of dysexecutive mild cognitive impairment." Annals of neurology 65(4): 414-423.

Pengas, G., J. Pereira, et al. (2009). "Comparative reliability of total intracranial volume estimation methods and the influence of atrophy in a longitudinal semantic dementia cohort." Journal of Neuroimaging 19(1): 37-46.

Peper, J. S., H. G. Schnack, et al. (2009). "Heritability of regional and global brain structure at the onset of puberty: A magnetic resonance imaging study in 9-yearold twin pairs." Human brain mapping 30(7): 2184-2196.

Pereda, E., R. Q. Quiroga, et al. (2005). "Nonlinear multivariate analysis of neurophysiological signals." Progress in neurobiology 77(1): 1-37. 
Piguet, O., Å. Petersén, et al. (2011). "Eating and hypothalamus changes in behavioralvariant frontotemporal dementia." Annals of neurology 69(2): 312-319.

Pillai, J. and M. R. Sperling (2006). "Interictal EEG and the diagnosis of epilepsy." Epilepsia 47(s1): 14-22.

Piper, R. J., M. M. Yoong, et al. (2014). "Estimating intracranial volume using intracranial area in healthy children and those with childhood status epilepticus." Brain and behavior 4(6): 936-942.

Ponten, S., F. Bartolomei, et al. (2007). "Small-world networks and epilepsy: graph theoretical analysis of intracerebrally recorded mesial temporal lobe seizures." Clinical neurophysiology 118(4): 918-927.

Ponten, S., L. Douw, et al. (2009). "Indications for network regularization during absence seizures: weighted and unweighted graph theoretical analyses." Experimental neurology 217(1): 197-204.

Quraan, M. A., C. McCormick, et al. (2013). "Altered Resting State Brain Dynamics in Temporal Lobe Epilepsy Can Be Observed in Spectral Power, Functional Connectivity and Graph Theory Metrics." PloS one 8(7): e68609.

Reynolds, D. (2009). "Gaussian mixture models." Encyclopedia of Biometrics: 659-663.

Ridgway, G., J. Barnes, et al. (2011). "Estimation of total intracranial volume; a comparison of methods." Alzheimer's and Dementia 7(4): S62-S63.

Ritvanen, A. G., M. E. de Oliveira, et al. (2013). "Mesh-based method for measuring intracranial volume in patients with craniosynostosis." International journal of computer assisted radiology and surgery: 1-7.

Roussotte, F. F., K. K. Sulik, et al. (2012). "Regional brain volume reductions relate to facial dysmorphology and neurocognitive function in fetal alcohol spectrum disorders." Human brain mapping 33(4): 920-937.

Rubinov, M. and O. Sporns (2010). "Complex network measures of brain connectivity: uses and interpretations." Neuroimage 52(3): 1059-1069. 
Salah Eddin, A. (2013). "Network Construction and Graph Theoretical Analysis of Functional Language Networks in Pediatric Epilepsy."

Sargolzaei, A., K. Faez, et al. (2009). A new robust wavelet based algorithm for baseline wandering cancellation in ECG signals. Signal and Image Processing Applications (ICSIPA), 2009 IEEE International Conference on, IEEE.

Sargolzaei, A., K. Faez, et al. (2011). A new method for Foetal Electrocardiogram extraction using Adaptive Nero-Fuzzy Interference System trained with PSO algorithm. Electro/Information Technology (EIT), 2011 IEEE International Conference on, IEEE.

Sargolzaei, A., K. K. Yen, et al. (2013). "Time-Delay Switch Attack on Load Frequency Control in Smart Grid)." Advances in Communication Technology 5: 55-64.

Sargolzaei, S. (2012). "Impact of Accelerometry and Spirography Data Analysis of Essential Tremor on BOLD-fMRI Data Interpretation."

Sargolzaei, S., M. Cabrerizo, et al. "Scalp EEG brain functional connectivity networks in pediatric epilepsy." Computers in biology and medicine $(0)$.

Sargolzaei, S., M. Cabrerizo, et al. (2013). Functional connectivity network based on graph analysis of scalp EEG for epileptic classification. Signal Processing in Medicine and Biology Symposium (SPMB), 2013 IEEE, IEEE.

Sargolzaei, S., A. S. Eddin, et al. (2013). Resting state functional connectivity based on principal component transformation of cortical fMRI measurements. Neural Engineering (NER), 2013 6th International IEEE/EMBS Conference on, IEEE.

Sargolzaei, S., K. Faez, et al. (2008). Signal processing based for fetal electrocardiogram extraction. BioMedical Engineering and Informatics, 2008. BMEI 2008. International Conference on, IEEE.

Sargolzaei, S., M. Goryawala, et al. (2014). Comparative reliability analysis of publicly available software packages for automatic intracranial volume estimation. Engineering in Medicine and Biology Society (EMBC), 2014 36th Annual International Conference of the IEEE, IEEE. 
Schoffelen, J. M. and J. Gross (2009). "Source connectivity analysis with MEG and EEG." Human brain mapping 30(6): 1857-1865.

Seth, A. K. (2010). "A MATLAB toolbox for Granger causal connectivity analysis." Journal of neuroscience methods 186(2): 262-273.

Smit, D. J., C. J. Stam, et al. (2008). "Heritability of "small-world" networks in the brain: A graph theoretical analysis of resting-state EEG functional connectivity." Human brain mapping 29(12): 1368-1378.

Sonmez, O. F., Y. Temel, et al. (2013). "A new evaluation method for the intracranial volume changes and subdural effusion of patients following endoscopic third ventriculostomy." Clinical neurology and neurosurgery 115(2): 160-164.

Subasi, A., A. Alkan, et al. (2005). "Wavelet neural network classification of EEG signals by using AR model with MLE preprocessing." Neural Networks 18(7): 985-997.

Subasi, A. and E. Erçelebi (2005). "Classification of EEG signals using neural network and logistic regression." Computer Methods and Programs in Biomedicine 78(2): 87-99.

Szentkuti, A., S. Guderian, et al. (2004). "Quantitative MR analyses of the hippocampus: unspecific metabolic changes in aging." Journal of neurology 251(11): 13451353.

Taki, Y., B. Thyreau, et al. (2013). "A longitudinal study of the relationship between personality traits and the annual rate of volume changes in regional gray matter in healthy adults." Human brain mapping 34(12): 3347-3353.

Thambisetty, M., Y. An, et al. (2012). "Plasma clusterin concentration is associated with longitudinal brain atrophy in mild cognitive impairment." Neuroimage 59(1): 212-217.

Towle, V. L., J. Bolaños, et al. (1993). "The spatial location of EEG electrodes: locating the best-fitting sphere relative to cortical anatomy." Electroencephalography and clinical neurophysiology 86(1): 1-6. 
Trivedi, M. A., T. R. Stoub, et al. (2011). "Entorhinal cortex volume is associated with episodic memory related brain activation in normal aging and amnesic mild cognitive impairment." Brain Imaging and Behavior 5(2): 126-136.

Van Dellen, E., L. Douw, et al. (2009). "Long-term effects of temporal lobe epilepsy on local neural networks: a graph theoretical analysis of corticography recordings." PloS one 4(11): e8081.

Van Durme, B. and A. Lall (2010). Online generation of locality sensitive hash signatures. Proceedings of the ACL 2010 Conference Short Papers, Association for Computational Linguistics.

Van Wijk, B. C., C. J. Stam, et al. (2010). "Comparing brain networks of different size and connectivity density using graph theory." PloS one 5(10): e13701.

Wang, Z., Z. Zhang, et al. (2012). "Impairments of thalamic nuclei in idiopathic generalized epilepsy revealed by a study combining morphological and functional connectivity MRI." PloS one 7(7): e39701.

Weisberg, S. (2005). Applied linear regression, John Wiley \& Sons.

Westman, E., C. Aguilar, et al. (2013). "Regional magnetic resonance imaging measures for multivariate analysis in Alzheimer's disease and mild cognitive impairment." Brain Topography 26(1): 9-23.

Whitwell, J. L., W. R. Crum, et al. (2001). "Normalization of cerebral volumes by use of intracranial volume: implications for longitudinal quantitative MR imaging." American Journal of Neuroradiology 22(8): 1483-1489.

Wilke, C., G. Worrell, et al. (2011). "Graph analysis of epileptogenic networks in human partial epilepsy." Epilepsia 52(1): 84-93.

Wilke, M. and S. K. Holland (2003). "Variability of gray and white matter during normal development: a voxel-based MRI analysis." Neuroreport 14(15): 1887.

Wilke, M., V. Schmithorst, et al. (2003). "Normative pediatric brain data for spatial normalization and segmentation differs from standard adult data." Magnetic Resonance in Medicine 50(4): 749-757. 
Wilke, M., V. J. Schmithorst, et al. (2002). "Assessment of spatial normalization of whole-brain magnetic resonance images in children." Human brain mapping 17(1): 48-60.

Winston, G. P., M. J. Cardoso, et al. (2013). "Automated hippocampal segmentation in patients with epilepsy: Available free online." Epilepsia 54(12): 2166-2173.

Wu, L. and J. Gotman (1998). "Segmentation and classification of EEG during epileptic seizures." Electroencephalography and clinical neurophysiology 106(4): 344-356.

Xia, M., J. Wang, et al. (2013). "BrainNet Viewer: a network visualization tool for human brain connectomics." PloS one 8(7): e68910.

Xie, S. and S. Krishnan (2014). "Dynamic Principal Component Analysis with Nonoverlapping Moving Window and Its Applications to Epileptic EEG Classification." The Scientific World Journal 2014.

Xu, J.-W., H. Bakardjian, et al. (2007). A new nonlinear similarity measure for multichannel biological signals. Neural Networks, 2007. IJCNN 2007. International Joint Conference on, IEEE.

Zhou, Q., M. Goryawala, et al. (2014). "Significance of Normalization on Anatomical MRI Measures in Predicting Alzheimer's Disease." The Scientific World Journal 2014.

Ziaei, A., A. Sangwan, et al. (2012). Prof-Life-Log: Audio Environment Detection for Naturalistic Audio Streams. INTERSPEECH.

Ziaei, A., H. Yeganeh, et al. (2008). A novel approach for contrast enhancement in biomedical images based on histogram equalization. BioMedical Engineering and Informatics, 2008. BMEI 2008. International Conference on, IEEE.

Zwoliński, P., M. Roszkowski, et al. (2010). "Open database of epileptic EEG with MRI and postoperational assessment of foci-a real world verification for the EEG inverse solutions." Neuroinformatics 8(4): 285-299. 
VITA

\section{SAMAN SARGOLZAEI}

2006

2009

2012

2015
B.S., Electrical and Computer Engineering Mazandaran University of Sciences and Technology Babol, Iran

M.S., Electrical Engineering Amirkabir University of Technology (Tehran Polytechnic)

Tehran, Iran

M.S., Electrical Engineering University of Miami

Miami, Florida

Ph.D. Candidate, Electrical Engineering

Florida International University

Miami, Florida

World's Ahead Graduate (Spring 2015), Florida International University, April 2015.

Outstanding Student Life Award, Graduate Student Leadership Award, Division of Studenat Affairs, Florida International University, April 2015.

SGA Graduate Scholarship Recipient, Florida International University, January 2015.

Charles E. Perry (FIU's founding president) Graduate Scholarship Recipient, Florida International University, September 2014.

Dean's Merit and Leadership Scholarship Recipient, College of Engineering and Computing, Florida International University, July 2014.

$1^{\text {st }}$ place award in Engineering category poster presentation competition, GSAW 2014 Scholarly forum, March 2014.

\section{PUBLICATIONS}

S. Sargolzaei, A. Sargolzaei, M. Cabrerizo, M. Goryawala, G. Chen, A. Pinzon-Ardila, S. M. Gonzalez-Arias and M. Adjouadi, "Estimating Intracranial Volume (ICV) in brain research: guideline for an informed decision-making process," Neuroinformatics, 10.1007/s12021-015-9266-5, 2015. 
S. Sargolzaei, A. Sargolzaei, M. Cabrerizo, M. Goryawala, Q. Zhou, S. Noei, G. Chen, R. Duara, W. Barker and M. Adjouadi, "Intracranial volume estimation in patients with Alzheimer's disease,” BMC Bioinformatics, Vol. 16, Suppl. 7, 2015.

S. Sargolzaei, M. Cabrerizo, A. Sargolzaei, S. Noei, H. Rajaei, A. Salah Eddin, A. Pinzon-Ardila, S. M. Gonzalez Arias, P. Jayakar and M. Adjouadi, "A probabilistic approach for pediatric epilepsy diagnosis using brain functional connectivity networks," Accepted for publication in BMC Bioinformatics, Vol. 16, Suppl. 7, 2015.

S. Sargolzaei, M. Cabrerizo, M. Goryawala, A. Salah Eddin and M. Adjouadi, "Scalp EEG brain functional connectivity networks in pediatric epilepsy," Computers in Biology and Medicine, vol. 56; pp. 56C:158-166, 2015.

A. Salah Eddin, J. Wang, W. Wu, S. Sargolzaei, B. Bjornson, R. A. Jones, W. D. Gaillard and M. Adjouadi, "The effects of pediatric epilepsy on a language connectome," Human brain mapping, Vol. 35, no. 12, pp. 5996-6010, 2014.

S. Sargolzaei, A. Sargolzaei, M. Cabrerizo, M. Goryawala, Q. Zhou, S. Noei, G. Chen, R. Duara, W. Barker and M. Adjouadi, "Practical considerations for estimating intracranial volume in patients with Alzheimer's disease," CD Proc. Biotechnology and Bioinformatics Symposium BIOT 2014, December 2014.

S. Sargolzaei, M. Cabrerizo, A. Sargolzaei, S. Noei, H. Rajaei, A. Salah Eddin, A. Pinzon-Ardila, S. M. Gonzalez-Arias, P. Jayakar and M. Adjouadi, "Gaussian mixture model for pediatric epilepsy diagnosis using brain functional connectivity networks," CD Proc. Biotechnology and Bioinformatics Symposium BIOT 2014, December 2014.

S. Sargolzaei, M. Goryawala, M. Cabrerizo, G. Chen, P. Jayakar, R. Duara, W. Barker and M. Adjouadi, "Comparative reliability analysis of publicly available software packages for automatic intracranial volume estimation," Proc. IEEE $36^{\text {th }}$ Eng. In Med. and Biol. Conf., pp. 2342-2345, August 2014.

S. Sargolzaei, M. Cabrerizo, M. Goryawala, A. Salah Eddin and M. Adjouadi, "Functional connectivity network based on graph analysis of scalp EEG for epileptic classification," Proc. IEEE Signal proc. in Med. and Biol. Conf., pp. 1-4, December 2013.

S. Sargolzaei, A. Salah Eddin, M. Cabrerizo and M. Adjouadi, "Resting state functional connectivity based on principal component transformation of cortical fMRI measurements," Proc. IEEE/EMBS $6^{\text {th }}$ international Neural Eng., pp. 1501-1504, November 2013. 